\title{
CREATING A BUZZ
}

Maddison Cooper

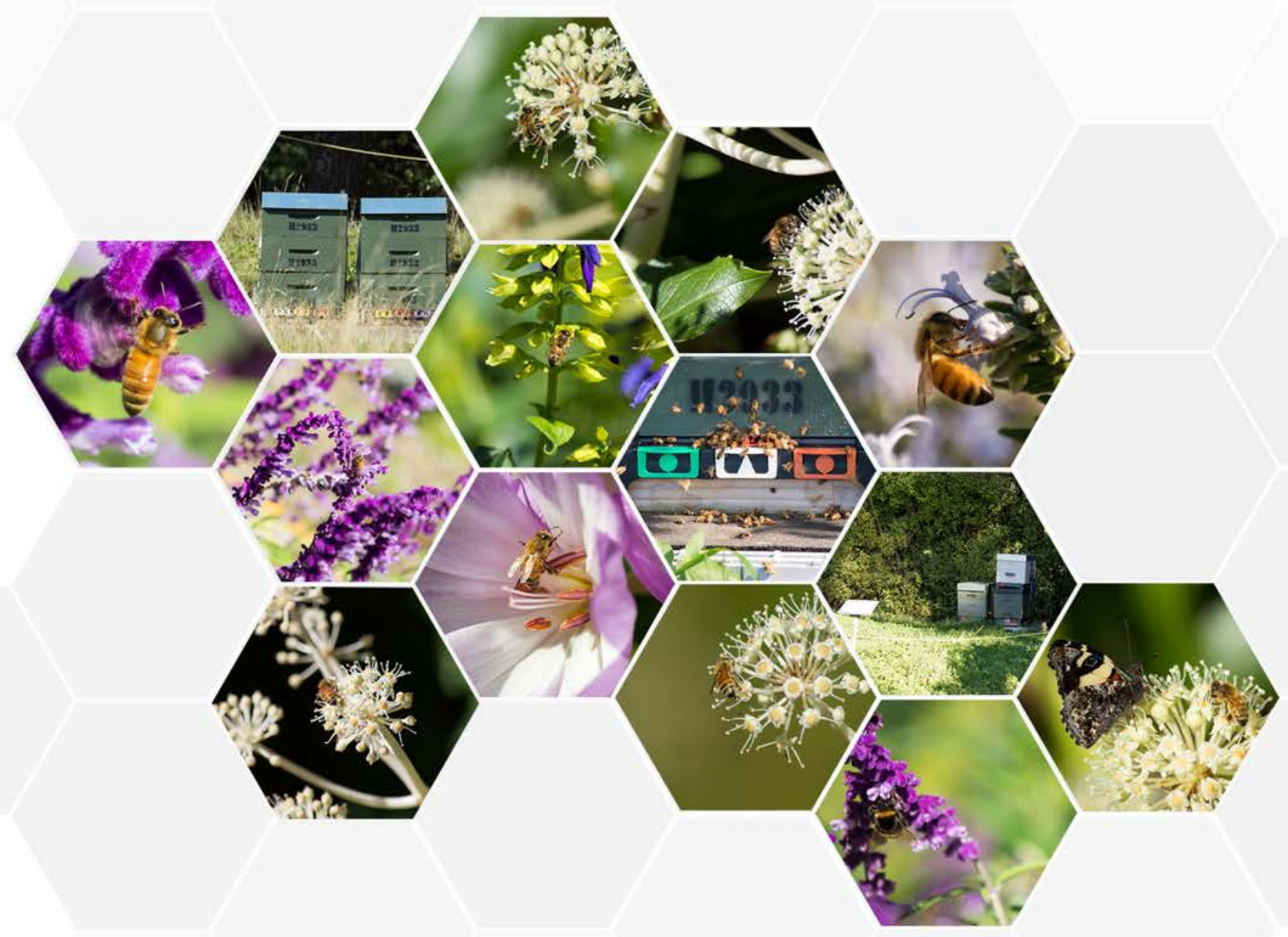

A thesis submitted to the Victoria University of Wellington in fulfilment of the requirements for the degree of Master of Design Innovation

Victoria University of Wellington 2017 


\section{Acknowledgements}

This thesis would not have been possible without the guidance and encouragement of my supervisors Tonya Sweet and Dr Catherine Caudwell. I am especially indebted to them for their continuous hard work whilst making me realise my full potential and providing me with the best academic support.

I am extremely grateful to all of those whom I have had the pleasure of meeting and talking to during this process. Your expertise, interests and attitudes have motivated and inspired me throughout my work and have shaped the outcome of this thesis. I would like to especially thank members from Victoria University of Wellington, Wellington Beekeepers Association, Wellington Botanic Gardens and the Beeple Honey Collective.

Nobody has been more important to me in the pursuit of this thesis than the members of my family. I would like to thank my ultimate role models, my parents, whose love and guidance is with me in whatever I pursue. Most importantly, I would like to dedicate this thesis to my late Grandad Trev, who initially inspired this research idea. 


\title{
1 Abstract
}

\author{
How might communication design be utilised to raise awareness about the \\ sustained well-being of Wellington City's honey bee populations?
}

Human concern and intervention has been pivotal in maintaining the well-being of bee populations in Wellington City. Through the applied knowledge of bee keepers, the health of local beehives has been sustained. This status, however, is increasingly challenged. Without human interactions, bee colonies are now unable to survive due to a number of external interferences, including climate change and the growing urbanisation of the natural habitat. Within this context, support for the care-taking practices of our bees requires prioritisation. Supported by Wellington's inclusion in the 100 Resilient Cities (2017) initiative pioneered by the Rockefeller Foundation, the cultivation of resilience in regards to contemporary physical, social and economic challenges is imperative in the 21st Century. The motivation to protect Wellington's honey bee populations reflects global concerns about declining bee populations that are the result of changing ecological impacts. Through the development of communication platforms that function to raise awareness and appreciation about the important role of bees in sustaining Wellington's native flora, honey production, and environment at large, this valuable natural resource and cohabitant will ideally see increased support. With the considered application of communication design, the importance of Wellington's urban honey bee populations can be reinforced and existing knowledge can be shared throughout the community, including the next generation of bee keepers.

Currently, no quantitative information exists that enables an insight into the perception of relationships with bees as cohabitants alongside human populations and environments, and there is little in the way of campaigns that promote positive interactions with the bees that live amongst us. Positive relationships with nature are needed to enhance resilience, and to confront, and change the negative climatic influences. Regardless of whether these challenges are man-made or naturally occurring, Wellington City has the potential to embrace positive and sustainable transformation and prosperity. A literature review pursued as part of this research revealed three key themes: sustainable environments; honey bee populations; and the potential value of communication design to promote awareness and to invite action. Quantitative research addresses a professional perspective and this is pursued through semistructured interviews with experts who have a stake in the well-being of Wellington's honey bee industries.

The objective of this research entails the application of communication design in the development of a campaign that has the ability to communicate a movement across multiple platforms, provoking positive environmental influences and behaviours amongst Wellington City's young adult population. This research aims to connect passionate individuals in communities who have an interest in supporting the sustainability of our local honey bee ecologies with new-comers who are open to learning sustainable bee practices. Through providing a holistic presentation of current practices and bee populations within Wellington, and information regarding the potential threats these populations face, this campaign will enable this generation of Wellingtonians to be prepared to protect this vital species. This research aims to examine ways that communication design might be utilised through different platforms to raise awareness of the value local honey bees have within our environments. The campaign goal is to include educational tools that interact with Wellington residents, specifically young adults, to encourage a sustained support towards honey bee well-being. 


\section{Table of Contents}

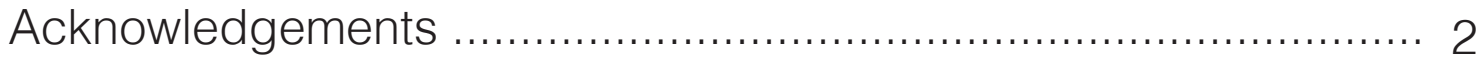

ONE $3-5$

Abstract 3

Contents Page .......................................................... 4

TWO List of Figures ...................................................... 5

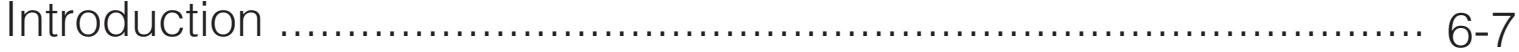

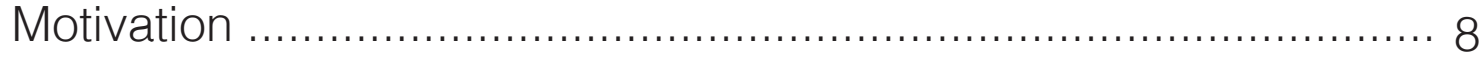

THREE 11-31

Literature \& Design Review ........................................... 11-31

Literature Review .................................................. 11-21

Design Precedents .................................................... 23-31

FOUR

Research Methodology ................................................ 33-39

FIVE

Communication Design Principles ................................ 37-38

Stakeholder Perspectives \& Design Recommendations .............. 41-47

SIX $49-72$

Design Process 49-89

Communication Design Criteria .................................... 50

Website Design Criteria .................................................. 51

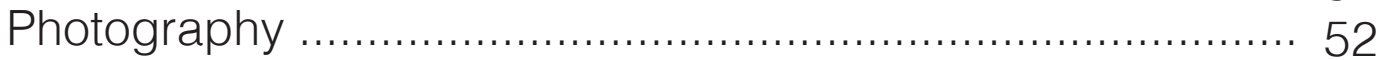

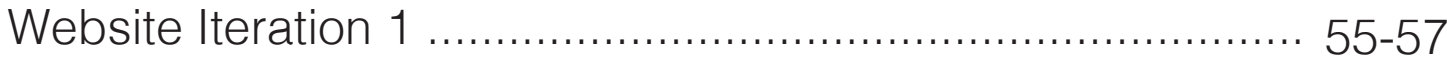

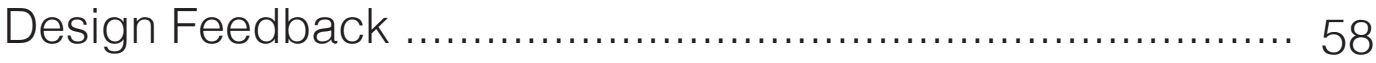

Website Iteration 2 ................................................ 59-62

Design Feedback ......................................................... 63

Website Iteration 3 ............................................... 64-69

Physical Locations ........................................................ $70-71$

SEVEN

Social Media Outputs .............................................. 72

Final Design Prototype ..................1. 75-89

EIGHT $\quad 91-94$

Conclusion .............................................................. 91-94

Summary of Process \& Chapters .................................... 93

Future Pathways ...................................................... 93

Personal Reflection ...................................................... 94

NINE

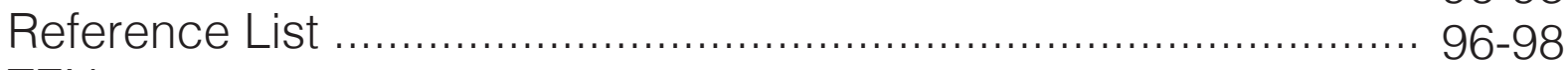

TEN $\quad 99$

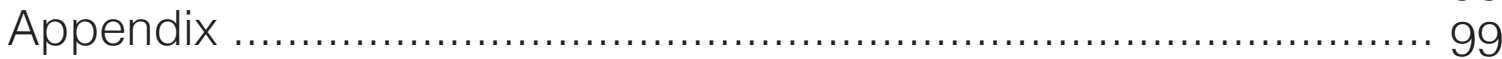

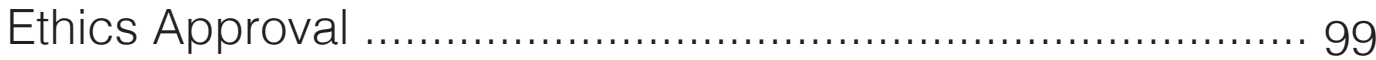




\section{List of Figures}

\section{Precedents}

\section{Page 24}

Figure 4.1. Smuts-Kennedy, S., Tairoa, T. (2014). Park Pop Project 02 (Art Installation) [Digital Photograph]. Retrieved from http://www.pop.org.nz/projects/thepark/

Figure 4.2. Smuts-Kennedy, S., Tairoa, T. (2014). The Park (Art Installation) [Digital Photograph]. Retrieved from http://www.pop.org.nz/projects/thepark/

Figure 4.3. Smuts-Kennedy, S., Tairoa, T. (2014). Victoria Park Meadow (Art Installation) [Digital Photograph]. Retrieved from http://www.pop.org.nz/projects/thepark/

\section{Page 26}

Figure 5.1. Faccin, F. (2015). Micro Architecture for Bees (Public Installation) [Digital Photograph]. Retrieved from https://www. dezeen.com/2015/07/01/francesco-faccin-honey-factory-urbanbeehive-bees-triennale-museum-milan-expo-2015/

Figure 5.2. Faccin, F. (2015). Beekeeper \& Beehive (Public Installation) [Digital Photograph]. Retrieved from https://www. dezeen.com/2015/07/01/francesco-faccin-honey-factory-urbanbeehive-bees-triennale-museum-milan-expo-2015/

Figure 5.3. Faccin, F. (2015). Built Structure (Public Installation) [Digital Photograph]. Retrieved from https://www.dezeen.com/2015/07/01/francesco-faccin-honey-factory-urban-beehive-beestriennale-museum-milan-expo-2015/

\section{Page 28}

Figure 6.1. Buttress, W. (2017). "The Hive" Royal Botanic Gardens, Kew, London (Art Installation) [Digital Photograph]. Retrieved from http://edition.cnn.com/2016/06/20/arts/the-hive-wolfgangbuttress/

Figure 6.2. Buttress, W. (2017). Design Elements (Art Installation) [Digital Photograph]. Retrieved from http://edition.cnn.com/2016/06/20/arts/the-hive-wolfgang-buttress/

Figure 6.3. Buttress, W. (2017). The Hive Interior with Visitors (Art Installation) [Digital Photograph]. Retrieved from http://edition.cnn.com/2016/06/20/arts/the-hive-wolfgang-buttress/

\section{Page 30}

Figure 7.1. Noble, A. (2015). Dead Bee Portrait \#01. In No Vertical Song. Auckland, New Zealand: Two Rooms Art Gallery, 28 May-4 July 2015.

Figure 7.2. Noble, A. (2015). Eidolon \#5 [Pigment print on Hahnemühle paper, 1200 x 800 mm]. In Nature Study. Wellington, New Zealand: Bartley \& Company Art Gallery, 4-28 February 2015.

Figure 7.3. Noble, A. (2015). Bee Wing Morphology \#6 [original tintype /silver emulsion on coated aluminium, $300 \times 245 \times 65$ mm]. In Nature Study. Wellington, New Zealand: Bartley \& Company Art Gallery, 4-28 February 2015.

Page 69

Figure $19 \& 20$

(Wellington Beekeepers Association, Personal Communication, May 29, 2017)

\section{Personal Photography \& Design Graphics}

Figure 1 (Page 9)

Figures 2 \& 3 (Page 14)

Figures 8-18 (Page 53-68)

Figures 21-24 (Page 71 \& 72) 


\section{Introduction}

Creating A Buzz aims to address how communication design can be used to raise awareness regarding relationships between Wellington's honeybee ecologies and the sustainability of our local environments. In this thesis, concerns of human interactions, beekeeping practices and the well-being of honey bees are considered through Research About Design. The value of this topic is indicated by the current size and economic value of New Zealand's honey and related trade industries, along with global concerns of bee decline from external influences such as climate and agricultural farming practices. There are currently growing interests in beekeeping and bee concepts as urban beekeeping practices are become more popular in central and suburban Wellington. The engagement of public audiences aims to encourage positive environmental actions in local communities and looks to foster an understanding of the importance of our local ecosystems and bee species. This includes encouraging the spread of knowledge and educational activities that can benefit bees, without having to physically interact with them. This thesis aims to consider a holistic understanding of current issues and concerns raised by key stakeholders, and how these can be addressed, solidified or altered through design in future Wellington contexts. Key issues to address are; the resilience and sustainability of our environments, raising awareness about encouraging growth in our ecosystems, and showing how communication design can be used to portray these ideas.

\section{Literature \& Design Review}

Supporting the thesis aims, the literature and design review includes current examples of published research, and visual and installation designs that focus on the impact of honey bees on local environments. The literature focuses on the key ideas realised by the state of Wellington's bee population health and explores approaches to various local environment and bee-related concerns. The context of Wellington is defined, and an urban setting is included to support the current rise in urban beekeeping. Wellington's current resilient and sustainable plans, including 'Global Sustainable Development' and '100 Resilient Cities' have been implemented to instruct pathways for future environmental concepts and actions. The key concepts from these plans have indicated a national eco-city focus, promoted through initiatives towards: economic growth, social inclusion and environmental protection. With communication design techniques addressing design for the education of the public, avenues of design are then evaluated to determine the correct application of ideas and output type. The design precedents demonstrate how communication design methods can be applied to honey bee related concepts, and the purpose of raising awareness through public installations.

\section{Research Methodology}

From the identification of key research and design reviews, the research methodology structures the appropriate techniques and processes to follow throughout the duration of the thesis. Research About Design methods includes the importance of design research, a human-centered design approach and visual research ideas. This then informs the qualitative data collection and the recruitment process, including representatives from: Victoria University Biology Department, Wellington Botanic Gardens, Wellington Beekeepers Association, and the Beeple Honey Collective. Semi-structured interviews with the recruitments aims to collect perspectives and opinions on current barriers and concerns about Wellington's bee populations and how current information and education is portrayed to local audiences. An analysis of these interviews evaluates stakeholder perspectives and formulates design suggestions, as well as offering a structure of key issues to include. The application of these methodologies informs the design development phase and incorporates communication design principles in the pursuit of a final outcome. Research Through Design includes the iterative design process, concept testing with stakeholder feedback, and the development and refinement of key proposed visuals and content. These instructions lead and develop the final form and target audience for the design, with the website prototype foreseeing future extension through related media platforms and physical location interactions. 


\section{Stakeholder Perspectives \& Design Recommendations}

Realising the need for expert opinions and current industry information leads to the undertaking of four separate semi-structured interviews with stakeholders from Wellington organisations. This process involves a range of representatives with academic, beekeeping, conservation and community knowledge and experience. Commonalities of current industry concerns were addressed through these interviews and documented, showing particular concerns surrounding the cultural perception of the bee, the correct management of hives, current education platforms and content, and environmental barriers. Forms of online content and sharing of information is one of the main educational focuses, with discussions about organisation-resident interactions including: sharing through websites, Facebook, and community projects/events. From the interviews, primary commonalities are identified and applied to the formulation of a communication design criteria which illustrates how to visualise these ideas, and how to create a cohesive message that can be targeted towards the correct audience. Through suggestions from experts, current target markets in Wellington include younger generations, particularly primary school children and education programmes. This is why the chosen target market for this design focuses on students and young professionals in Wellington, who are understood to be the next generation of bee caregivers in Wellington City. The decided outputs of social media platforms and a website prototype are ideally suited to this target market as they are commonly the largest users and consumers of these media types. This consideration leads into the design process, as the research and design methods, key ideas, target market and outputs have been previously researched and justified.

\section{Design Process}

Through the design process, all data collected and research conducted is synthesized into a comprehensive website prototype which prioritizes visual media, and photography in particular, as a tool for raising awareness and communication. All content and research previously conducted is utilised, with the addition of visual components - specifically the media of photography. The photographic elements aim to explore a different perspective of the bee, one that gives an insight into a larger scaled world and the scale at which they would interact with their surrounding environments. A communication design and website criteria is formulated from communication principles which aim to address the purpose of design for the education of the public. The design iterations focus on the aesthetic of a functioning website, with online interactive notions designed to imagine a real life scenario. Critical feedback from key stakeholders enables the information and images to be refined and explicitly state whether the content is worthy and suitable for presentation. Consideration of the potential interaction at physical locations is designed through the integration of QR codes at public beehive locations, e.g. the two beehive locations at the Wellington Botanic Gardens. This enable a direct link to the online prototype with more opportunities for information viewing and sharing among social media platforms. The social media platforms (Facebook and Instagram) allow for specific locations to be tagged, which are then collated on the website to demonstrate the physical community involvement through images online. Collectively, these platforms aim to illustrate the meaning of human interactions with our local bee populations and how we can visually represent and take actions towards encouraging their well-being, growth, and future existence in Wellington. 


\title{
Motivation \\ Trevor Charles Ottaway 30/07/1923 - 02/04/2002 Grandad \& Beekeeper
}

\begin{abstract}
When my mother was growing up in and passionate about the bees' place in Gisborne (mid 1960s) Granddad Trev the world, especially our local ecosystems. and his father Alfred Zion Ottaway ran a As a child, I remember observing Grandad beekeeping business. My mother used to inspecting the hives at the farm and tasting work alongside them in the honey shed, the freshly extracted honey. We now helping with the extraction of honey from the have beehives at our own home in central frames and then decanting the honey into Auckland as a consequence of growing up jars for sale. With Alfred passing away during around them and being knowledgeable that the late 1960s and a change in the health the species is under threat and the vital role regulations for food handling, Grandad they plan in mankind's existence. Observing Trev stopped selling honey commercially. and learning about Grandad's true devotion He then continued as a beekeeper with a to honey bees, has inspired me to continue small number of hives on a friend's farm and this legacy through my own talents and he was paid to place his hives in kiwifruit contemporary ideas. With our current orchards and among squash crops for climates changing so rapidly, it is important pollination. The honey extracted from these that these historic beekeeping practises are hives was distributed to close friends and not forgotten and are used to help prevent family. He was extremely knowledgeable future honey bee extinctions.
\end{abstract}




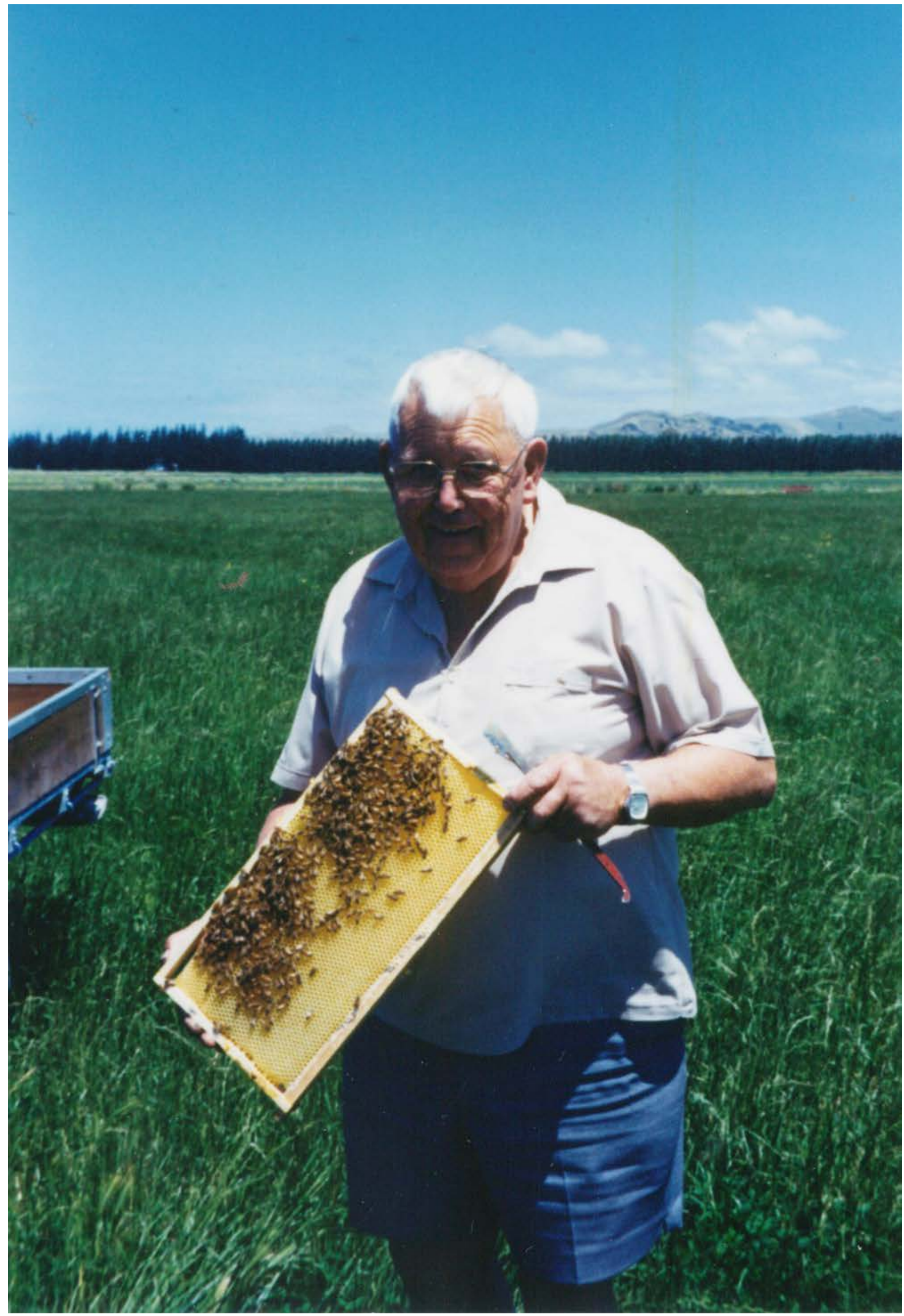

Figure 1. Grandad Trev and his bees in Gisborne, by Author 


\subsection{Literature Review}




\section{Introduction}

The honey bee acts as a symbol that connects humans, more-than-human populations and Wellington's environmental sustainability and resilience. Both humans and honey bees rely on the sustainability of Wellington's natural environment, co-existing in communities with large populations, and must strive to achieve survival in an increasingly urbanised ecosystem. The importance of other living species in our environments needs to be recognised among local sustainability plans, which should aim to encourage the well-being and contributions of honey bee populations. The intensity of honey bee population decline has been realised on a global scale, through ongoing losses of natural crop productions and produce industries. In order to prepare for future living scenarios, we must first identify and address the needs of our local populations. As bee populations are one of the only existing natural pollinators, their role in sustaining the growth of surrounding subecologies is crucial to Wellington's ecological and biodiverse system. Currently, declining honey bee populations worldwide is primarily the result of the growth and well-being of their ecosystems being threatened. The honey bee is one of the biggest contributors to our natural resources as well as the provider of a commercial product industries that influences New Zealand's economic state. They support food productions, the pollination of native flora, and ultimately play an essential role in maintaining a thriving ecosystem. The resultant declining domino effect has the potential to lead to substantial losses in food and consumer industries. For instance, detrimental to New Zealand alone, NZ Federated Farmers (2016) has identified that "pollination provided by the bee industry contributes at least $\$ 4.5$ billion annually to New Zealand's economy and underpins a further $\$ 12.5$ billion of export revenue for the horticulture, arable, pastoral and beekeeping sectors". With the honey bee providing such environmental benefits and contribution to a diverse range of industries, their relevance to New Zealand requires us to focus on providing adequate care that solidifies their growth and existence in future contexts.

Wellington City's current 'Global Sustainable Development' and '100 Resilient Cities' implementation plans have begun to establish potential opportunities for future human contribution to the conservation of our global environments. Solutions for global climate concerns and trends have been established and reinforced by the United Nations, 2030 Agenda for Sustainable Development. This policy intends to involve global communities that promote and practise initiatives towards three core elements: economic growth, social inclusion and environmental protection. Local strategic goals have also been identified by the Wellington City Council (2016), through the '100 Resilient Cities' platform, which seeks to achieve a complete eco-city integration into urban areas. As more initiatives are developed to ensure the well-being of sustainable urban environments, the required human actions of Wellingtonians are being critiqued and improved upon.

In an effort to address New Zealand's bee population specifically, in March 2017, Auckland City Council introduced a funded art project called 'For the Love of Bees' that aims to turn Auckland into the world's safest city for bees. The major priority of the project stands with the health, wellbeing and happiness of the hives in central Auckland, with honey production being a secondary concern. The campaign introduced a bee colony of 5,000 bees to Auckland's Victoria Park, which will stay for the next two years, eventually growing into a colony of about 150,000 (Pasley, 2017). To provide a sustainable food source for the bees, parks and reserves are being connected by bee-friendly planting, creating targeted 'pollination pathways' with these connections being supported by free weekly sessions about seeds and sustainability. The campaign is aimed at creating a "safe inner city ecosystem for bees by educating people about what seeds they should plant, introducing more hives and planting more beefriendly flowers throughout Auckland" (Pasley, 2017). For the Love of Bees organisation (2017) explains that "each of these hives needs 140 million flowers to visit to make the honey they will need to have a healthy colony. This is why we have established a biological/organic teaching garden at Griffith Gardens so that everyone can come and learn how to grow the 840 million flowers the Bees in Parks will need." Along with the Victoria Park habitat, the project artist Sarah Smuts-Kennedy says "we will also have hives in Myers Park as well which means we will have 300,000 more bees in City Parks by the end of the year." Targeted at the benefit to Auckland's inner city population, the greatest lesson learnt from this project is that bees can thrive in urban environments. The implementation of this project sheds light on the current education tools needed for local communities and individuals to help bee populations thrive in their natural environment. 
This Auckland bee project was invented and constructed by a Fine Arts student, and is an example of how current concerns and issues regarding honey bee populations can be addressed through communication outputs. The central location of the hives addresses public audiences and physical interactions in Auckland's urban areas, with community parks being used as the showgrounds for these bee habitats and planting initiatives. The practice of communication design enables these issues to be addressed through principles that directly target design for the education of the public. Communication mediums are the ideal tool to design information that is suitable across multiple platforms and can act as an educational resource for the public. The relevant ideas about the importance of honey bees in New Zealand and their influence on our surrounding environments and future sustainability can be targeted through communication design outputs with the intent of raising public awareness.

\section{Honey Bee Populations}

The initial introduction of bee species to New Zealand began in the 1830s, with eight different species deliberately introduced, including the honey bee (Apis mellifera). The purpose of their introduction was to pollinate either red clover (Trifolium pratense) or lucerne (Medicago sativa). The honey bee was first introduced from England into the North Island of NZ in 1839, then followed by their introduction in 1842 into the South Island from Australia. Howlett \& Donovan (2012) indicate that "of the 40 species of bee within New Zealand's biogeographical region, 13 have arrived since European settlement with 8 having been deliberately introduced to aid crop pollination". The resultant growth and development of the bee industry in New Zealand is substantial, as NZ Federated Farmers have identified "pollination provided by the bee industry contributes at least $\$ 4.5$ billion annually to New Zealand's economy and underpins a further $\$ 12.5$ billion of export revenue for the horticulture, arable, pastoral and beekeeping sectors" (Federated Farmers, 2016). The importance and contribution of this "sector holds such a responsibility; pollination delivers much of the food we eat and a large portion of the products which we export, as a nation" (Federated Farmers, 2016). There is no other sector in New Zealand that creates such a diverse market of opportunities for creation and consumption. The importance of bees pollination, combined with the production of honey by-products, underpins the scale of their contributions for human benefit. The importance of our understanding the effects of human activity on honey bee ecologies can indicate the well-being of surrounding ecosystems, and how these production systems relate.

Historically, the introduction of honey bees to New Zealand was valued by both indigenous cultures and resources. Due to the vast expanses of land turned over to farming "following the First and Second World Wars, the number of beekeepers and beehives increased considerably as land was cleared and developed for agriculture, shaping what would eventually become a flourishing national industry" (Beard, 2015, p.4). Until then, the first introduced honey bees had remained feral and unmanaged, and "had become plentiful in New Zealand's indigenous bush areas as early as the 1860s and considerable quantities of honey were being sold by Maori" (Gillingham, 2012). The influx of bee populations spread rapidly among the North and South Islands as feral honey bees, at their peak "were estimated to number between 10,000 and 50,000 on each island contributing to a large proportion of the pollination services for pastures and horticulture crops" (Goodwin, 2010). Beard (2015) explains the benefits to native resources also "underpins New Zealand's excellent international reputation and export market for a range of highquality monofloral honeys (i.e. honeys that are predominantly sourced from a single indigenous plant species)" (p.5). This pristine reputation means that "indigenous forests are highly valued by beekeepers for the clean nectar and pollen sources that they provide (i.e. free from the chemical toxins that are often associated with food crops), which is an important requirement for maintaining and rebuilding the strength and health of managed bee colonies" (Beard, 2015 , p.5). In order to protect the pureness of our environments and honey bee populations "various imports of live bees occurred up until the 1980s, when the practice was halted to protect New Zealand's bee health status" (MPI, 2014). As concerns grow regarding bee health and well being, future controls are expected as "all domesticated beehives in New Zealand now rely on human interventions for survival" (Goodwin, 2006). This shows that while, previously, we relied on the environmental contributions of bees to support our industries and global reputation, now they, in turn, rely on human intervention for guidance and survival. The mutual benefits from both historic and current relationships in New Zealand, further justify the concerns associated with honey bee survival and their environmental conditions and treatment. 
Figure 2. Family beehive in our backyard at home in Auckland \#1, by Author.
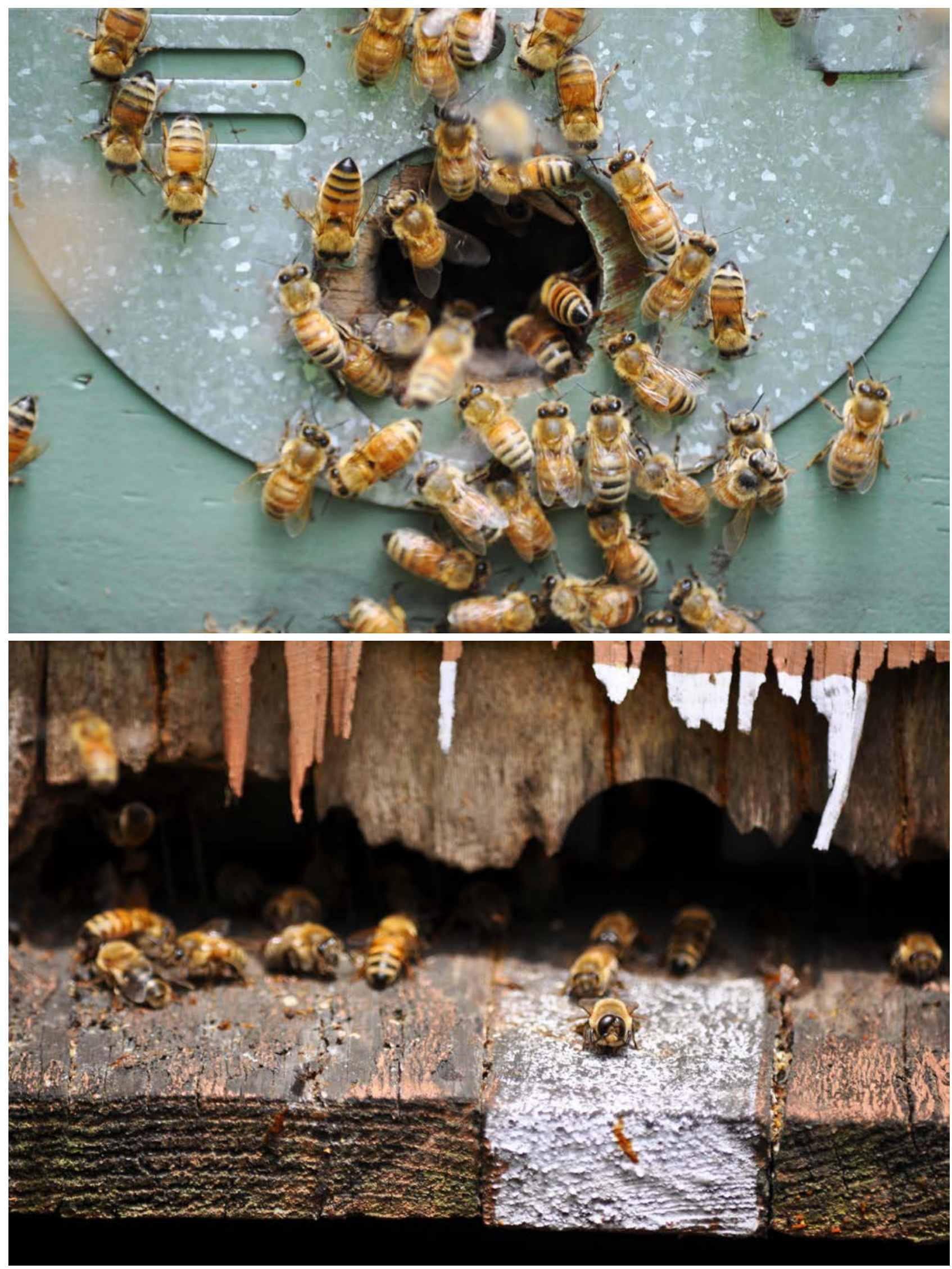

Figure 3. Family beehive in our backyard at home in Auckland \#2, by Author. 
The overall reality of honey bee population decline is due to many contributing impacts such as climate change, viral diseases, harmful pesticides and mistreatment through mass agricultural farming. The declining nature of honey bee populations becomes rapid as responses by beekeepers are relied upon to eradicate any harmful influences. The current knowledge and expertise of beekeepers can either interrupt or encourage honey bee growth in hive-based locations, with specific observations being key in interpreting unique issues. The major viral disease, varroa mite, established in New Zealand in 2000 is "predicted to have an economic impact of \$365-\$661 million between the period 2003 to 2028 and between $\$ 198$ and $\$ 433$ million dollars in the South Island alone" (Howlett \& Donovan, 2012). NZ Federated Farmers indicates, "aside from habitat loss, the main issues leading to a pollinator crisis in New Zealand are increasing honey bee pests and diseases, declining floral resources leading to pollen shortages and bee malnutrition and undervaluing the vital role of the beekeeping industry to the economy" (Federated Farmers, 2016). The particular focus on declining floral resources and bee malnutrition will be key influencers throughout the communication design process. As solutions already exist regarding the spread of varroa mite, new ways need to be identified to create solutions for other main issues, all contributing to the overall environmental sustainability and the well-being of honey bees. Creating a shared responsibility among residents, for example, identifies that "it is vital as a country we recognise the immense value the honey bee delivers and accordingly take immediate steps to strengthen our protection of this key sector" (Federated Farmers, 2016).
The prominence of Wellington's beekeeping practises advances the recognition and local expertise regarding honey bee populations and general hive wellbeing. Continual maintenance and concerns are demonstrated predominantly through the Wellington Beekeepers Association's online platforms and community meetings. In order to relate to the current urban environmental trends, NZ Federated Farmers wants a "recognition of the value of bees to New Zealand as a whole to the country and the economy, and ongoing financial support to be made available to the bee industry for the continuations of surveillance, education and bee research" (Federated Farmers, 2016). Urbanisation observations throughout recent decades, indicated by Goddard (2010), show that "research in urban environments has embraced the ecology-of-cities paradigm: an interdisciplinary approach that views urban landscapes as socio-ecological systems within which humans and their social institutions are integrated with the environment" (p.92). This concept relates to the habitat interconnectivity of our ecological systems, which poses concerns related to current honey bee maintenance and habitat loss. The survival of honey bee ecologies currently relies largely on the guidance and control of beekeepers caring for beehives in central Wellington, alongside other uncontrollable factors such as weather and habitat conditions. A response to the importance and education of beekeepers must be reliable, as they are imperative to the survival of honey bee populations. Regarding the well-being of bee species, assessed risks around introduced populations must "consider the requirements for a continuous and high-quality food supply for indigenous species in order to support population survival and fitness" (Beard, 2015, p.8). To sustain Wellington's current urban environment, "careful consideration needs to be given to the timing of beehive placement relative to flowering peaks and critical life-stages of indigenous fauna, and to the number of beehives that may be appropriate for any given area" (Beard, 2015, p.8). 


\section{Global Sustainability}

Ideally, honey bees need warmer temperatures, lots of sunlight and less wind and rain to enable them to function on a daily basis. Uncontrollable elements such as weather and habitat conditions affect on the consistency of the climate and the bees ability to cope with climate changes. This means that any extreme weather conditions can halt the bees natural processes and, with global climate conditions becoming more prevalent, specific bee limitations will result from the damage climate change is having on bee populations. Current initiatives addressing climate change issues were introduced in September, 2015 by the United Nations, in the 2030 Agenda for Sustainable Development. Sustainable development, defined as "development that meets the needs of the present without compromising the ability of future generations to meet their own needs" (United Nations, 2015). Specific goals aim to reach "all countries and all stakeholders, acting in a collaborative partnership," as the realisation for a greater scale management followed on from the successes of the Millennium Development Goals (MDGs). These targets have become "global in nature and universally applicable, taking into account different national realities, capacities and levels of development and respecting national policies and priorities" (Sachs, 2012). The shared responsibility of performing actions in order to improve the planet for future generations, has evolved from the immense scale of concern throughout all social, economic, and environmental dimensions of sustainable development goals (SDGs). These 17 SDGs are accompanied by 169 specific qualitative and quantitative objectives, with numerical indicators being developed to test the progress and effectiveness of each goal.
The encouraging 'pro-environmental behaviours' definition by Steg and Vlek (2009) "refers to behaviours that harm the environment as little as possible, or even benefit the environment" (p.309). Current threats to environmental sustainability, including "urban air pollution, environmental noise and loss of biodiversity, many of these problems are rooted in human behaviour" (Steg \& Vlek, 2009, p.309). Barr (2003) suggests that a "solution to this problem is often seen to lie in the provision of information and knowledge dissemination" (p.227), regarding the prediction of sustainability. An ongoing evaluation and problem solving indicator shows "the challenge is to understand the cognitive, motivational and structural factors and the processes that threaten environmental sustainability, so that pro-environmental behaviours could be facilitated and emerge worldwide" (Barr, 2003, p.315). Informative design strategies illustrate changes to moral and normative concerns, habitual behaviours, and environmental motivations. One major response to consider is assuming the "belief that individuals and institutions can be persuaded to accept responsibility for the production of environmental problems and change their everyday practices to alleviate future impacts" (Burgess, 1998, p.228). Grainger, Mao and Buytaert (2016) propose; "by providing alternative future conditions, choices and possibilities, scenario planning and analysis are useful tools to deal with uncertainty, especially in an era of uncertain trajectories of global change" (p.303). 


\section{Wellington Eco-city Focus}

Wellington's 'Towards 2040: Smart Capital' plan illustrates actions and goals towards becoming a fully functioning eco-city, by involving suitable approaches to environmental change and improvement. Richard Register's (1987) book, Ecocity Berkeley: Building cities for a healthy future originally documented the term "eco-city" as a "living system with a land use pattern that supports the healthy anatomy of the whole city, enhances biodiversity, and makes the city's functions resonate with the patterns of evolution and sustainability" (Register cited in Wong, 2011, p.3). One of the main goals documented by the Council is to protect Wellington's green infrastructure, including the Town Belt, to support biodiversity and offset carbon emissions. The additional goal, to involve everyone in a citylevel approach to Wellington's future as an eco-city, combines the two systems of human involvement with ecological contributions. Wellington's inclusion in the 100 Resilient Cities initiative aims to realise environmental practices that can be applied to future scenarios, involving and educating the actions of current residents. This relates well to education about the integration of honey bee populations, specifically concerning the role of honey bee populations and how these populations are currently being managed. "Resilient Wellington is part of a wider global initiative bringing together 100 cities working on improving their resilience through shared analytical tools, processes and most of all through shared ideas and lessons learnt" (Rockefeller Foundation, 2017, as documented by the 100 Resilient Cities, 2017). One of the main observations in Wellington city is the need "to adapt our people, infrastructure and institutions to our changing built, natural and economic environments, particularly the need to adapt our city to the effects of a changing climate." The integration and access of people in Wellington, becomes a crucial aspect in achieving momentum towards urban sustainability.
According to world land mass statistics, Wellington city includes a comparatively high percentage of open spaces, documented at around $48 \%$ of overall land. The strategic city plan supports important biodiversity, carbon-reduction, landscape and recreational benefits, and increases growth potential for our ecological networks (Towards 2040: Smart Capital). The direct availability of natural resources is imperative to honey bee colonies as it facilitates the transportation of pollen and the fertilisation of crops across connected landscapes. The interdependency of human and non-human species and the 'rights' and 'intrinsic values' must also be included when considering environmental issues, as landuse planning can no longer afford to be purely human-centered. Wong (2011) stresses that global concerns regarding "rapidly rising urban populations, relatively low levels of environmentled technologies, management and awareness in environmental protection all contribute to the urgency for action" (p.1). The construction of Wellington's urban landscape enables an integration of ecological networks, that are reliant on the close proximity of native and exotic plant vegetations. To consider a pursuit towards a sustainable ecosystem, the focus has to become ecological, with concerns focused towards urban planning and future sustainable environments. Critical interactions between Wellington residents and their surrounding flora and fauna needs to take into account the future growth and placement of urban populations on more land areas. The development of urban spaces for greater population growth must integrate further communal areas for inner city species. All considerations must include effects on the life-cycles of our landscapes, ecological systems, and human populations in order to understand the interrelation between their existences and reliances on one another. 
Lundquist (2011) indicates that continual climate changes heavily impact our ecology as "New Zealand is one of the global hotspots for biodiversity due to its high endemism, and potential threats to fauna and flora" (p.180). Biodiversity is further restricted, as "about $78 \%$ of terrestrial habitats have been extensively modified through conversion to agricultural, pastoral or urban landscapes" (p.180). Lundquist identifies that, "the most serious threats to New Zealand's biodiversity and ecosystems involve interactions between climate change and pre-existing threats such as habitat loss and fragmentation, and invasive species" (p.186). Goddard (2010) documented current trends in ecology and evolution and determined that "as urbanization increases globally and the natural environment becomes increasingly fragmented, the importance of urban green spaces for biodiversity conservation grows" (p.90). In order to understand connections of growth and sustainability "we will need to develop more urgent responses to protect our biodiversity, and gain a better understanding of the relationship between our urban and natural environments" (Towards 2040: Smart Capital). Resilience to environmental changes needs to become a critical and flexible approach, considering future trends can only be predicted, not confirmed. Relating the impacts of biodiversity to the input of humans requires an empathic view of the environment and the ability to notice and implement feasible actions. The focus on urban environments becomes highlighted when realising the occupancy of industrialised humans on native land, who often have a better understanding of other species and the need for coexistence. Lundquist (2011) proposes; future advanced plans and infrastructure must include "other adaptation plans to ensure our communities and ecosystems are as resilient as possible in the face of uncertain changes to New Zealand's climate" (p.188).

The importance of relating the impacts of climate change and resilience strategies on honey bee populations, raises concerns for the overall pollination and survival of sub-ecologies and crops. A mutual existence of ecosystems involves determining how relationships can interact and alter throughout the process of evolution. Considering local environments, the 'Smart
Capital' plan identifies that "we need to build our resilience to the impacts of climate change and protect our natural resources" (Wellington City Council, 2016). In conjunction with a resilience plan; "we need to understand and develop the economic potential of our ecological assets through research and innovation" (Wellington City Council, 2016), to recognise the ability design has to expose the capabilities and constraints of our urban environments. A central focus into the beneficial ecological contributions of the honey bee, accentuates the importance of healthy interactions between inner city human and non-human populations.

In light of the increasing urbanisation and population growth among developing cities like Wellington, the sustainability of honey bee ecologies is a priority. Grimm (2008) proposes that urban concerns should change an environmental focus on "material demands of production and human consumption that alters land use, biodiversity, and hydrosystems locally to regionally" (p.756). A careful consideration of empathic human and non-human relationships is imperative to understand future influences on surrounding biological systems. A sustainable focus stabilises future environments, whilst fully encompassing the growth and biodiversity of native species. Brown (2016) considers that, "how we understand and recognise our relationship with nature is reflected in our approach to sustainability. As the degree of this relationship weakens, so does our respect for nature and consequently our tolerance for doing harm" (p.31). Effective promotion of empathic relationships addresses the need for greater environmental awareness across Wellington City. This includes the existence of individual human impacts alongside the occupancies of developing structures and buildings, engrained in Wellington's overall structure and orientation. Grimm (2008) insists "urban ecology at the local scale centers on the relationships among urban design and constructions, ecosystem services delivered in the new system, and responses of people to evolving opportunities" (p.756). The importance of seamless correlations between honey bee species, shared ecosystems and urban configurations allows for a design implementation that recognises the importance of New Zealand's bee populations. 


\section{Communication Design}

In order to relate directly to the well-being of honey bee ecologies, communication design needs to provide an opportunity to raise awareness and disseminate information and sustainable tools in physical and online environments. Through the power of highly accessible and interactive visual media harnessed in communication design, empathy and engagement may be cultivated. Communication design offers an ideal means to promote these issues. Further, the exposure of current sustainable beekeeping practises and knowledge within Wellington can be portrayed through design which reinforces the sustainable follow-on effects of honey bee activity in our environments and communities. Incorporating an understanding of the current relevance of honey bee populations in New Zealand will aid in structuring the type of designed information conveyed to the public. This research aims to connect the important relationships concerning Wellington's honey bee ecologies, so that adequate and ethical care can be maintained. The collective output of communication designs aim to raise public awareness towards our shared ecological condition as it relates to climate change and the critical need for bees in our native ecosystems.

\section{Communication and Communication Design}

Communication is an invaluable, yet complex tool and its application must be approached with great consideration, specifically in relation to the use of common language and the boundaries between human and more than human species. Grainger, Mao and Buytaert (2016) caution that "within the interface between science and society, indifference towards communication prevents comprehension, creates misunderstandings and inconsistent or bias messages" (p.300). An overall conclusion from their investigations is that "there remains a gap between scientific knowledge generation and non-scientific, societal application" (2016, p.300). The overcoming of boundaries between species is well documented by Donna Haraway, who explains that "transspecies encounter value is about relationships among a motley array of living beings" (Haraway cited by Moore \& Kosut, 2013, p.210). Haraway (2008) proposes a behavioural change among people "propelled by the tasty but risky obligation of curiosity among companion species, once we know, we cannot not unknow. If we know well, searching with fingery eyes, we care. That is how responsibility grows" (p.287). Moore and Kosut (2013) give urban beekeepers a social purpose by describing them collectively as "a very interesting tribe who deliberately and intentionally pursue interspecies relationships with insects - their relationships emerge from soulsearching, altruism, commerce, activism, and community building" (p.209). There is potential to learn from current beekeepers' relations with honey bee populations, to understand the vulnerabilities and behaviours of the species. In order to correctly portray the honey bee's relevance to our environments, public education and future honey bee information will be influenced by current beekeeper interactions and understandings. Moore and Kosut (2013) have a particular desire "to understand our fellow urbanites in their yearning to "connect with nature'" (p.209), proposing a direction for more-than-human integration, furthering the opportunity to purposefully raise awareness and fostering empathic understandings.

The intended aim of integrating Wellington audiences provides opportunities for knowledge sharing and personal environmental awareness, alongside specific honey bee interactions and behaviours. The combining of information and new communication tools has the ability to influence positive sustainable attitudes and behaviours. Overall evaluations from a wide range of industry experts will allow for multiple perceptions to inform the current challenges and concerns of the honey bee industry, which can then be addressed through design. Visualisations and various media platforms aim to entice individual viewers to promote and practise sustainable solutions for the benefit of honey bee health and well-being. The technological aspects become important when considering the source and portrayal of information, and whilst considering the interactions these applications offer. To implement an effective outcome, the information and design must be concise and appealing to contemporary local audiences, by contextualising the location and role of the bee in Wellington in terms which resonate with the intended audience. Grainger (2016) rightly observes that "from a user or audience perspective, visualisations should increase understanding; and ultimately, enhance their ability to complete a task or make an informed decision" (p.301). Applied well, communication design has the ability to convey perceptions of Wellington bee organisations that structures persuasive and meaningful educational tools for targeted audiences. 
Through the eyes of the communication design creator, Jorge Frascara (2004), "to design is to invent, to project, to program, to coordinate a long list of human and technical factors, to translate the invisible into the visible, and to communicate" (p.2). Jorge Frascara, in 1997, introduced the term 'communication design' as one which associates with multiple disciplines and graphic styles, originally formed from the graphic design profession. Visual communication, closely related to graphic design, falls under the broader scope of communication design as it relies on the visual output of products and ideas. The words 'visual communication' modify the word 'design', and relate it to the production of visual objects aimed at communicating specific messages. The visual aspects have been narrowed in, due to its close relation to intended outputs. Visual communication, "seen as an activity, is the process of conceiving, programming, projecting, and realizing visual communications that are usually produced through industrial means and are aimed at broadcasting specific messages to specific sectors of the public" (Frascara, 2004 , p.2). In order to confine the definition to a professional design field, Frascara (2004) argues that "the purpose of communication design is to affect the knowledge, attitudes, and behavior of people - something that happens after the communication takes place" (p.13). The particular goal of impacting public knowledge makes it clear that the visual communication of ideas can be imperative when creating an understanding or pitching a potential change in behaviour to a larger audience. The best application of ideas can be portrayed and effectively contribute to implementing the intended output, with specific consideration to the context and receiving audience.

In order to specify the requirements and criteria applied to a design output, an integration of different communication practises needs to be identified and considered. In Jorge Frascara's 2004 publication, the most appropriate term documented for the intended design outcome is 'design for the education of the public', as "it works in methodological terms through the three types of design already discussed: information, persuasion, and education" (p.157). This is due to multiple components of design collaborating in an effort to design for a particular focus towards raising awareness. The three components of information, persuasion and education are broad indications of design, when paired with public persuasion, directs the specific purpose for overall design considerations. The specific output focus of data visualisation is achieved by integrating a significance in graphic and technological forms across various multimedia platforms. The end communication design "expressed in this definition states that data visualisation is about facilitating understanding" (Kirk, 2016, p.21). In order "to derive understanding from data we need to see it represented in a different visual form" (Kirk, 2016, p21). The act of data representation allows a spectrum of personal opinions to formulate, based on context specific facts and examples. Inclusive of the data visualisation role, the concept of "pragmatic design" (Katz, 2012), enables the user to understand and interpret data. Kirk, alongside Katz (2016) identifies "an important reason for giving greater weight to pragmatism is because of people: people are the makers, the stakeholders, the audiences and the critics in data visualisation" (p.7).

In line with Wellington's Towards 2040 plan, intended audiences and responses are invited to consider the aims reflected by the council. One main aim identified is to 'support participation and engagement through increased transparency and accountability of city-level decision making.' Specifically noted is the need to include active communities that support innovation and resilience. The technological design output relates to the wants regarding the use of 'ICT and social media to augment traditional forms of engagement to increase access and relevance of city and communitylevel activities'. The engagement of communities through online platforms of information allows an unlimited reachability of data, outside of the main context of Wellington. The application of the information to Wellington allows a potential direction for communication design to become a resourceful avenue to process and display information for public audiences. The ability to revise content and generate creative content on engaging platforms, encourages interaction and understanding of complex issues. The intended Wellington audience is encouraged to revisit the importance of inner city working populations and family-based residents, in combination with increasing further education and awareness throughout local communities. A specific design direction to integrate public strategies and information will be focused through the intended outcome of changing behaviours and attitudes. 


\section{Conclusion}

The development of a platform that can effectively address the issues faced in Wellington's contemporary bee populations requires an ethically centered strategy. As proposed by Moore \& Kosut, such a strategy involves creating more deliberate and thoughtful working conditions for bees that consider their health and well-being. A potential direction begins to extend understandings into how humans talk about and regulate bees. This can also be used, expanded, and perhaps reconceived through considering relationships between humans and nonhumans (Moore \& Kosut, 2013, p.173). In relation to urbanisation and sustainability, Moore suggests that we need to become more aware of our ecological inertia and complacency and be more critical of buying into symbolic green consumerism. The manner in which we engage with bees is being questioned through ethical engagement which encourages modifications in our practises and relationships with bees; it seems to us that this speaks to contemporary notions of sustainability. Current information regarding honey bee populations needs to be directed in a manner in which they are not taken for granted as an inexhaustible natural resource. Moore and Kosut (2013) believe that we must position bees in relation to us, not as mere insects but as a highly valuable species that must be protected. Properly directed, beekeeping practices are able to inform types of positive interactions with bees, whilst carefully considering their location and their environmental purpose. Moore and Kosut reinforce this perception of beekeepers in saying, "collectively we pin our hopes on beekeepers as a sign that human and nonhuman lives will prevail in environments choked by human progress, consumption and desire. Perhaps humans have not simply domesticated bees; instead, they have created a new place and social space for the city farmer" (p.214).

Globally, there is a greater personal disconnect and lack of responsibility towards actions that create a positive environmental change and in recognising the need for a greater importance in sustainable and ethical human interventions. Brown (2016) suggests that "we can and must reignite sustainability, set the sustainability soul on fire, make sustainability fun and exciting, and inspire a new generation, acknowledging the damage of the past and make amends, healing the future" (p.27). A future direction breaks down opposing relationships between humans and nature, and combines a radical and ethical engagement in prevailing environments. The recognition and acceptance of this relationship is reflected in our understanding and therefore our approach to sustainability. As the degree of this relationship weakens, so does our respect for nature and consequently our tolerance for doing harm (Brown, 2016, p.31). The objective of raising awareness in order to foster empathic relationships, requires influencing the behaviours and actions of local urban Wellington populations. The willingness for communities to unite and to see change as necessary will be reflected in the development and understanding of ethical bee interactions and the personal relationships these foster. The health of the honey bee becomes reflective of the individuals in a community, their survival is dependent on the willingness and nurturing of beekeepers. The passion and interest of these individuals will drive the growth of bee populations and set up a network of professional mentors with trusted skills and experience to lead the future practices in beekeeping and the engagement of the wider community in the maintenance and care of the bees' environment.

Understanding our reliance on nature needs to direct the opportunity for communication design in a core way, to sustain local and global conversations. The various manifestations of communication design target education through visual stimulus extended on a public platform, to engage a variety of audiences. This design output aims to provide a new vision and narrative for sustainability. As Brown (2016) suggests, "we can and must rethink and develop a new, shared understanding of sustainability, with new stories, a new language and new thinking" (p.31). The empathic perspective provides a different approach in the outputs intentions, with an understanding of the importance of honey bee populations helping to broaden the tolerance for critical issues and acting as a symbol for positive change. The overall design intentions aim to foster an understanding and create a future critical direction for these particular sustainability issues, by enabling an insight into our collective and individual human impacts on nature. Engaging local communities targets the importance of individuals connecting and having a voice in the future of our societies. Supporting actions of change, whether small or large, creates a collective responsibility to act upon the threat of extensive honey bee decline. The capabilities of being able to aid in this problem, by providing individual steps to a greater solution, reinforces the dedication humans need to have in order to support the wellbeing and growth of New Zealand's bee populations. 


\subsection{Design Precedents Installations \& Photography}


Figure 4.1. Park Pop Project, by Smuts-Kennedy, S., Tairoa, T.

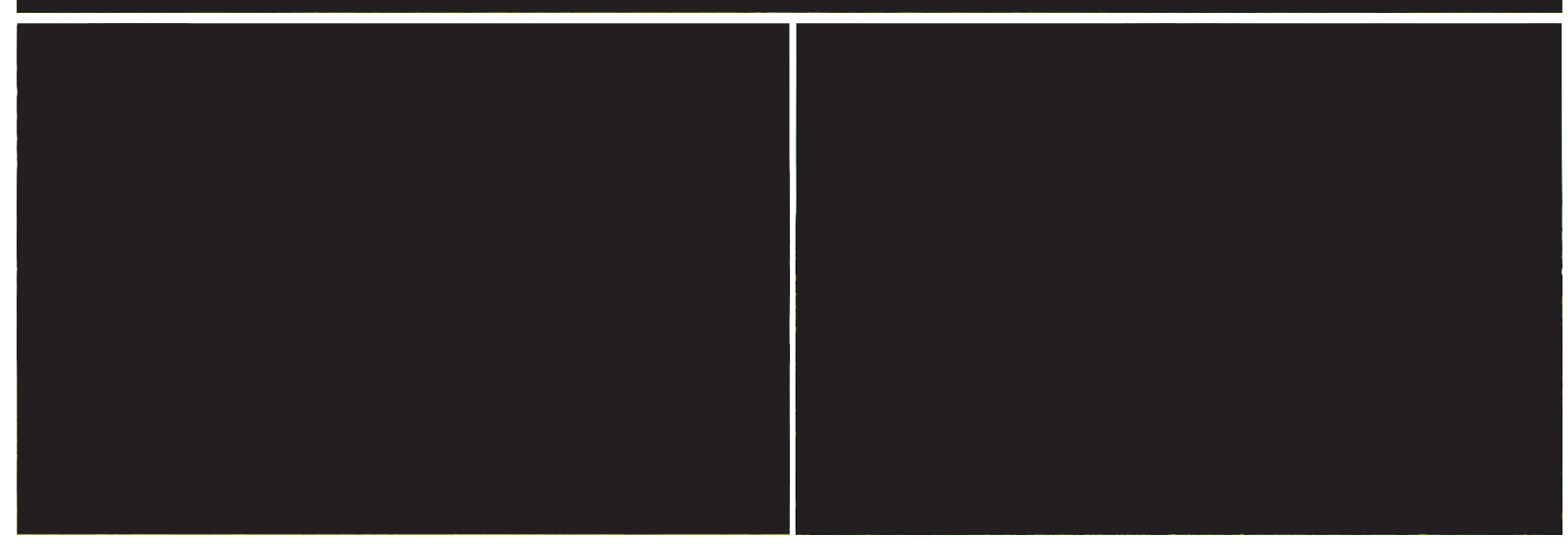

Figure 4.2. The Park, by Smuts-Kennedy, S., Tairoa, T.

Figure 4.3. Victoria Park Meadow, by Smuts-Kennedy, S., Tairoa, T. 


\section{The Park - Auckland Community Project Sarah Smuts-Kennedy \& Taarati Taiaroa Auckland, New Zealand}

Design Features

In Auckland, Sarah Smuts-Kennedy and Taarati Taiaroa have created The Park, an installation of beehives and what they dub "pasture paintings" across the inner city. In Victoria Park, off Beaumont Street, six beehives have been set out in a changing hexagonal planting of bee food.

\section{Purpose}

Expectations of interaction within a park are challenged, making us consider our relationship with nature and how we treat it in a city setting. The artists see the space of the artwork as mapped by the flight paths of the bees themselves, and community participation. "Pasture paintings" have been created on roadside berms in the inner city to provide food for the bees, their designed shapes hot-steamed into the grass.
Communication Design Principles

This New Zealand art project shows some of the many ways information can be portrayed and experienced by the public. The visual display of these working beehives relates to contemporary communication design principles such as the practise of Design for the Education of the Public and Design for Information. The visual aesthetic and central placement of the design poetically connects plant sources with inner city honey bee populations and provides an example of an ideal urban bee habitat. Displayed signs and the physical nature of the installation enable public audiences to be confronted with an experience to show how we can integrate bees into our communities. Communication strategies are employed in this project through its specific urban location that clearly demonstrates New Zealand's bee culture and local practises that have the ability to physically and visually transform our environments.

Reference

Smuts-Kennedy, S. \& Taiaroa, T. (2014). The Park, POP Projects. Retrieved from http://www. pop.org.nz/projects/thepark 


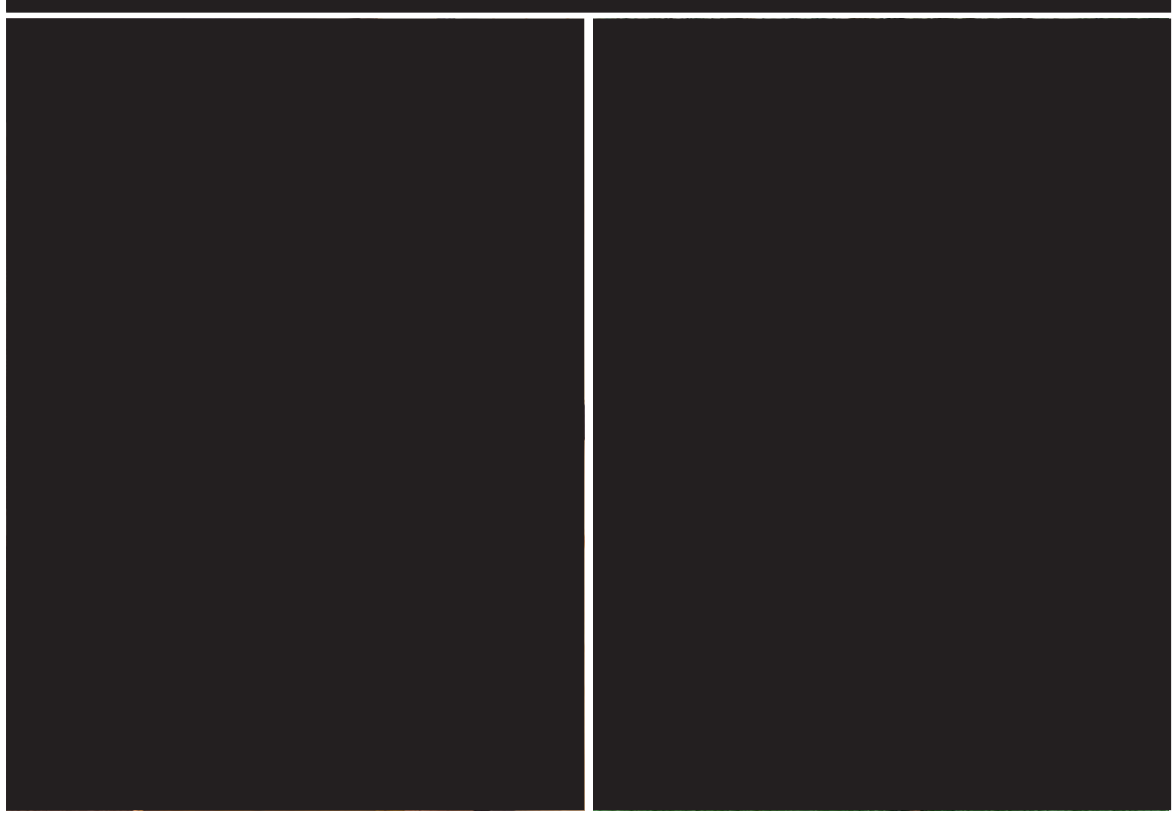




\section{Micro Architecture for Bees Honey Factory \& Francesco Faccin Milan, Italy}

Design Features

This wooden beehive by Italian designer Francesco Faccin has a 4.5-metre chimney to keep its inhabitants away from children and vandals. Currently installed in the garden of the Triennale design museum in Milan, the Honey Factory houses a traditional beehive and all the equipment needed to process the sweet liquid the bees produce from nectar. At the main Milan Expo site, the British pavilion is shaped to reference the honeycomb structures found in beehives, and presents visitors with information about the lifecycle of bees and their role in pollinating food crops. Faccin designed the structure to protect the hive from bad weather, and to keep it at a constant temperature with optimal ventilation. "It can be considered as a real micro architecture that leaves a mark in the urban landscape where it's placed," he said.

\section{Purpose}

The project also aims to promote the idea of urban beekeeping and provide information about its environmental benefits. A door in the back features metal-mesh panels and the front has a thin window, so passers-by can watch the bees and the beekeeper at work inside. "Honey Factory will play a role of educating and allowing citizens to approach a complex and fascinating world of insects, observing them, listening to the bee buzz," said Faccin.

"Through a processing of materials, bees create a rich, comprehensive and sustainable food without reworking, comes directly from the producer to the consumer."
There has been a recent push from the design community to increase efforts to protect the welfare of bees and encourage them to live in cities. Snøhetta installed a series of wooden hives in Oslo, while Bettina Madita Böhm designed a cylindrical concrete beehive for urban beekeepers to use on rooftops. For his project, Faccin worked with beekeeper Mauro Veca to design an optimal habitat for the bees. "I was able to work starting from accurate and reliable information, which is a key condition for an experimental project but scientifically correct," the designer said.

\section{Communication Design Principles}

The visual display of a working beehive in this project relates to contemporary communication design principles such as the practise of Design for the Education of the Public and Design for Information. All external factors of habitat placement, environment and human interactions have been considered by the designer in order to facilitate a positive learning experience for the public. The format and viewing components of the design enables a visual insight into a regular beehive, exhibited in a protective casing to ensure no harm. These visual and informative design features exhibit communication principles as well as providing a different informative experience, unique and dedicated to its specific location.

\section{Reference}

Honey Factory is "micro architecture" for bees. (2015, July 1). Dezeen. Retrieved September 7 , 2016, from http://www. dezeen.com/2015/07/01/ francesco-faccin-honey-factory-urban-beehivebees-triennale-museum-milan-expo-2015/ 


\section{'The Hive' \\ Wolfgang Buttress \\ London, England}

\section{Design Features}

The Hive was inspired by scientific research into the health of honeybees. Installed at Kew Gardens in 2016, the Hive is a unique, multisensory experience designed to highlight the extraordinary life of bees. It is some $17 \mathrm{~m}$ square and is set in a wildflower meadow where the visitor can explore and reflect on the complex relationship between bees and their foodsource. It is the design of UK based artist Wolfgang Buttress, originally created for the UK Pavilion at the 2015 Milan Expo. It is 17 metres tall, is constructed from 170,000 aluminium parts and 1,000 LED lights and took four months to construct.

The Hive is an immersive sound and visual experience. The lights and sounds inside the Hive are triggered by bee activity in a real beehive at Kew. The intensity of the sounds and light change constantly, echoing that of the real beehive. It is a visual symbol of the bees' role in feeding the planet and the challenges facing bees today.

\section{Purpose}

The Hive aims to highlight the importance of the honey bee in feeding humanity, and the challenges facing the species due to climate change, pesticides, and lack of biodiversity - a message which makes it a perfect fit for its new home (after the exhibition in Milan) in a densely populated city such as London. "The purpose of 'The Hive'," says Director of Royal Botanical Gardens, Kew Richard Deverell, "is to tell the story of the role of bees in pollinating crop plants and therefore feeding the planet. I thought that was perfect for Kew because we want to bring alive why plants matter, and clearly one of the most important things that plants do, is to feed humanity."

\section{Communication Design Principles}

This project demonstrates key communication design principles through the physical interactivity of the sculpture in a public space. This targets the practice of Design for the Education of the Public in its compelling nature and large scale simulations of a beehive. The design features target information, persuasion and education of the public and observations are intensified by the enlargement and upscaling of an ordinary beehive. Through this change in scale a different perspective and therefore understanding is influenced by the designer, with other connections to the environment and the concept of climate change being its primary purpose. This immerses people physically in a beehive simulation through sound, light and visuals, using sensorial communicators, similar to visual outputs and experiences encouraged in communication design.

\section{References}

1. Kew Royal Botanic Gardens. (n.d.). The Hive. Retrieved April 25, 2017, from http://www.kew. org/kew-gardens/attractions/the-hive 2. Chalmer, S. (2016). "The Hive" Sound and light installation controlled by bees. Retrieved from http://edition.cnn.com/2016/06/20/arts/thehive-wolfgang-buttress/ 


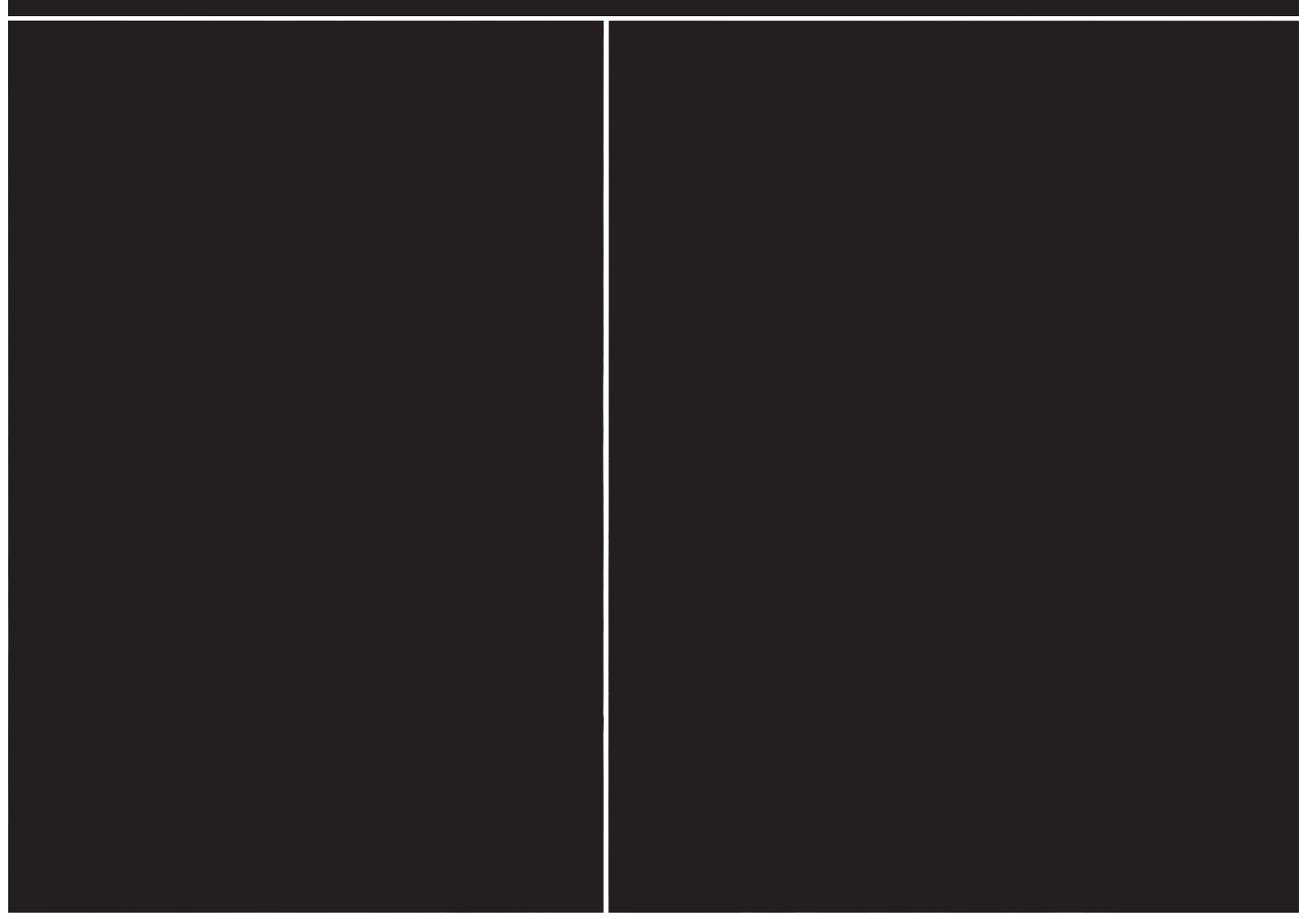




\section{No Vertical Song \& Nature Study Anne Noble Wellington, New Zealand}

\section{No Vertical Song}

A result of a newfound passion for beekeeping, Noble has become "preoccupied with learning about bees and understanding the hive, how it functions and its significance for the larger ecosystem that we are a part of." Her most recent photographic project Song Sting Swarm began in 2013 with an exhibition at Two Rooms. No Vertical Song is the latest installment, showcasing 15 photographs of dead bees, called the Dead Bee Portraits. These works are installed as if populating an imaginary museum of the bee, in a time when the bee no longer exists. The artist's concern with the worldwide decline of the honeybee results in an exhibition that is a haunting and elegiac reminder of the importance of our relationship to the natural world.

Using a microscope to function as a camera rather than a scientific instrument, Noble fuses art and science to illuminate an issue she cares about deeply. In order to align both aesthetic and scientific modes of observation and representation, she invented her own microscopic imaging tools and also worked alongside scientists using advanced electronic scanning machines. The portraits were made with a scanning electron microscope - an imagemaking process employing an electron beam stimulated by the element gold. The resulting works involve the use of light from both the visible and invisible spectrums to create images that seek to encourage a connection to animals, insects and ecosystems.

\section{Nature Study}

While concerns about the decline of the honeybee are at the heart of Noble's project, this exhibition (Nature Study) looks at how we perceive and understand the natural world. Drawing on the strategies of science and more specifically morphology (the study of the form and structure of organisms), Noble uses specimen slides, her own homemade microscope and electronic scanning microscopes to bring us intensely close-up photographs of bee anatomy.
The over-scale images of bee wings titled "Eidolon" (a spirit image or phantom in ancient Greek mythology) suggest something at once essential, ethereal, even angelic. Such images are described at being included to "operate within the exhibition to steer us beyond a rationalist, scientific approach to knowledge to something broader". With scientists struggling to understand, contain and manage colony collapse and the huge economic ramifications for crop pollination, Nature Study evokes a sense of alternative frameworks embodied in the values of the nature study movement and the more contemporary understanding of human impact on the ecosystem.

\section{Communication Design Principles}

Noble uses photographic techniques and imagery to convey a sense of relationships with bees and to illustrate other perspectives by using microscopic camera lenses to intensify the bee's form and features. Imagery and photographic concepts are main considerations in communication design, showing storytelling capabilities through visual outputs. The storytelling aspect and descriptions of Noble's exhibitions imbues bees with a living persona and relates them to human characteristics and functions in an anthropomorphic way, whilst enabling a general visual education of the bee itself. These contemporary techniques speak to the different types of media communication designs which can be exhibited and aid in information reaching a variety of public audiences. The main communication practices exhibited are Design for Information and Design for the Education of the Public through varieties of scale and content. By placing these pieces in art galleries, any wider context is removed, so the focus is purely on the physical form and visual characteristics of the bees.

\section{References}

1. No Vertical Song | Exhibition | Two Rooms.

(2015). Retrieved from http://tworooms.co.nz/

exhibition/anne-noble/

2. Nature Study | Exhibition | Bartley and

Company Art. (2015). Retrieved from

http://www.bartleyandcompanyart.co.nz/

exhibition,Anne_Noble, 276 


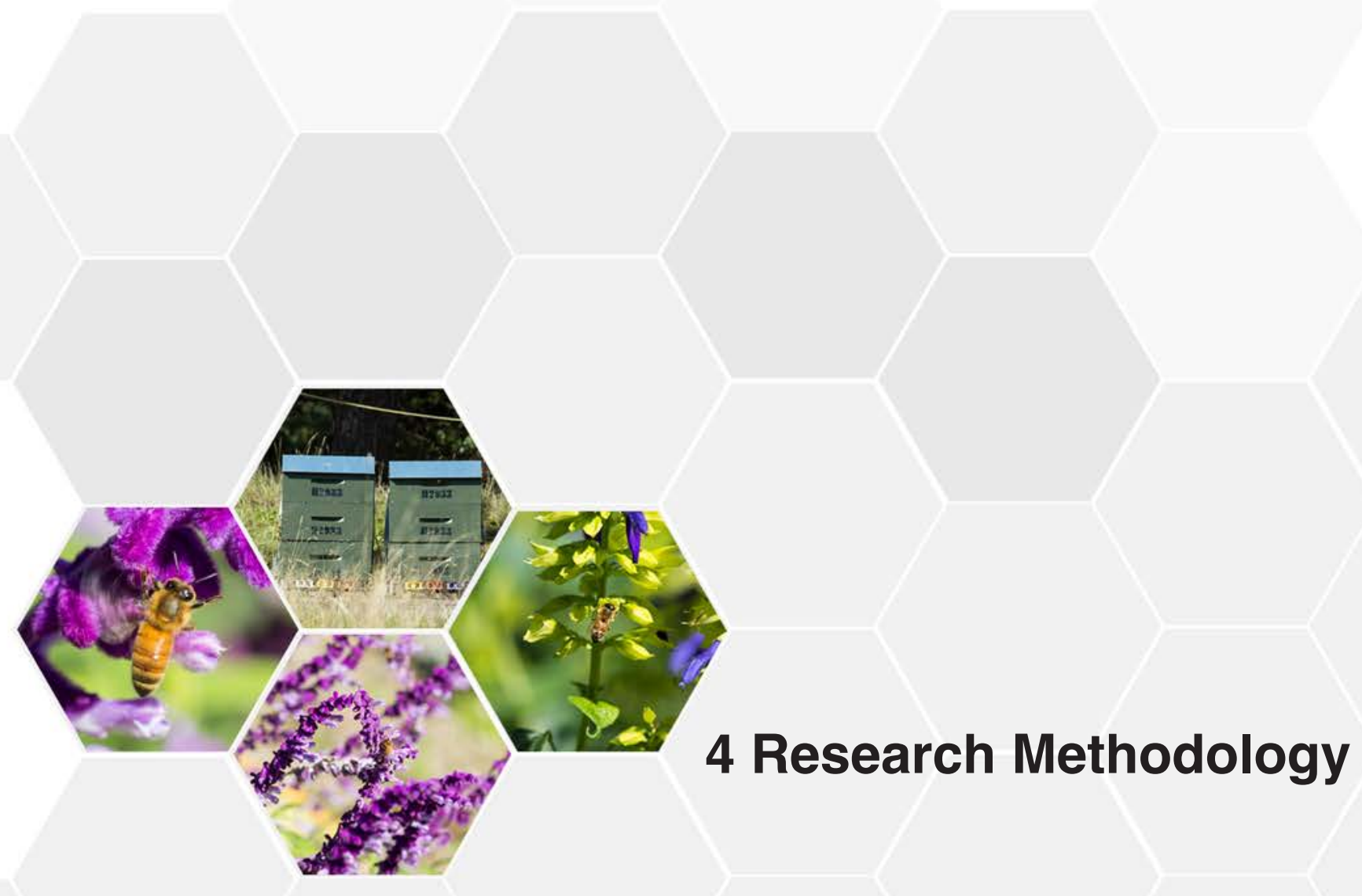




\section{Introduction}

This chapter will identify appropriate research methods to be used throughout the research and design phase of my thesis. Initially, the literature review revealed three key themes that serve as a framework for investigation within this research: sustainable environments; honey bee populations; and the potential value of communication design to promote awareness and to invite action. The preliminary research about design identified the use of design research, visual research with the integration of a human-centered design direction. The design development process is led by qualitative data collection and discussions supported by communication design principles. The use of communication design principles will complete the criteria for the final design prototype, encompassing all design, visual and humancentred methods. Finally, the research through design phase includes iterative designs and feedback from key stakeholders, in order to form a cohesive output that accurately portrays Wellington's honeybee populations.

As discussed in the literature review, there is growing concern for the well-being and caretaking of Wellington's bee species, emphasising the need for them in our ecological system. More empathic relationships between people and bees would encourage positive future interactions for bee species' development and wellbeing. Social and cultural issues are relevant to the sustainability and conservation of current and future bee populations in Wellington, and these methods and approaches include the visualisation of current climates and the impacts it may have on interactions in the future. The communicative design techniques discussed within my thesis focus on the visualisation of information that has the potential to inform positive interactions with existing bee populations. My project locates and visualises the perspective of stakeholders within this issue to address and encourage suitable conservation information and techniques. Online platforms can provide a larger scaled visual insight into bee species and colourful interactions among Wellington's natural environment. The value of communication design visuals and targeted material addresses both the education of the public and designing a tool for education itself. The technological aspect and media platforms target the interactive nature of information and the accessibility of content to both physical and virtual Wellington audiences. By mapping the physical placement of hives within public spaces, this encourages a communal understanding and awareness between human residents and local bee populations. The website and social media platforms are designed to help viewers understand and appreciate the validity of bees in Wellington and increase visual stories of interactions and positive outcomes of keeping bees.

\section{Research About Design Design Research}

Sullivan (2010) suggests; "the promise of change that comes from the imagination takes shape in the things we create, through what we make and experience, or from what we come to see and know through the experience of someone else. Using the visual arts (and design) to turn questions into understanding that give rise to more questions not only describes an aesthetic process of self-realization, but it also describes a process of research" (p.121). The practice of art and design research is described by Mäkelä (2006) as "a dynamic relationship between the research context, the question, the method and the audience. Variations in any of these affects the appropriateness of the chosen method" (p.16). There is no preference or superior set methods. As Mäkelä suggests; "research in art and design is conceived of as being interpretational and pluralistic in nature" (p. 16). Design research author Nigel Cross (1994:3) defines design methods as "any procedures, techniques, aids or tools for designing that 'attempt to bring rational procedures into the design process" (Cross, cited by Nemeth, 2004, p. 99). Nemeth (2004) believes that both research and design methods combine to interpret a collection of new knowledge and the conception and modelling of speculations about how to solve problems. Not only does design research enable an understanding of the end consumer, but "some have used the expression 'empathetic design' to express a more ambitious goal of seeing a situation internally; that is, as potential consumers will see it" (Laurel, 2003, p.39). Laurel (2003) recognises that through the capacities of design research we have the ability to imagine, invent and iterate our way out of a crisis, proving that design is not only a profession by a way of life. Evidently the enabling of various research perspectives fosters an expression of new knowledge with the potential for design to construct solutions and bring rationality into material form. 


\section{Human-centred Design}

In the context of design research and the multitude of complexities it addresses, an initial understanding of the end user is imperative for human-centred design. Rouse (2007) explains that the process of human-centered design considers that "concerns, values and perceptions of all stakeholders in a design effort are considered and balanced" (p.5). Through Rouse's design ideas, the primary objectives within human centered design drive the designers' thinking to enhance human abilities, overcome human limitations, and foster human acceptance. The stakeholder's preferences and concerns are considered, understood and cultivated during the design process. Kleinsmann (2013) also agrees that the designers' purpose is to identify and correspond with primary stakeholders as a means to better understand how user research drives design decisions and itself provides an understanding of end users. In order to focus on the design needs of the end users and stakeholders, it is important to guide the design process through a criteria that influences the portrayal of information, the intended target audience and the use of different media types. The final stage of the design process, described by Bartel and Garud (2009), reflects the skill communication of the designer, where a narrative must demand and connect to the needs and questions of the audience. Agreeing with this process, Kleinsmann concludes that successes between the designer and consumer lie in the communication and interaction of all concerned parties and the way in which this dialog shapes the meaning of particular pieces of information.

\section{Visual Research}

The meaning and communication of visual mediums (photography and graphic design) in my project is important as these will represent key insights into current concerns about Wellington's honey bee populations. Visual research is defined as a study of images, forms and objects from both visual and material culture. Muratovski (2016) believes the ability to critically examine the effects that images and objects have on people's lives is particularly important for all designers, regardless of their disciplinary focus. In my project, the intention is to visually enlighten and improve the audience's visual perceptions of bee populations, creating insight into their individual lives and how they function from day to day, just as we acquire an understanding of a human population. This insight is carefully photographed at a larger scale that evokes the way bees view and navigate their surroundings. This provides a visual contrast to how humans view the world and creates an insight into the role we can have to help and understand such a vital species. The essential aim is to trigger empathic relationships and to stimulate an awareness of that local surroundings and ecosystems are not "ours" but are shared. Muratovski supports this by asserting that certain applications of visual content require a consideration for the intended audience, accessibility, and the embedding into particular contexts that "involves thinking about how the viewers and the users are positioned in relation to the image, the form, or the object" (p.162). Here, a visual platform across online and social media channels targets a younger, technology-efficient audience by creating a place to collect and view common stories and encounters. In order for the visual communication of this design to resonate with users, ideas, customs, and behaviours of a particular person or community, I will provide the ability to become visually embedded in a wider cultural and environmental context. It is important for me as a designer to show that every individual action or interest can lead, cumulatively, to a collection of positive actions, the sum of which is greater than its parts, including having the ability to extend beyond a Wellington context. 


\section{Qualitative Data Collection}

Recruitment of Participants, Semi-Structured Interviews,

Stakeholder Perspectives \& Design Suggestions

The recruitment of participants took place through local community events, recommendations and online research with relevant stakeholders and organisations. Through industry suggestions from the Wellington City Council, key stakeholders were identified and contacted with a proposal to be included in the design phase of my thesis. The key respondents included the Head of Biology at Victoria University, the Wellington Beekeepers Association, the Beeple Community project and the Wellington Botanic Gardens. The specialities of expert individuals participating include academics, beekeepers, and local community and conservation advisors with leading roles in research and projects concerning the education and wellbeing of Wellington's current and future bee populations. These insights provided a detailed depiction of the current state of bees in Wellington, as well as providing expertknowledge of areas in need, specifically targeting the future management and identification of barriers to growing bee populations. In total, there will be one representative from all four organisations, enabling the capture of a holistic view, inclusive of academic research, current education and community bee projects/practices. In the field of communication design, "it is more common to suggest that visual designers "gain information from the public" (Frascara, 2004) through formative interviews or usability tests of prototypes, butrecommendations focus primarily on the aesthetics of representation" (Frascara cited by Kleinsmann, 2013, p.145). Content selection and communication design strategies influence the visual elements of the final output, staying true to information and recommendations made by the expert participants.

Qualitative research is defined by Creswell (2014) as "an approach from exploring and understanding the meaning individuals or groups ascribe to a social or human problem" (p.183). As determined by Creswell, the reason for designers to conduct such research is "because we need a complex, detailed understanding of an issue" (p.183). This approach is used in my thesis in the incorporation of all key stakeholders in Wellington's bee industry to account for all current and future concerns for honey bee survival. Creswell proposes a holistic account where researchers try to develop a complex picture of the problem of issue under study. This involves reporting multiple perspectives, identifying the many factors involved in a picture that emerges. Laurel (2003), too, values qualitative data collection because "individual interviews are ideal for learning exactly how each person feels and thinks about a topic or design, without concern for the influence of others" (p.25). The variety in the stakeholder accounts acquired here comes from their current position in the bee industry, with a range of information and answers arising from academic, handson and active participant information and bee encounters. Evolving from this idea, Nemeth (2004) considers that qualitative methods such as in-depth interviews are "most useful and powerful when they are used to discover how the respondent sees the world" (p.254). In summary, Creswell believes the entire qualitative research process "involves emerging questions and procedures, data typically collected in the participant's setting, data analysis inductively building from particulars to general themes, and the researcher making interpretations of the meaning of data" (p.183). The commonalities in each interview provide a realistic approach to designing for a purpose, as the design aims to fill current gaps in education and provide communicative tools that can be applied to future bee ecology contexts.

To appropriately analyse the data and interpretations of the honey bee industry collected from the semi-structured interviews, a thematic analysis has been used. Guest, MacQueen \& Namey (2012) believe that this design research process "means that qualitative researchers study things in their natural settings, attempting to make sense of, or to interpret, phenomena in terms of the meaning people bring to them" (p.4). The process of a thematic analysis is defined by them as "textual data generated from in-depth interviews and focus groups - which are often transcribed verbatim from audio recordings - and, to a lesser degree, participant observations notes" (p.10). It is required that the researcher be more involved and interprets explicit ideas from the data, and thereby describes and identifies key themes. Saldana (2009) defines a theme as a "phrase or sentence that identifies what a unit of data is about and/or what it means" (Ryan \& Bernard, 2003, p.65). Ryan and Bernard provide tips to identify themes through the recognition of: repetition, metaphors and analogies, transitions and silent/ missing data (2003, p.66). This process is ideally the most suitable as it deconstructs transcripts of interviews in order to identify recurring ideas and main concepts to focus the design output. 


\title{
Application of Methodologies to Design Development
}

\author{
Communication Design
}

The communication design strategy structuring my thesis uses disciplinary approaches and tools to aid in producing a concept inclusive of communication design principles. I chose this particular design practice for my methodology because the main objective of the design is to "influence public good" (Frascara, 2004, p.35), pursuing hidden dimensions of our social and environmental communities which require communal solutions that, in turn, call for persuasion to a way of thinking. Resonating with my beliefs as a designer, Frascara (1997) considers that "it is necessary for designers to recognize the needs of the social and physical environment within which they work and to which they contribute, and to take conscious steps to define the future direction for their profession" (p.19). Similarly, one of the modern design applications of communication theory was propounded in 1983 by Patrick O. Marsh, who instructed that "the communication design model consists of a source, a message and a receiver" ( $p . x v i-x v i i)$, and he used a synthesis of several diverse disciplines, theories and points of view to devise a systematic process.

Communication design was first described in Jorge Frascara's 1997 work, with the initial principle that to "design is to invent, to project, to program, to coordinate a long list of human and technical factors, to translate the invisible into the visible, and to communicate" (p. 2). Communication design is defined by Frascara (2004) as "the process of conceiving, programming, projecting, and realizing visual communications that are usually produced through industrial means and are aimed at broadcasting specific messages to specific sectors of the public" (p.2). Adhering to these notions, the intent of my thesis is to impress a positive focus onto public consciousness, attitudes, behaviours and relationships concerning Wellington's bee populations. The main communication principles incorporated in this project are; Design for Education and Design for the Education of the Public. In Design for Education, the work of visual communication designers moves between informative and persuasive avenues. Persuasive communications intend to affect the behaviour of the public, alongside educational messages that intend to contribute to personal development (Frascara, 2004, p.152). Relating to design for the education of the public, objectives relate to methodical terms of all three types of communication design: information, persuasion, and education. The development of these principles forms a visual design strategy to combine multiple avenues of information and intended audiences (2004, p.158). Frascara appropriately cautions, however, that education cannot rely solely on the passing of information, and that educational materials must engage participants and communicate different types of visual learning and interactions, just as Socratic teaching seeks to impart knowledge in a sustained way.

Communication design principles and visual design techniques help to establish the design criteria regarding the value and purpose of decorative elements and content on the website. Communication images aim to bring a dimensional output that supports the perception and meaning of information on multiple platforms. Significance and relevance are major determinants for calling attention, "as communication, in turn, does not end with attracting attention. Essentially, it involves making information understandable, usable, interesting, and, if possible, pleasing" (Frascara, 2004, p.61). These are definite considerations in assessing the clarity and purpose of images, in communication design. The inclusion of images, per se, is imperative on educational websites and social media platforms, and "must be visually strong, [and] highly cohesive" (Frascara, 2004, p.59), and must be tailored to move from being captured in a physical environment to being uploaded into a virtual one. The reality of such images must portray the context surrounding the bees/beehives and ecology, and visually convey an enriched human encounter. The images must be strong enough to emerge clearly and become separate from the online context. This technique ensures every visual aid tells its own story and evokes different reactions from the viewing, alone, of the website's written content.

This website platform, supported by the communication design principles for media design and interface interaction, allows for a contemporary technological approach to be recognised and influential. Frascara (2004) suggests that interaction is central to communication, for stimuli to become information, one has to actively interpret, through a variety of actions - to live is to interact. Essentially, every possible physical and virtual "interaction is our human way of dealing with things and with information" (p.173). 
The relationship between human interaction, the processing of information and understanding is facilitated through presenting information with the ability to manipulate its content across media platforms. In terms of both the profile of the universe where one is and one's location within it, Frascara asserts that "the design of interfaces must respond to the need - that sense of "where am I?" (p.174). Rationalising and relating the content to a Wellington context allows audiences an experience and visualization of a time and place - relevant both to them and to the honey bees and beehive locations. A major concept in terms of design thinking that comes to play is the notion of usability: ease of use, ease of access and ability to be found (Frascara, 2004, p.174). At the same time, Nemeth (2004) rationalises design thinking and human centered design through a technological approach, enabling everyday experiences "to extend human abilities and to overcome human limits" (p.3). The marrying of usability with a technological approach requires discipline in design, given that the object of the website is to facilitate an understanding of a different existence - that of the bee species. These design ideas are met by the crossing of physical and virtual interactions encouraging relationships among humans, organisations, the environment and structures. Nemeth puts an emphasis on the need for these socio-technical system designs to fill the inevitable void between our physical and virtual relationships, considering the effects on all parties involved.

For my design output I have chosen to incorporate multiple social media channels extending from the communication design of the website, with the aim of expanding the accessibility of information and encouraging interaction. Zhang \& Lin (2015) define social media as a group of internet-based applications which carry the content and present it in various formats. The evolution of social media has seen an increased flexibility and visibility of content and changes in the way in which individuals and organisations interact. There is a significant amount of social and network value to users through social media, as users comment, review and share information online. Zhang \& Lin believe that social media enables consumers to initiate and gain control in the communication process due to its enhanced interactivity features. The internet has enabled specifically contemporary ways to engage with consumers in new and meaningful ways whilst maintaining an instant and constant dialogue. Social media allows every user to "enjoy the equal opportunity to create, contribute and disseminate messages, such user-centered content has unprecedentedly enriched communication, both in its content and in format" (Zhang \& Lin, 2015, p.671). Reiterating this perspective, Dolan (2017) has redefined social media behaviours to include a particular focus on the consumer's engagement through creation, contribution and the consumption of content within a social network. From Dolan's research, three main objectives are addressed demonstrating that communication happens through four main types of social media content: informational, entertaining, relational and remunerative. Perhaps conclusively, this is followed by Dolan's indication that the most common type of post used by brands is photos.

Due to the diverse existence of social media interfaces, the two platforms that I have chosen for my thesis are Facebook and Instagram. Lipshultz (2015) records that Facebook is the most popular platform and takes the biggest market share, allowing users to utilise various features designed for interpersonal communication, whereas Instagram allows audiences to piece together images into a larger narrative of storytelling. These channels, especially Instagram, have a very visual and interaction approach with capabilities targeted at younger consumers. Lipschultz concludes that sharing information through social media apps has become an integral part in the way people in general - and particularly youth - choose to express themselves online. This reality has led me to design the website for a target market of students and young professionals in Wellingtons, as this age demographic frequently uses such online platforms. In my thesis, the interactivity of social media generates a sense of presence and encourages people to use it with friends and communities, engaging audiences through the medium of a social community interaction. Social media activities and the sharing of images is encouraged and connected to via a QR code at public beehive sites in Wellington, aiming to connect users with both the physical and the virtual environments. The website allows for all images and locations to be collated, enabling the public easy online access to Wellington community bee stories and experiences. The bee, in this sense, acts as an engagement strategy for sharing images through social media, visually encouraging audiences to take part in the spread of knowledge and interest in our environment and ecosystems. 


\title{
Research Through Design
}

\author{
Iterative Design Process, Concept Testing, Stakeholder Feedback
}

The design testing component will include an iterative design process, concept testing and feedback from key stakeholders - working from a design criteria and framework for evaluation constructed through the communication design principles. An overall iterative concept testing approach will allow the visual and content design to be presented consistently to the key stakeholders, as indicated in the semistructured interviews. Reflecting on the literature review and the content from the semi-structured interviews, the design will then guide and produce knowledge by research through design. Grainger recognises that "fully implementing a UCD [user centred design] approach requires an iterative design process involving prototype usability testing and an evaluation of tool efficacy from the user's perspective" (2016, p.306). Situating the design among potential end users, in the iterative design process helps the success of visual design studies as "researchers try to become familiar with the end user's realworld environment by observing them in their workplace" (Grainger, Mao, \& Buytaert, 2016, p.306). This then leads on to the idea that concept testing "allows you to take a few ideas out to your audience of early adopters and put them to task. One of the benefits of doing a concept test quantitatively is that a clear winner emerges from a few concepts" (Laurel, 2003, p.65). The process of concept testing includes guiding respondents with some kind of stimuli - words, pictures, or a rough prototype. This process is most useful at this early point for assessing how unique and relevant the concepts are and, essentially, whether the ideas are suitable for the intended audience (Laurel, 2003, p.65). During the process of validation and feedback from stakeholders, further iterative design prototypes may be developed and variations of the design may be presented to refine the output, leadings to better and more accurate results. Recommendations from interviewees will be taken into account and considered because the goal of qualitative studies in design is to reduce, rather than to add, complexity. The interaction of qualitative studies within an entire research process is a dance of expansion and contraction of possibilities, but always moving towards an optimised design (Laurel, 2003, p.68).

\section{Conclusion}

The ability for design to address social and environmental issues asserts its validity as a professional and unique way of thinking. The complexity of a design needs to be defined by its process, intended content and real world application. Throughout my design process, key research and design contributions are asserted through research about design itself and the iterative process of research through design. Experiencing and creating through communication design principles allows for specific principles to be applied to the process of human centered design. The use of qualitative research locates the observer in the world - "it consists of a set of interpretive, material practices that makes the world visible. These practices transform the world" (Guest, MacQueen \& Namey, 2012, p.4). Input and data from interviewed experts brings richness to the type of information needed to focus a relevant design direction. Finally, understanding both the design criteria and target audience enables valuable feedback to guide the iterative design process and to test the correct interpretation of information and images. The purpose of my output is to formulate a visual interpretation of information through communication design principles, to inform audiences about current conservation and sustainable practices necessary in Wellington. The honey bee is a key influencer in all of our ecosystems and communities. For the future stability of honey bee environments, and their health and wellbeing, individuals need to understand that their existence is vital and that it is necessary to be a part of influencing a sustainable change. The perception of bees and their value (to themselves, the environment and people) needs to be shaped through education, in order to realise what kind of current role humans need to play, instead of waiting for the inevitable decline of bee colonies to awaken a sense of urgency in their survival. By then, it may be too late. 


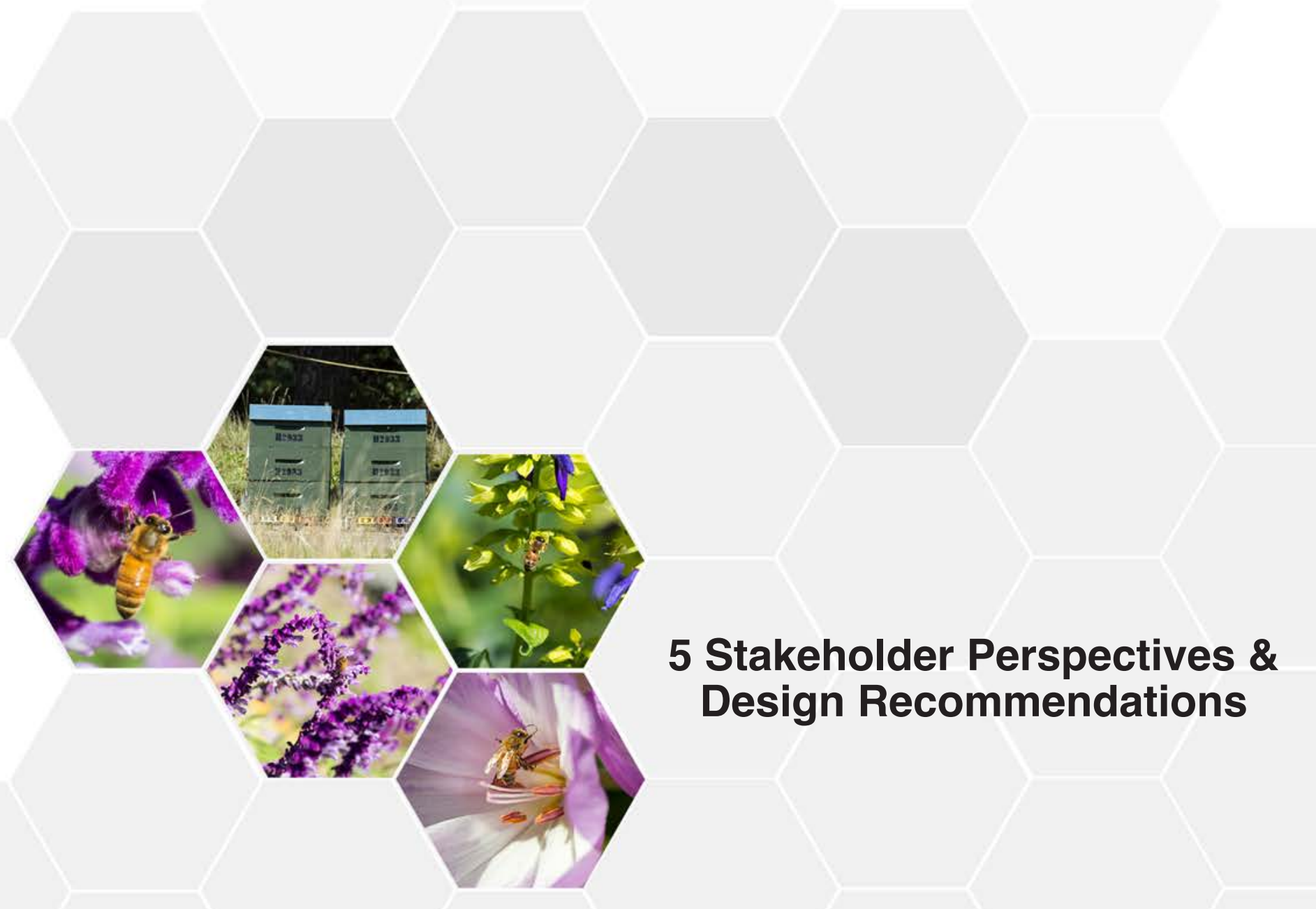




\section{Introduction}

This chapter will discuss perspectives from the local bee community in Wellington, and suggest how they can be incorporated into a set of design recommendations for a communication design strategy. The questions designed for the semistructured interviews were aimed at gaining a clearer understanding of honey bees themselves, their interactions with humans, and the education and promotion that surrounds them. Questions also addressed the barriers or negative impacts on honey bees currently apparent in Wellington, involving local organisations, businesses, residents and environments. The particular people involved in these interviews had various levels and areas of expertise, education, and practices in the honey bee industry, ranging from academics to beekeepers themselves. These individuals included representatives from; Victoria University of Wellington (VUW), Wellington Botanic Gardens, the Wellington Beekeepers Association (WBA) and the Beeple Honey Collective. The Beeple Honey Collective is unique in its approach to bees, because the organisation initiates various types of educational projects in Wellington, with honey bees and beehives being a part of a larger community focus. A variety of perspectives were sought through interview questions, in order to play to the strengths of organisational experience, locality and current understanding of honey bee populations. A conversational approach was used, so that both amateur and expert opinions and information could strengthen my understanding of the current state of Wellington bee ecologies and beekeeping. Commonalities among responses have been recognised as main themes to address in my design, with specific topics among these providing visualisations and content to cover. The main themes include Wellington's honey bee culture, management, education, and environment, all influencing communication design recommendations and a criteria for the design output.

\section{Culture}

The current culture of honey bees in Wellington, as indicated by the VUW expert, acknowledges that "there is an awful lot of public support around honey bees as it is, and it's growing." Described by the WBA's spokesperson, Wellington "is a good place for beekeeping because of all the gardens here". Supporting this, the Botanic Garden's representative explains their importance as "an essential ecosystem service for us". The benefits of bees in Wellington is recognised through all expert opinions as the bees' function and location signify their relevance to our culture and the beneficial effects that they have on our ecosystems. The key narratives in the cultural identity of New Zealand bees include positive indicia, such as the Manuka honey industry and bees as pollinators of native flora, as well as negative indicia, such as the fear of bees and of being stung, and obstacles to understanding, such as confusion between wasps, honey bees and bumblebees. These contributing factors were raised as concerns by stakeholders, especially when misinterpreted or viewed adversely by the public. Interviewees agreed that education is essential to address fears and behaviours towards our bee populations. Healthy relationships with our bees should highlight the mistreatment (such as stress from over-interference with hives) and potential overpopulation of Manuka bees, create an awareness of the honey bees' pollination role in New Zealand and educate people as to the visual differences/stinging actions of relevant insect species. 
The importance of the honey is reflected in the large quantities of Manuka products exported from New Zealand. A 2016 Stuff news article by Gerard Hutching refers to statistics showing that "currently, total honey exports are worth \$242 million a year, of which Manuka makes up about 80 per cent. The Government and a consortium of companies have a target to reach $\$ 1.2$ billion for Manuka honey alone by 2028." The primary focus of public beekeeping education, as articulated by the Botanic Garden and Beeple interviewees, should be to encourage the pollination role of honey bees and the benefits they provide to our native plants and crops. The amount of exposure the Manuka industry receives, however, overrides the purpose of the honey bee in New Zealand, and forces beehives to produce large amounts of honey even when living conditions are not suitable. It was suggested by the WBA and Beeple representatives that excessive farming and reliance on one crop (Manuka) has meant that if the crop does not flower due to certain conditions, honey cannot be produced, hence the dramatic seasonal variations. The VUW spokesperson indicates that known consequences from this market becoming incredibly popular in New Zealand have been; "a large stocking of bees around the country, and potentially poor management". All representatives voiced their concerns for the Manuka industry, especially Beeple's representative who explained; "there is so much money involved in it that people are mistreating bees, they are letting hives die, they're taking everything and feeding hives sugar in the winter instead of leaving them honey to eat." Further expansion hopes for the Manuka industry include an increase in productivity by 16 -fold, with early signs being promising after just a few years of improving genetics and management. This altering of processes also lends itself to the altering of land; "if such productivity gains can be made, they will transform the farming of marginal, erosion-prone hill country" (Hutching, 2016). The push for productivity in the Manuka industry means larger populations and farming of bees, with more Manuka transformed areas. The Beeple representative believes that the global demand for Manuka products encourages the exploitation of bees, and the altering of their natural processes and rhythms.

Representatives from each organisation all identified, as a concern, that the fear of the honey bee stinging leads to the desire to kill bees. In reality, it is founded on a major misconception, because the bee is docile and only harms people when aggravated. This fear is more common in younger generations, and results from ignorance. As the WBA member suggests, "a lot of it is just visual, a lot of people get easily intimidated by the sight of hives and bees milling around, they don't have to be stung". Confusion between bees and the common wasp and bumblebee species also leads people to see and treat all bees as pests. This common misperception can lead to the mistreatment of bees, potentially resulting in the banishing or removal of them from a certain area. Beeple's representative explains that this fear is disappointing as "we are so dependent on them, it is such a misconception to think that they are somehow dangerous to us - without them we are in danger, it needs to be flipped on its head I think."

A biologist from VUW indicates that, nevertheless, there is a "popular rise in urban beekeeping currently in Wellington". Evidence of these increases are voiced by the WBA spokesperson who advises "We are lucky in Wellington - we have a large group of interested urban beekeepers. In the club, for example, we have 350 and most of those would be keeping bees." Contemporary bee enthusiasts and hobbyists have pushed a positive trend in bringing communities, individuals, and bees together. The Beeple's representative believes that "bees are an amazing reflection of humans... and how we should work together and work as a community." They also describe the Beeple beekeepers as "watchful, loving and passionate" and, through the Beeple Project, "getting people back to community - helping each other out and realising that we are all in this together". Members of the beekeeping community sees themselves as compassionate, nurturing and concerned individuals with the want to benefit personally and altruistically from keeping bees. The realisation for self-sufficiency and sustainability among these beekeeping residents reflects the importance to them of maintaining a healthy relationship with their hives. This illustrates well the crossing of communication between human and more-than-human populations who rely on this reciprocal relationship - we benefit from bees and the bees benefit from us. The WBA and Beeple interviewees believe that no beehives can now exist without human intervention, with both experts voicing their concerns about the capabilities of new beekeepers. 


\section{Management}

The correct management of beehives was a urban environments impose limitations on the main concern of all stakeholders as the practice of beekeeping relies heavily on the education and experience of new beekeepers including; ordinary hive inspections, maintenance, and regular disease prevention. Other external contributing factors include the availability of suitable nectar sources, the positioning of hives in urban areas and the public knowledge and awareness of the bee. The current (and future) dependence of bees on humans means that a lot of preparation and education has to go into the beekeeping industry, making it more crucial for specific information to be readily available.

Without prior beekeeping experience and knowledge, there is a higher risk of the beehive dying out or contracting diseases. In New Zealand, bees suffer from exposure to the varroa mite, the spread of a recently-introduced disease, American Foulbrood (AFB), and encounters with poisonous pesticides. The WBA representative believes "some people still think that they look after themselves pretty much and it doesn't happen that way - it used to 20 years ago. They have to have treatment for the varroa mite on a regular basis." Currently, Beeple's representative believes that it is essential that "we are answerable at some point because I think that it's just carelessness or lack of education around beekeeping is what's going to spread these diseases". There are no prerequisites or requirements to practising as a beekeeper, so the first season of keeping bees is generally the hardest to manage, and uncontrollable factors also influence the survival of beehives. Beeple's representative acknowledges various barriers to growing Wellington's honey bee populations including; food scarcity, lack of seasonal variation, habitat conditions, and overpopulation of humans and hives - all contributing to the well-being, or lack of well-being, of hives. The Botanic Garden spokesperson suggests that foraging capacity of bees as they normally travel up to $5 \mathrm{~km}$ radius. As an example; "the Botanic Garden only has four beehives at the moment but we can add a lot of hives before we reach our maximum in terms of foraging capacity, because of the amount of flowering plants". Yet few urban areas can offer such a range and extent of food. With an increase in urban beekeeping, overpopulation in certain areas can lead to a scarcity in food sources, meaning that it is unable to feed a larger volume of bees. The proximity of hives and habitat spaces must take into account the amount of available land and resources when ensuring that there is a plentiful food supply. The WBA representative supports this by explaining that, for individual beehive sites, "it's generally accepted in an urban environment that no more than four is appropriate, otherwise the bee numbers just get too many, inappropriate for a built up area". The WBA representative explains that "if you are in a city environment such as Wellington, where backyards are very limited and in some cases non existent, it makes it very tricky - so in some cases compromises have to be made. You might not be able to provide the ideal site". There is a general perception across interviewees that bee knowledge is essential for all beekeepers and necessary for the public, as bee encounters are becoming more popular across Wellington. To aid in supporting Wellington's bee practices, the WBA representative suggests that residents should realise that beekeeping is "a sustainable, beneficial hobby for the environment, everybody benefits with bees around." Education could help mitigate unnecessary feelings of fear and aggression, and encourage positive types of interactions and personal instincts. Further, education should lead to better communication among human populations regarding the frequency and location of hives, to minimise the stress to hives caused by over-intensive placement. 


\section{Education}

Various types of education tools were discussed with all interviewees, particularly the types of information distributed by these organisations to Wellington audiences and the platforms they use to communicate. The channels of communication suggested throughout the interviews include online websites, publications, Facebook and Instagram as well as community events such as workshops, trade shows and guest talks. Current types of information are targeted towards each organisation's purpose and role in the bee industry, with more specific bee and beekeeping information being addressed by the VUW and WBA stakeholders. This is due to their extensive knowledge and experience in their academic and beekeeping fields, both having a lengthy dedication to research and the wellbeing of bees. The Botanic Gardens and the Beeple Honey Collective are community and activity based organizations that look into interactions with bees and public experiences, with educational material to support the general knowledge and awareness of bees. The different approaches to sharing information is considered in terms of the topics and ideas these organisations are wanting to communicate. Whether it be academic research, beekeeping education or general bee awareness, all generated information has a purpose and a target audience.

The VUW representative explains that "we give research about bees, in the media occasionally with some stories" combined with "quite a few talks, public talks including bees and native bees". Information about beekeeping is the main focus for the WBA representative as "there is quite a lot to learn, we encourage people to join the club and learn at our club meetings and field days." Supporting these immersive experiences, "there is a bit of just general beekeeping information exchanged on our website between experienced and inexperienced beekeepers - so it's a forum for people wanting to talk or find out about bees." The practice of beekeeping requires a lot of initial education, experience and costs. Because of this, the WBA representative explains that "we don't want everyone to become beekeepers but we would like them to be sympathetic to bees one of the things we do push is the urban trees for bees (online and brochure) which lists all the plants, flowers, shrubs and trees that are good for bees in terms of providing nectar and pollen sources". Another WBA contribution to Wellington communities is this example; "we just had a stand at the Better Home and Living Show last weekend and the main objective was to just raise public awareness of bees, answer any questions people might have - whether they be basic or fairly advanced." Significantly, the WBA representative suggested a specific target market for Wellington; "we could do more about educating the young about beekeeping, I think people are far more aware and interested in not just beekeeping I suppose but self sufficiency."

The Botanic Gardens are focused on providing visual examples for local and international visitors, concerning garden practices and bee education, specifically with the inclusion of their two bee hive locations. The Botanic Garden representative suggests: "I think if we can't get the message out there, then I think what will happen is life without bees." This is why their main focus is to have "big permanent signage made up for the pollinator areas, to explain the hives and everything". The Beeple Honey Collective advise that their forms of education and information exist "through our online avenues, Facebook, Instagram [and] our website. Facebook is really the main driver, we get people joining all the time, it's extraordinary. And if there's a write-up anywhere we suddenly get this massive influx of people joining the Facebook page". Other communication avenues for Beeple are "word of mouth, which is really big for the Beeple project itself, it's really big - people tend to talk about it", along with "breakfast clubs, they are actually going to get our honey and talk about it - we've been donating a kilo into all sorts of little charities and I guess it starts a conversation and that's really what's important". Personal dialogue and online communications suggested by the Beeple representative are accentuated through "other projects happening in here (the Lower Hutt community building) who bring elements of bees, suddenly we start seeing bees in people's consciousness just because they are around it".

As a result of their efforts in communication, these organisations clearly influence the types of information Wellington audiences are exposed to, raising awareness that success relies on the interest and sharing of individuals, followed by a want to be engaged in Wellington's bee community. 


\section{Environment}

The local knowledge and experience of the Wellington Botanic Gardens and the WBA identified various habitat and living conditions for local bee populations as being critical to our understanding and education of future Wellington environments. Influencing factors suggested by these representatives include weather conditions, inner city and suburban land use, local practices of sustainability, self sufficiency and the global impact of climate change. Ideas for human intervention included the planting of flowering plants and the monitoring of hive intensification, and and awareness of how changes in the surrounding climate are affecting beehive survival rates in Wellington.

Ideas of self sufficiency, recycling, and sustainability have started to impact the way people care for their surrounding environments, especially living conditions for future generations. The WBA spokesperson says "many beekeepers are doing it for environmental reasons, they might be doing it for pollination reasons, benefits to the environment - the suburb or the city for example. They just like the idea of doing something for the ecology". Beekeeping is another step towards providing a better climate, particularly for our native ecologies, but also for human benefits and recognising the need for positive human intervention. One of these positive actions is avoiding the use of harmful pesticides and planting excessive amounts of crops, instead of flowering plants. The WBA spokesperson explains, "[as to] sustainability in general, I think everybody understands now more than ever that bees are an essential part of our environment and we have got to do more to look after them".

The knowledge and perception of all interviewees indicates that, in order for bees to perform, they must have the right weather conditions; a warm temperature, majority of daytime sunlight, minimal rain along with shelter from prevailing winds - a frequent problem in Wellington. If these weather conditions worsen or last for a full season, the sub-optimal conditions become detrimental to bee survival. The Beeple representative believes "bees have a huge part to play in education around climate change," as temperatures soar or wetter/colder seasons become more apparent - this lessens the time bees have to function. We've lost touch with our environments and the bees kind of keep us in touch". This climatic change is addressed in Wellington's implementation of the 100 Resilient Cities plan, Sustainable Development Goals (SDGs) and our eco-city focus.

\section{Design Recommendations}

There is a realisation from all participants that, currently, there is a popular interest in Wellington beekeeping practices, as hive and beekeeping numbers continue to rise, specifically in urban areas. The specific ideas that should be addressed in the design relate to the main topics of culture, management, education and the environment. The ideas within each section could be improved upon, grown, or solidified to address the issues and concerns of Wellington's bee health and well being. The cultural aspects include New Zealand's commercial associations within the Manuka industry, the honey bee's function as a pollinator, general fears of being stung, recognising the difference between bees and wasps and an individual and community responsibility. Management issues include the experience of beekeepers, the process of hive inspections and disease monitoring, the availability of food sources, monitoring hive intensification and the knowledge of harmful pesticides. The education section concerns the communication of information in Wellington, types of information being addressed and general bee knowledge. Finally, the environment section includes specific weather and habitat conditions needed for bee survival, and bee practices necessary for sustainable and self sufficient living.

The design ideas derived from the themes discussed can be visualised through communication design principles to adhere to the design criteria structured for the website prototype. The clarity, stimulation and consideration of all cultural, social and ecological concepts in this analysis will visually communicate the importance of the honey bee in Wellington. Design for the Education of the Public is a Communication practice that focuses on the distribution of messages and persuasive information to particular target audiences. The coherency of the topics being addressed in the final prototype needs to be graphically, visually and conceptually appropriate for the target audience and must be applicable to various media platforms. Communication design guidelines propose that the overall purpose of these messages needs to be clear to the audience, and they must relate to a Wellington context. There must be a good relationship between the topic and visual presentation of content, by creating online platforms to raise awareness and educate public audiences. A high aesthetic value has a better chance of being kept active in the minds of the public, which puts a heavy importance on the craft and legibility of information and images. 


\begin{tabular}{|c|c|c|}
\hline RESEARCH THEMES & RESEARCH SUB-THEMES & DESIGN RECOMMENDATIONS \\
\hline CULTURE & $\begin{array}{l}\text { Cultural associations (Manuka - } \\
\text { land \& People) } \\
\text { Honeybee function } \\
\text { (Pollination) } \\
\text { Fears of being stung, aggressive } \\
\text { behaviours } \\
\text { Difference between wasps, } \\
\text { honeybees \& bumblebees } \\
\text { Personal responsibility, } \\
\text { community involvement }\end{array}$ & $\begin{array}{l}\text { The prototype should first } \\
\text { address the current cultural } \\
\text { perception of the honey bee and } \\
\text { visually reinterpret the Manuka } \\
\text { honey industry, fears of being } \\
\text { stung, threatening or aggressive } \\
\text { behaviours associated with } \\
\text { bees, visual differences between } \\
\text { bee species and our personal } \\
\text { responsibilities as positive } \\
\text { influencers. }\end{array}$ \\
\hline MANAGEMENT & $\begin{array}{l}\text { Beekeeper knowledge \& } \\
\text { experience } \\
\text { Disease \& Inspections } \\
\text { Food sources, seasonal } \\
\text { variation (trees for bees) } \\
\text { Pesticides (neonicotinoids) }\end{array}$ & $\begin{array}{l}\text { The management section } \\
\text { identifies that the design should } \\
\text { portray the crucial involvement of } \\
\text { beekeepers, correct practises } \\
\text { e.g. regular disease treatments, } \\
\text { inspections and general hive } \\
\text { maintenance, and an insight into } \\
\text { the level of skill required. The } \\
\text { design should also acknowledge } \\
\text { external food and plant types } \\
\text { that bees prefer, seasonal } \\
\text { flowering variations and } \\
\text { education of harmful practises } \\
\text { like pesticide spraying. }\end{array}$ \\
\hline EDUCATION & $\begin{array}{l}\text { General bee knowledge } \\
\text { Spreading \& communication of } \\
\text { knowledge } \\
\text { How information is conveyed to } \\
\text { Wellington audiences } \\
\text { Online (Facebook \& websites), } \\
\text { community events (shows \& } \\
\text { guest talks) }\end{array}$ & $\begin{array}{l}\text { The education section discusses } \\
\text { how current forms of bee } \\
\text { information and education are } \\
\text { being distributed to Wellington } \\
\text { audiences. The design should } \\
\text { acknowledge the spread and } \\
\text { communication of knowledge } \\
\text { through online and community } \\
\text { event resources. Different } \\
\text { information topics need to be } \\
\text { portrayed and the identification } \\
\text { of appropriate communication } \\
\text { pathways should fulfill public } \\
\text { education needs. }\end{array}$ \\
\hline ENVIRONMENT & $\begin{array}{l}\text { Weather conditions } \\
\text { (wind, rain, temperature, sunlight) } \\
\text { Sustainability } \\
\text { Self sufficiency } \\
\text { (growing own food) } \\
\text { Climate change }\end{array}$ & $\begin{array}{l}\text { The environment section } \\
\text { identifies that the design should } \\
\text { portray the local weather } \\
\text { conditions preferred by bees } \\
\text { (wind, rain, temperature, } \\
\text { sunlight), climate and habitat } \\
\text { conditions influencing the } \\
\text { placement of beehives, practises } \\
\text { in sustainability and self } \\
\text { sufficiency and the broader } \\
\text { global concept of climate } \\
\text { change. }\end{array}$ \\
\hline
\end{tabular}




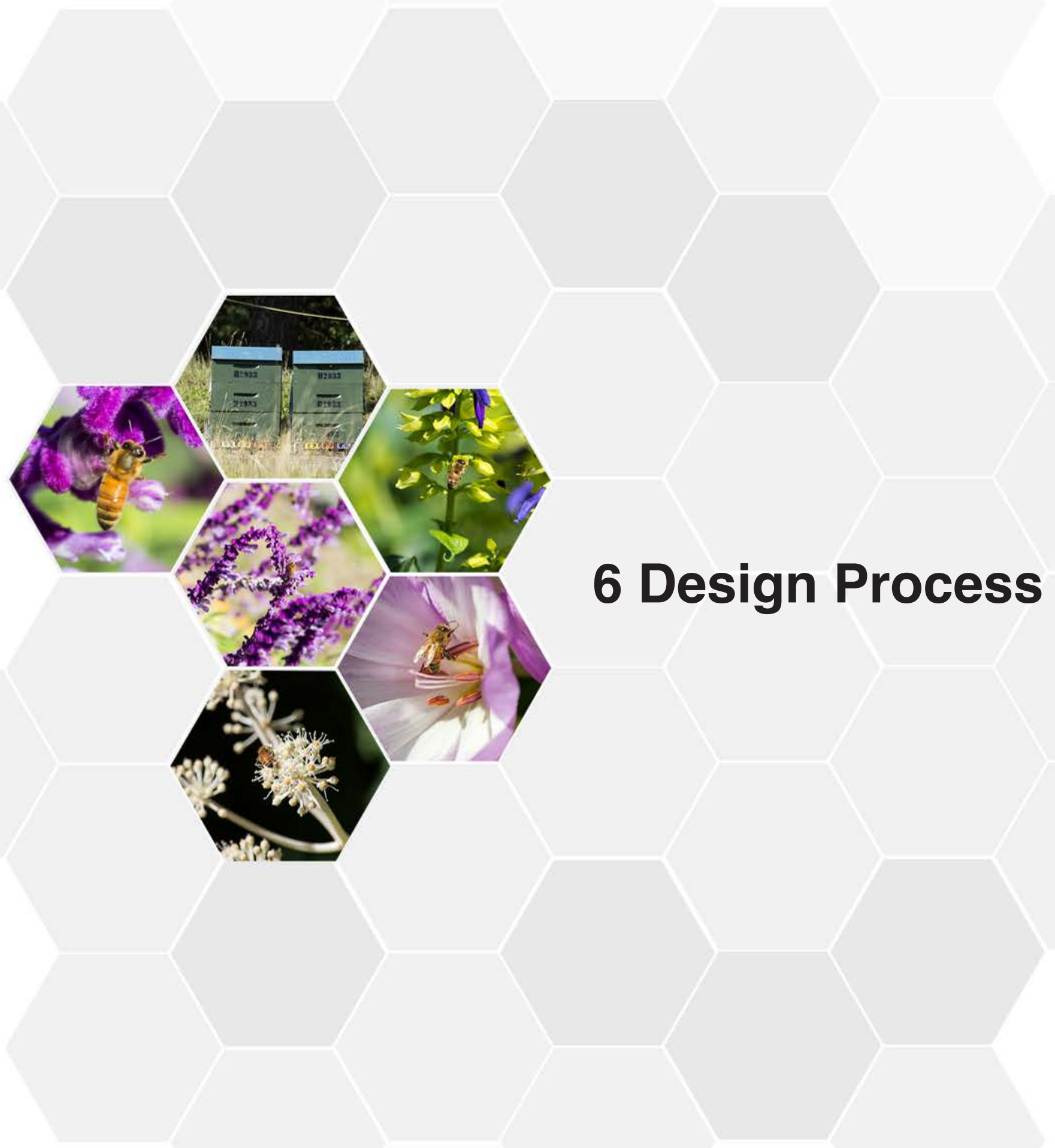




\title{
Communication Design Criteria
}

\author{
Jorge Frascara - Design Principles: Functional Requirements
}

\section{Perception \& Meaning}

The image must be strong enough to emerge clearly from its context. The images must be visually strong, that is, it should have a high internal cohesion, and should at the same time differ from the context surrounding it. Significance and relevance are major determinants for calling attention. Communication, in turn, does not end with attracting attention. Essentially, it involves making information understandable, usable, interesting, and, if possible, pleasing.

1. Clarity of form \& content (perception and understanding)

- The presentation of individual elements such as: letters, numbers, pictograms, diagrams, maps, charts, graphs, signs, symbols, signage, or control panels.

- The organization of communicational sequences, including categorization of complex information such as: timetables, study programs, learning aids, legal documents, rules and legislations, theater tickets, emergency instructions, instruction manuals, websites, or user interfaces.

2. Facilitation and stimulation of reading (publication design)

3. Consideration of cultural, social, economic, technological, and ecological aspects of all projects

\section{Language \& Signification}

The problem for the designer now is to avoid capricious selections and to develop a visual language that meets the needs of the project.

- Fitness to content (is there a good relationship between the topic and the visual presentation?)

- Fitness to context (are the visual language and structure appropriate for the people addressed and the situation in which the message will appear?)

- Quality of concept (is there an idea or only a layout?)

- Quality of form (is there a good perceptual organization?)

- Legibility/visibility (are letters \& images appropriately readable?)

- Craft (is the projects well presented?)

- Quality of medium (are the possibilities of the medium used well? Is it the appropriate medium for the project? Are technologies and materials well used?)

- Quality of the research (is the information collected sufficient, interesting, and accurate?)

- Power of the persuasive arguments (what is the perceived advantage for the public to remember the information provided or to adopt a new behaviour?)

\section{Aesthetics}

Fundamentally, before evaluating the aesthetic quality of a message, one has to ascertain whether or not the approach adopted is coherent to the public and the message in question.

Extending the Perceptual Time \& Memorization

In the usual environments in which visual chaos and ugliness tend to dominate, visual pleasure has an important role in the retention of the viewer's attention. Attract, retain \& communicate. Discussed conditions, a message that attract attention, communicates without contradictions, and retains attention on the basis of pleasure through a sustained time.

\section{Active Life of a Design}

A design with a high aesthetic value will have more chance of being kept active in the mind of its public. The quality of the environment is one of the social responsibilities of the designer. Our urban life is surrounded by design: architectural, industrial, and graphic. Communication design quality should be considered not only in connection with the specific scope of the project in question but also with regard to the cultural impact that it has on our daily life.

Contemporary, traditional, popular visual styles

Major concern in design because:

- It creates attraction or rejection "at first sight"

- It communicates

- It affects the perceptual time an onlooker dedicates to a design

- It affects the memorization of the message

- It affects the active life of the design

- It affects, in a broad sense, the quality of the environment 


\section{Prototype Design Criteria}

Evaluating Website Communication Design

\section{Target Tool for Audience}

- Applications / Online Platforms

- Quality of medium (are the possibilities of the medium used well? Is it the appropriate medium for the project? Are technologies and materials well used?)

- Fitness to content (is there a good relationship between the topic and the visual presentation?)

- Fitness to context (are the visual language and structure appropriate for the people addressed and the situation in which the message will appear?)

- Quality of concept (is there an idea or only a layout?)

\section{Design Media/Technology}

- Website Interaction - Communication Design

- Perception \& Meaning

- Clarity of form \& content (perception and understanding)

- Facilitation and stimulation of reading (publication design)

- Consideration of cultural, social, technological, and ecological aspects of all projects

Social Media - Usability

- How easy is it to use the device? How easy is it to get here? How easy is it for me to know where

I am and how to get back to where I came from?

\section{Language \& Signification}

- Quality of the research (is the information collected sufficient, interesting, and accurate?)

- Power of the persuasive arguments (what is the perceived advantage for the public to remember the information provided or to adopt a new behaviour?)

\section{Graphic/Visual Design}

- Craft (is the projects well presented?)

- Legibility/visibility (are letters \& images appropriately readable?)

- Who is the audience (will audiences be willing to engage and respond?)

- How do people look at this? (to interpret an image, a form, or an object is to respond to it)

- How are these things embedded in a wider cultural context? (behaviours of the community)

\section{Overall Design Function \& Output Considerations}

- Key Values (what is the purpose of the message? In which contexts will the message be issued?)

- User Profiles (what are the implied messages? What are the media to be used?)

- Brand/Concept Message (consistent through design, images and content) 


\section{Prototype Purpose}

\section{Design}

The design prototype aims to communicate the scale and purpose of human actions necessary in a bee's life cycle, and their contributions to New Zealand's nature and culture. This visualises how the bee can symbolise a crossing of purpose and positive intent between humans and ecological systems. The purpose of physical and online interactions aims to normalise the purpose and resources bees aid in and to understand their contributions and how we are helping/hindering these. Whilst showing layers and perspectives of the bee community and encouraging human interactions to forge the path for future successes - from bee survival/ growth to the production of honey and education throughout these stages. There is a main focus on Wellington populations and the importance of bringing local communities together through ideas of sharing resources, planting sustainable crops, and education through which bees can help us communicate. The target market includes Wellington's student and young professional residents, as the communication tools includes online and social media platforms, popular

\section{Photography}

The major influence of photography in my thesis visualises different scales aimed at creating insight into a honey bees daily routine and how they experience the world. The difference is scale shows a human perspective versus that of a bee, especially in public spaces around central Wellington. Normally bees are too small to notice among a large grouping offlowers, so these images target natural everyday interactions. The aim is to encourage and potentially heighten interests in human observations in these areas, making the public more aware of their surroundings and the importance of bees in these areas. The enhanced scale photography of a single bee exaggerates their beauty and magnificence on a larger scale where the human eye is able to
The individual bee represents up to 50,000 other workers in the same beehive and can travel up to $5 \mathrm{~km}$ away from hive. The realisation of a single bee being a small part of thousands, shows how important this scale is to understand and that every bee only contributes a small amount to the overall beehive function. These massive systems of bees are locally unrecognised as they are often hidden or located on private residential and commercial land. Access to the Wellington Botanic Garden hives has increased the likelihood for the public to interact with bees and to learn about their processes through signage and observations.

It is really difficult to take images whilst surrounded by large numbers of bees, as the correct beekeeping suit must be available and worn to ensure personal safety as well as the safety of the bees. Due to this barrier, I decided to focus on ordinary experiences in public spaces, where I could access sites on a regular basis and contextualise the images within a familiar Wellington location. The images of public beehives signify the importance of beekeepers and their role in maintaining the wellbeing of our bees in local ecosystems and understanding the influence they have on our environments. The positioning of these hives is important in the pollination of surrounding native flora and consequently New Zealand's biodiversity and sustainability of our land. The media of photography visually interprets the current presence of bee populations in Wellington, and the large scales illustrate the perspective of the bee contrasting with the way we view the world. The curation of these images aims to tell a visual story about our current ecological state in order to encourage a public curiosity. Capturing a different perspective of another person taking a photo addresses the social media and technological component of the output, and positions the user in the physical beehive location. This shows how the target audiences would interact with the bees, including taking images and sharing their experiences with others online. 
Figure 8. Human Perspective of Bee at the Wellington Botanic Gardens, by Author
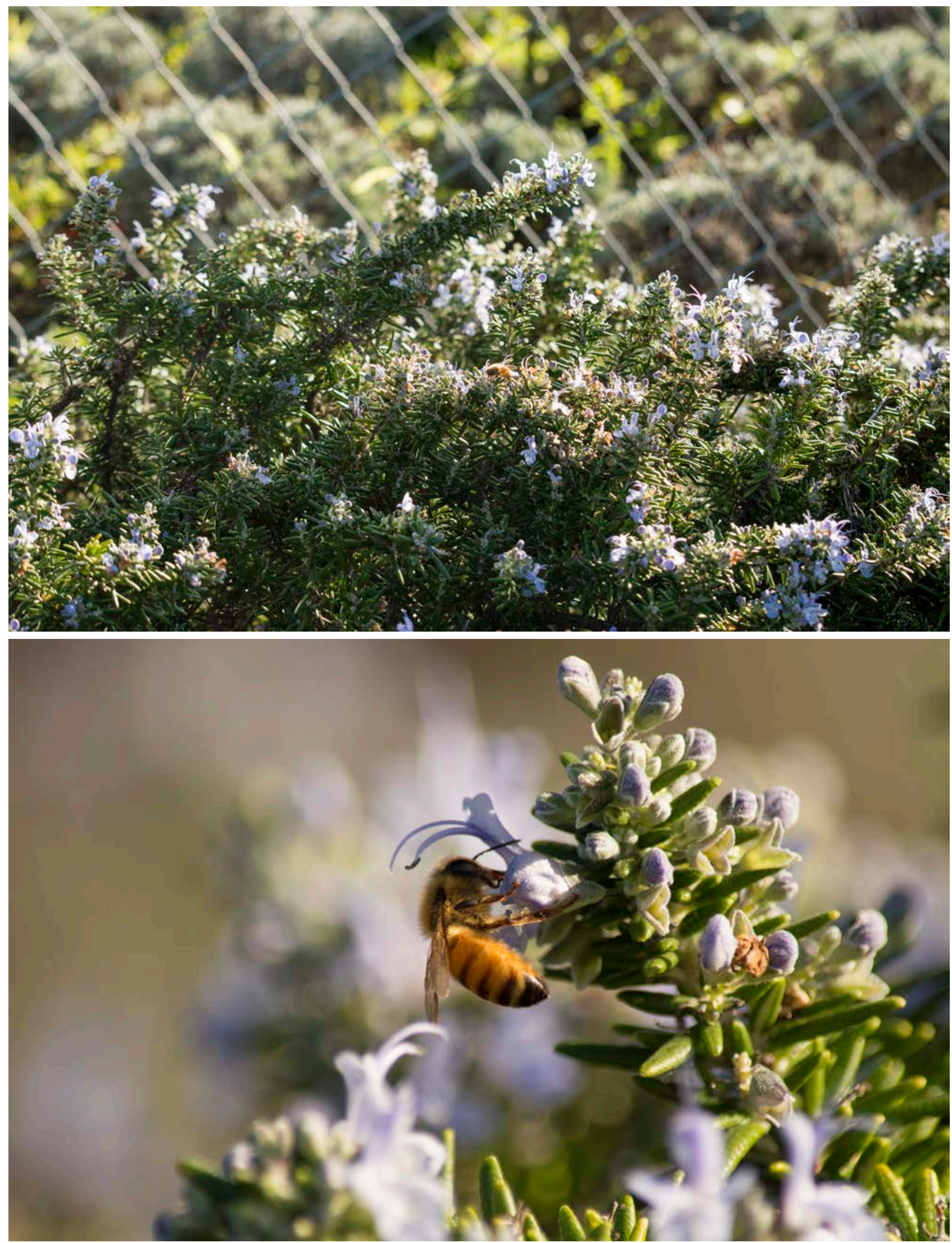

Figure 9. Bee Perspective at the Wellington Botanic Gardens, by Author. 


\section{Design Iterations}

The purpose of these iterations is to design represents under the key recognised themes. wireframes that bring structure to gathered The feedback from stakeholders addresses information and can also be used in interviews to which content they found beneficial or not receive feedback from key stakeholders. As each striking enough, storyboarding each key idea iteration progresses, an idea of similar topics and to refine and simplify the output visuals. I really content is pulled together, each evolving from wanted the visuals to be sequential in telling a the analysis of stakeholder views through to the visual story of current honey bee concerns, as visual interpretation of these ideas. This enables it represents the journey through the information the viewer to visualise the actual content on a but also poetically interpretes the life and mockup website, with design recommendations understanding of a bee. Each separate visual focused on the content and images rather than aims to evoke a different response, yet form an a working system. The layout is important to overall understanding of ideas to aid in raising structure as it demonstrates the hierarchy of an awareness focused on these imperative information and visualises ideas that each page aspects of our bee populations.

BEGINNING

1. HOME PAGE - THE HIVE

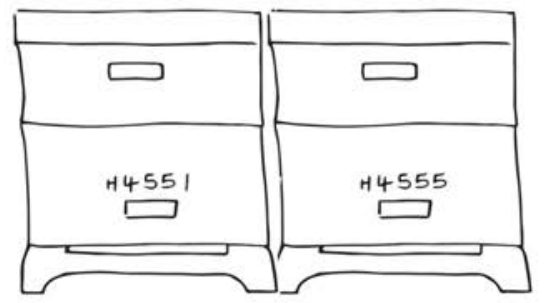

3. LOCATIONS - EXPLORING

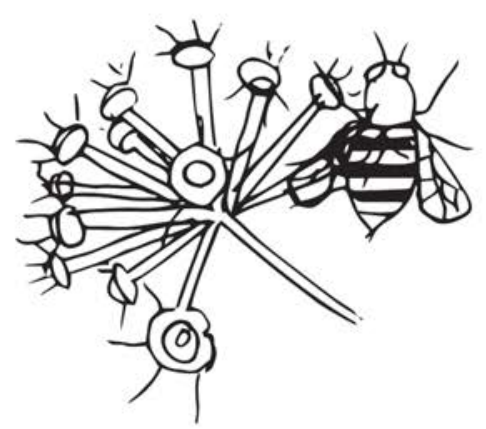

2. ABOUT - BEE TYPES
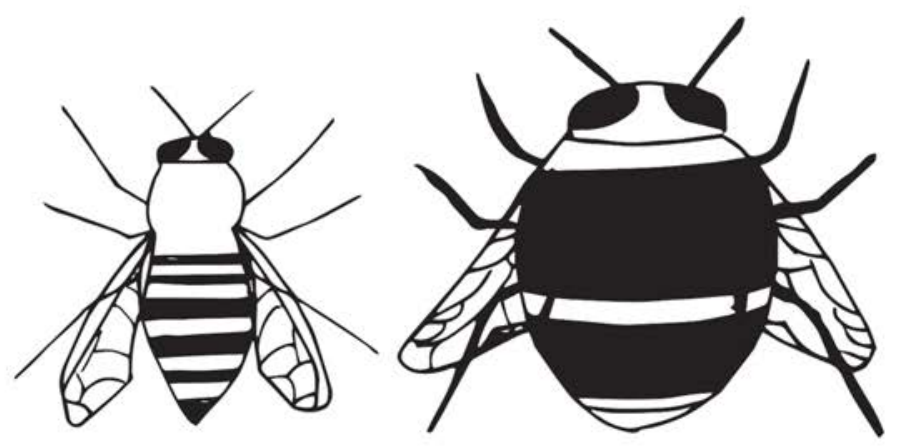

END

4. YOUR BEES - EXPLORE > HIVE

5. PRODUCTS 
DESICN ITERATION NC I

WHAT'S THE BUZZ

- public website

collation of experiences

\section{HIVES}

(1) cocation mappinc. NELLINCTON CENTRAL

QR CONE
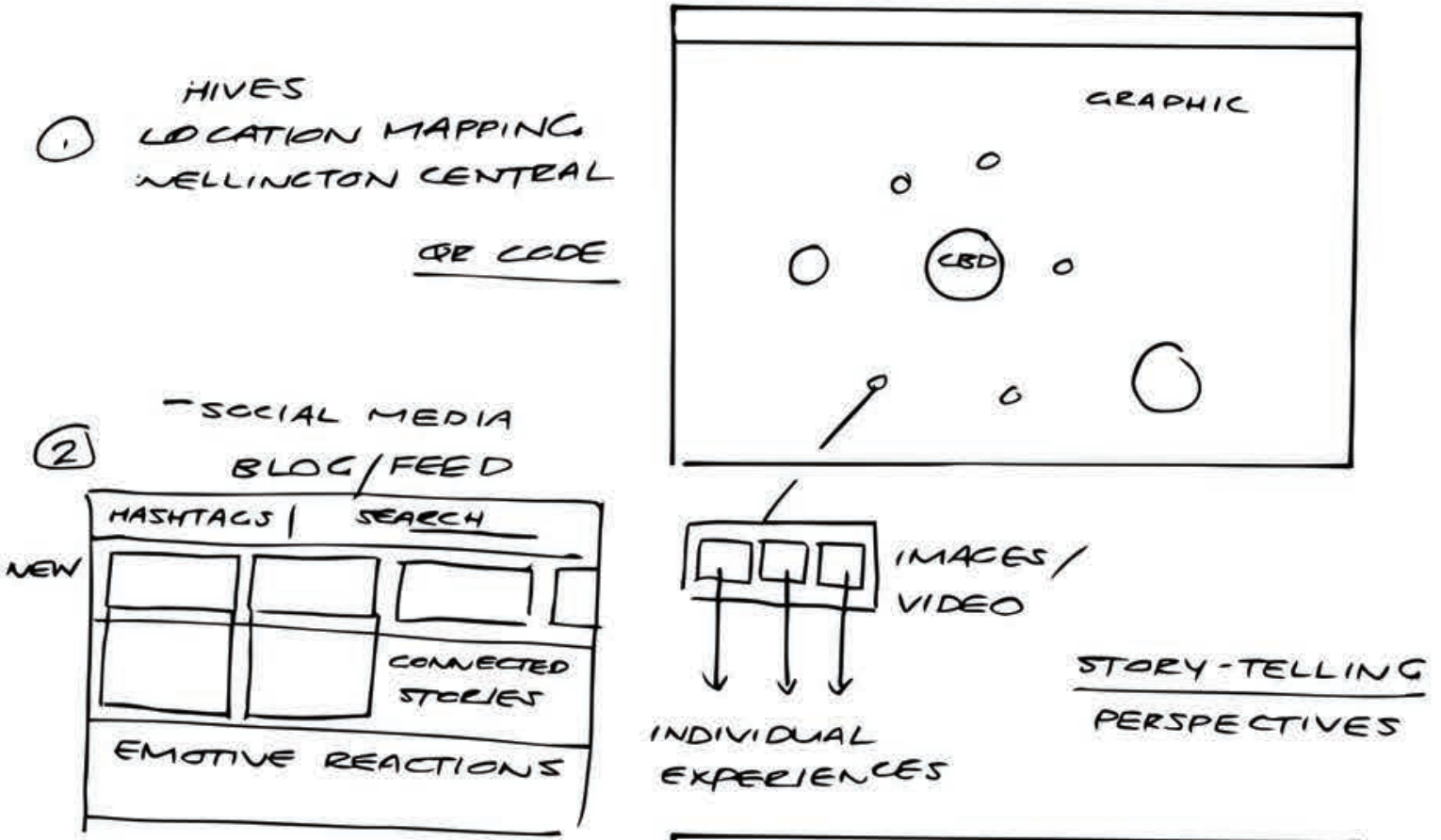

INDIVIOUAL

EXPERIENCES

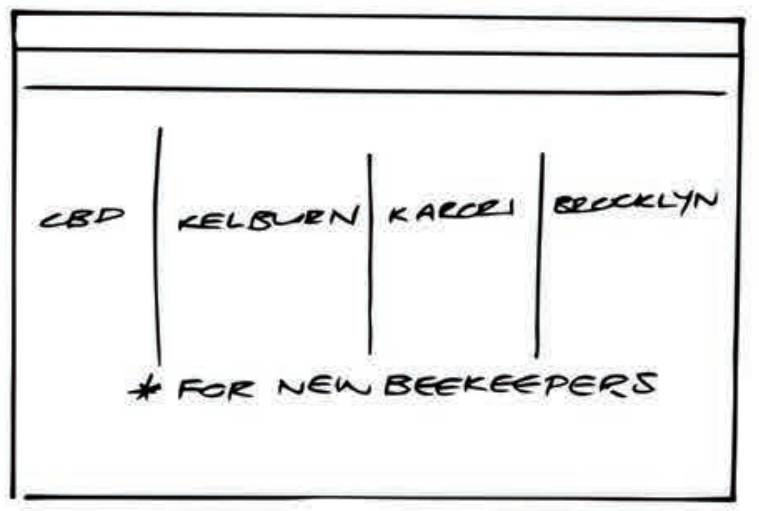

(3)

LCATIONS

SOET BY HIVE

- stre INUTAATION/

PROMOTIONS EVENTS

NESSTTE

- CONTENT FRAMEUCEK

TESTING -QUESTIONS

DESICN CKITECIA

KNEULEDC OF BEES

AWARENESS

6 Points

SUSTAINABILTI

CONMUNTTY

- Terget Toelfer

GRBAN NLG.

ENCACEMENT

Andience

- Hou to addrese

- Desian -technacey

them in website

social Media/communication Desigh

- Graphil desian tool

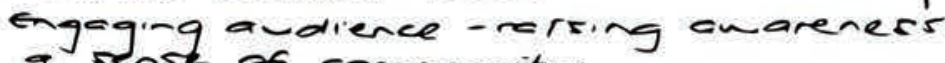




\section{ITERATION 1}
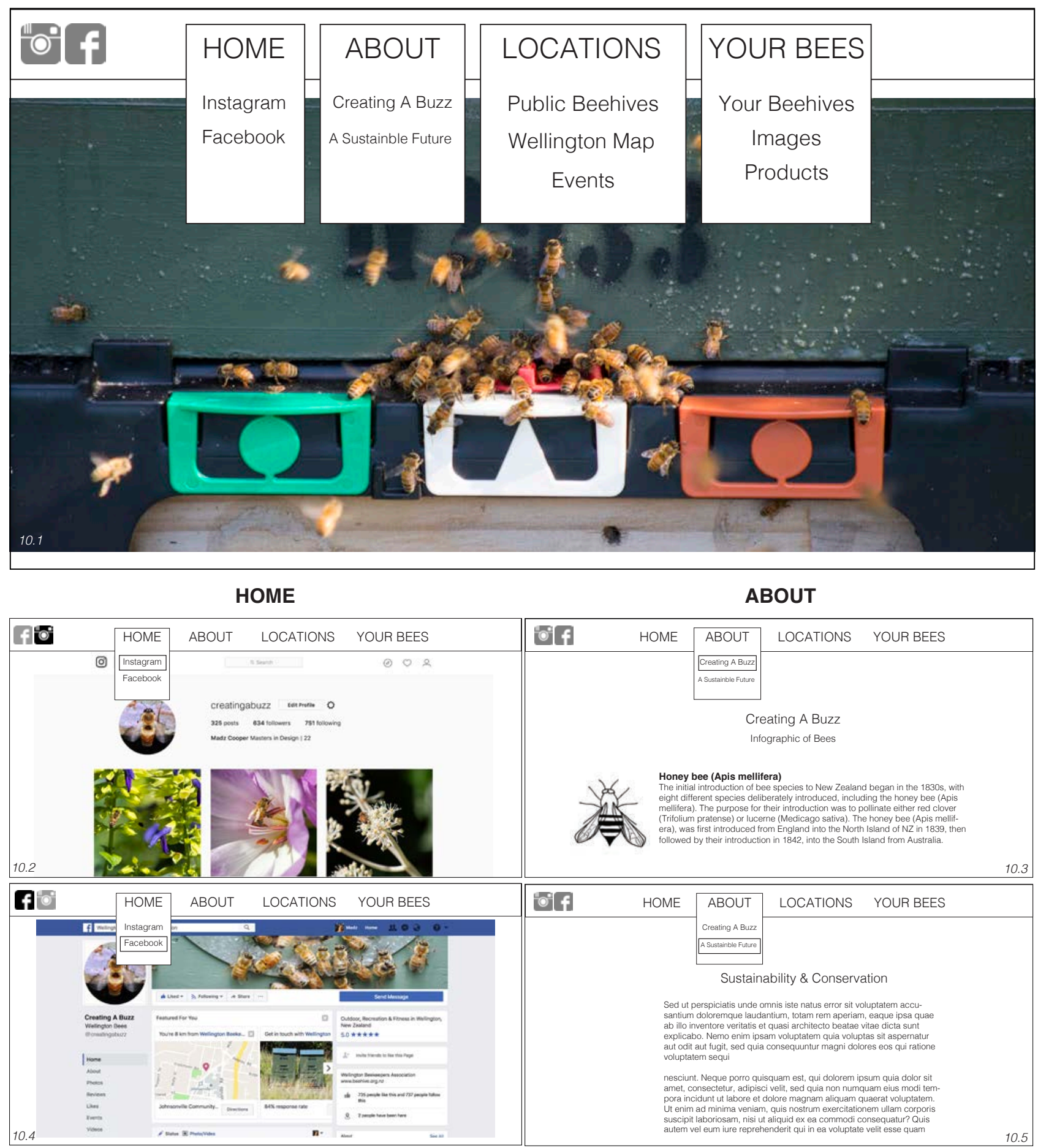

Figures 10.1 \& 10.3 images by Author, Figures 10.2 \& 10.4 Screenshots from Instagram \& Facebook edited with images by Author 
LOCATIONS

YOUR BEES

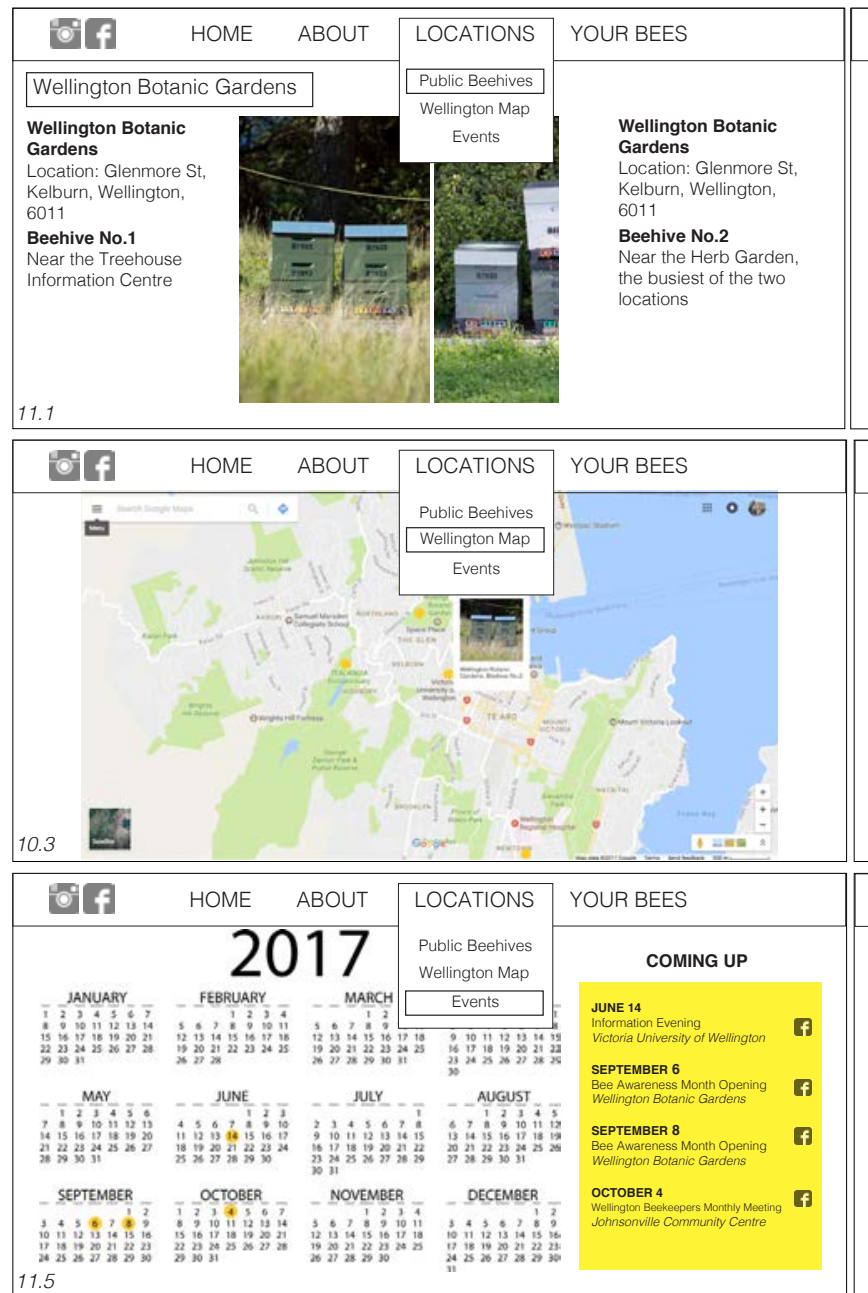
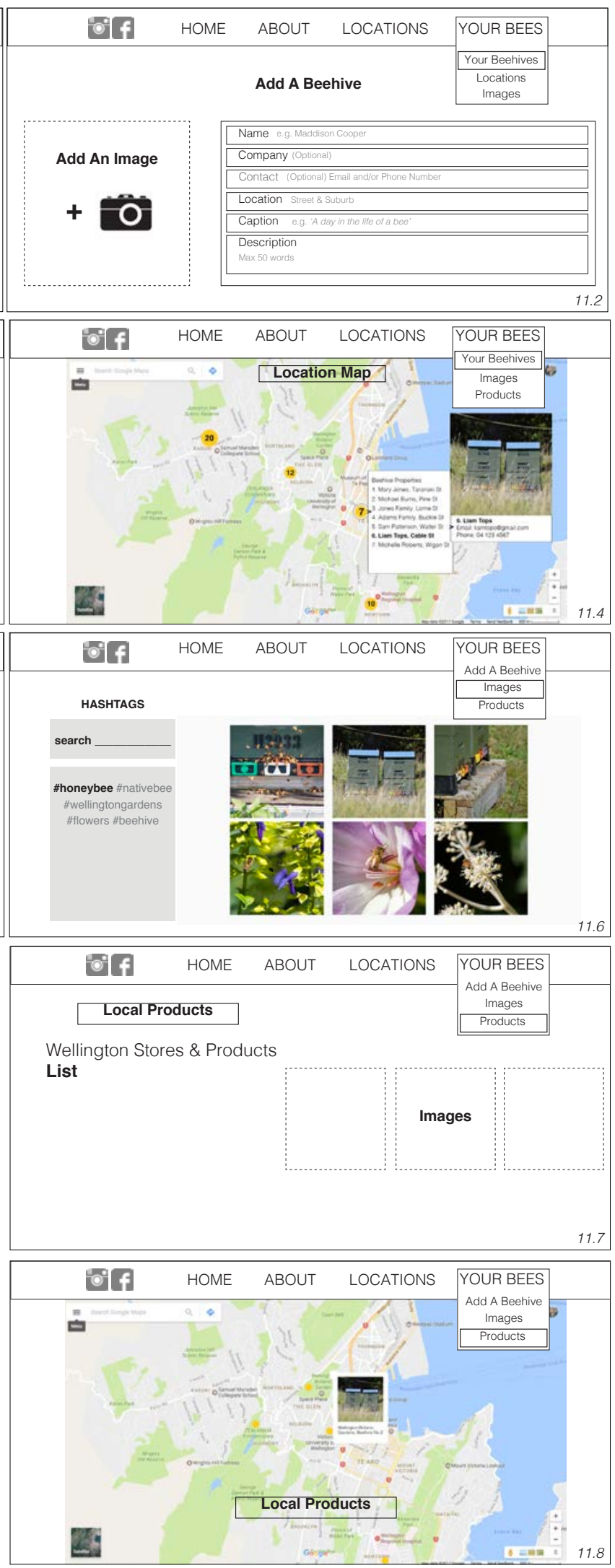

Figures 11.1 \& 11.6 images by Author, Figures 11.3, 11.4, 11.5 \& 11.8 Screenshots from Google edited with images by Author 


\section{Design Feedback \\ Wellington Beekeepers Association}

(Personal Communication)

You might want to consider the solitary bee, which is one of the things the Botanic Garden wanted me to do something about solitary bees. I said well you can't cultivate the solitary bees, you can provide the conditions for them to be encouraged - which is generally exposed clay banks that they can drill holes into, that will help but you can't actually cultivate them or doing anything more than just encouraging them or providing them with the right environment.

Design Suggestion: have photos of the different types of bees (bumble bee, honey bee and wasps)

Already exists: tagging photos and beehive locations and view other peoples hives

You can actually do that through the Wellington club website

Beekeepers normally try to hide their hives, because people are very easily intimidated by just the sight of hives. And that's often why they are painted to blend in, and they're painted or sited where they can't be easily seen, off the track. Whereas the Botanic Gardens didn't want that, they wanted them to be visible. The rope barriers and the slope we have got them on above the new childrens playground. So far it has worked out quite well, so far we haven't had any instances that I know of where people have been stung or interfered with the hives. It could happen as they become more prominent, they've been there 6 months and they started off as a nucleus just couple of boxes, but in time they will grow to twice that height and therefore the bee numbers will be twice as what they are now.

There is an observation hive at the new learning pavilion that is going to be installed at the Botanic Gardens, the whole idea is to educate the public, get them more involved. A display hive at the Wellington Zoo already exists (meet the locals) bees are seen as another domestic animal that has been introduced to NZ for the benefit of the country. Bee facts, mainly for the kids, teaching city kids - bees are seen as just another animal that have formed a key part to NZ's agricultural heritage. Emulate at the Botanic Gardens, have to find a way to manage that hive safely without people being involved. Maybe all the information that goes with it should be the part and parcel of the experience.

Design Suggestion: In my opinion you don't want to overburden them with too many facts, there are an awful amount of facts you could give them about bees, you can get those anywhere. But I think it's the bee itself, that action that is in my opinion fascinating. 


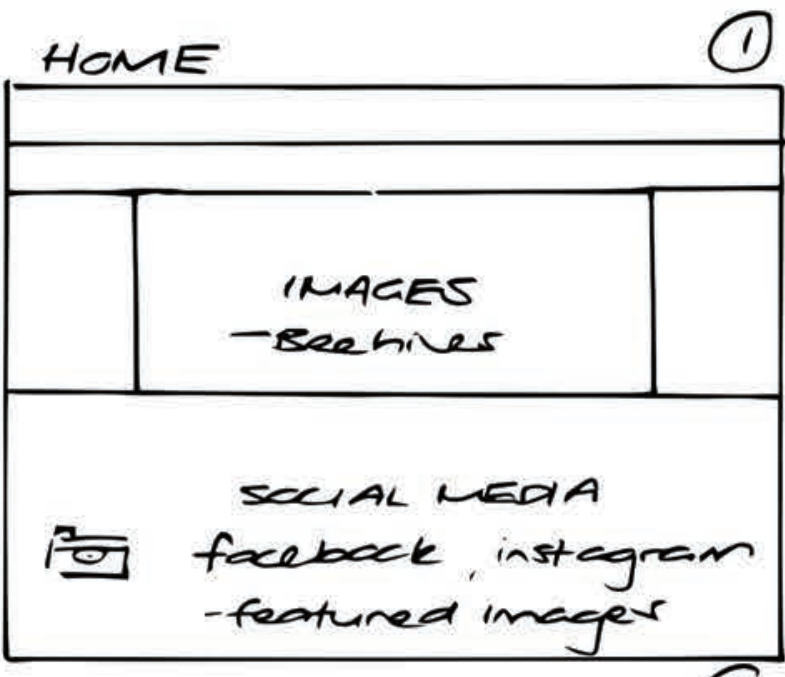

IMACES

ABOUT

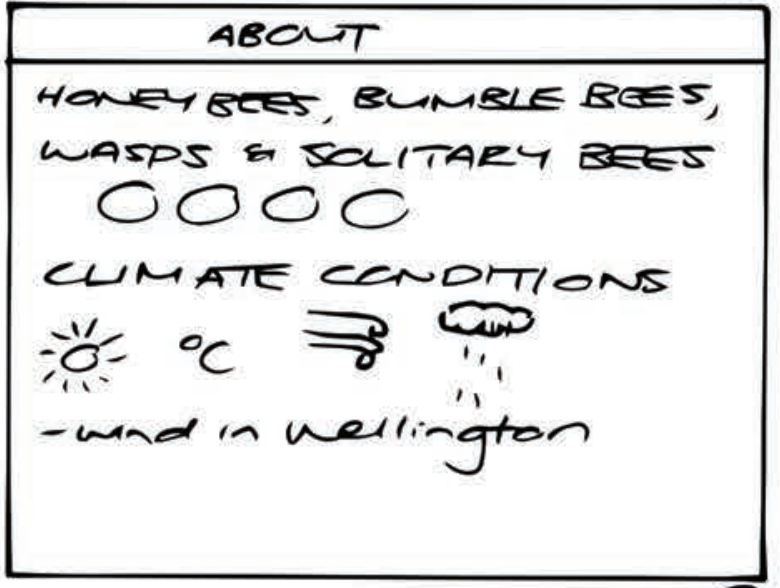

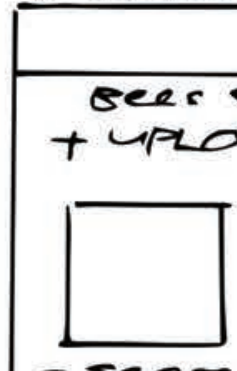

MACES

HOW WE CAN HELP

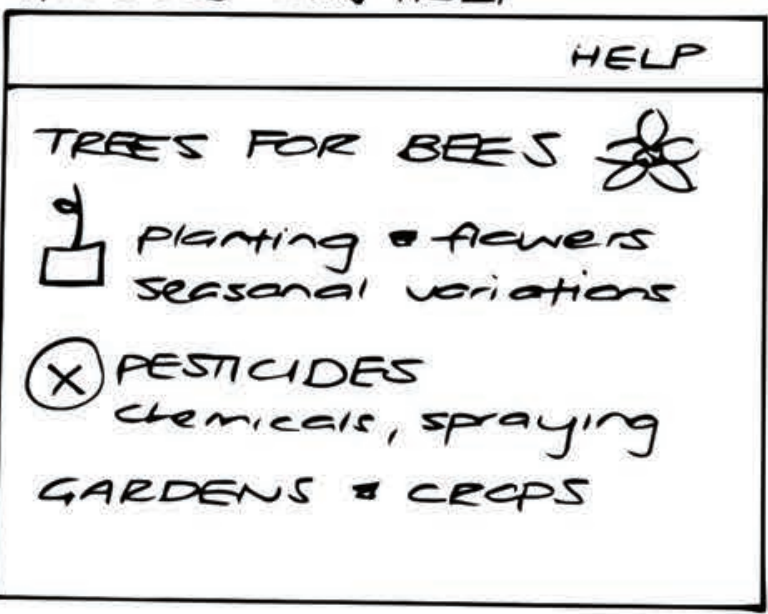

Instagrem ofecebck

we Feed.

- nelunaton map

iceations wral

$\downarrow$

EMTOVED -

Beenive

Lecotions aready exists

PRODUCTS?

LCCAL PRODUCE/

COMPANIES

STCCKISTS

$\rightleftharpoons$ raney

M PRCOUCTS
WELLINCTCN

SPECIFIC

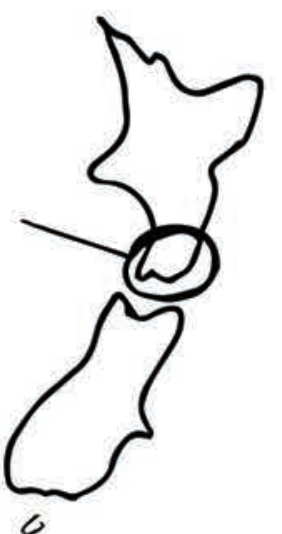


ITERATION 2
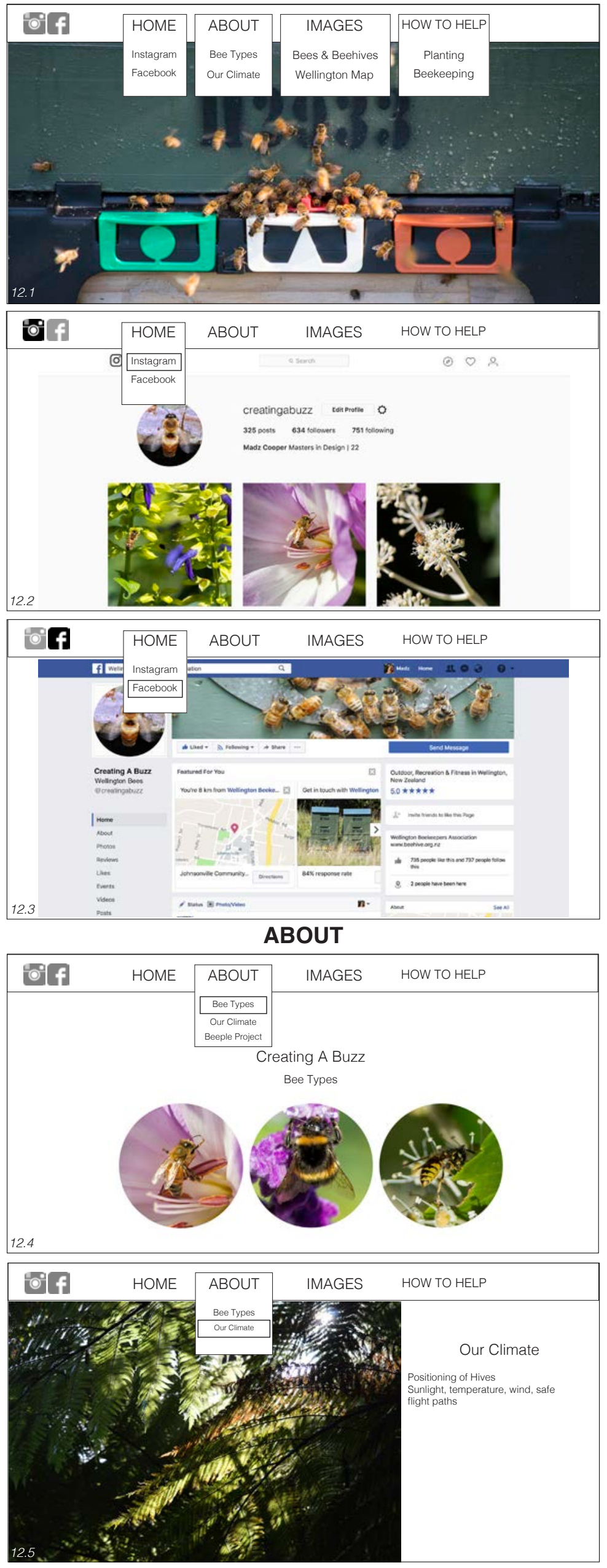

Figures 12.1, 12.4 \& 12.5 images by Author, Figures 12.2 \& 12.3 Screenshots from Facebook \& Instagram edited with images by 
HOW TO HELP

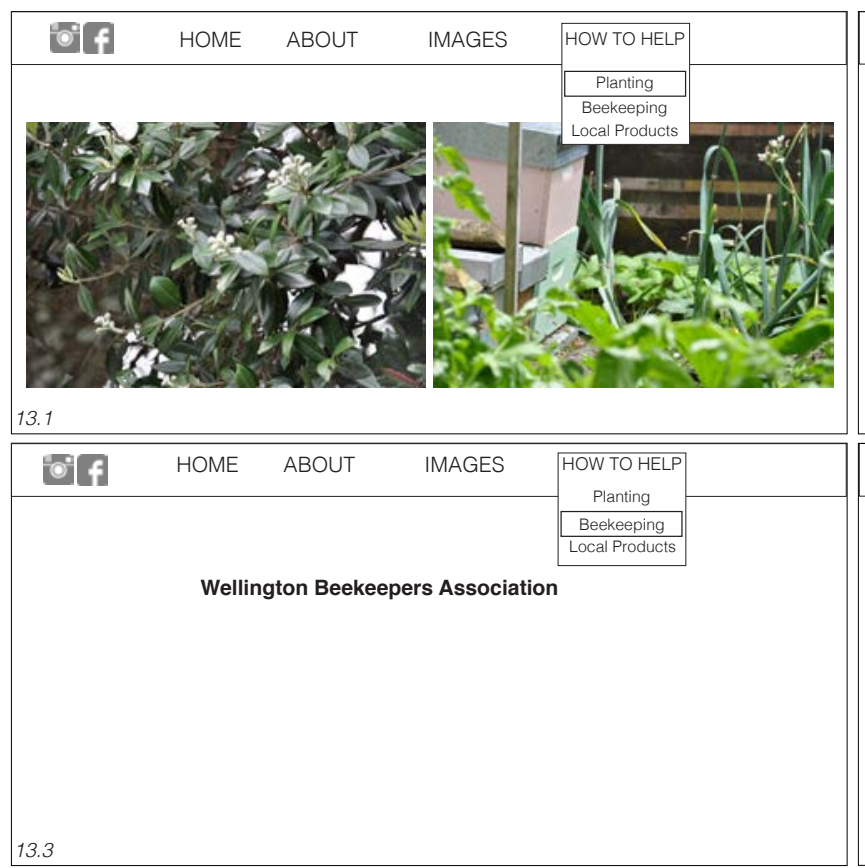

IMAGES
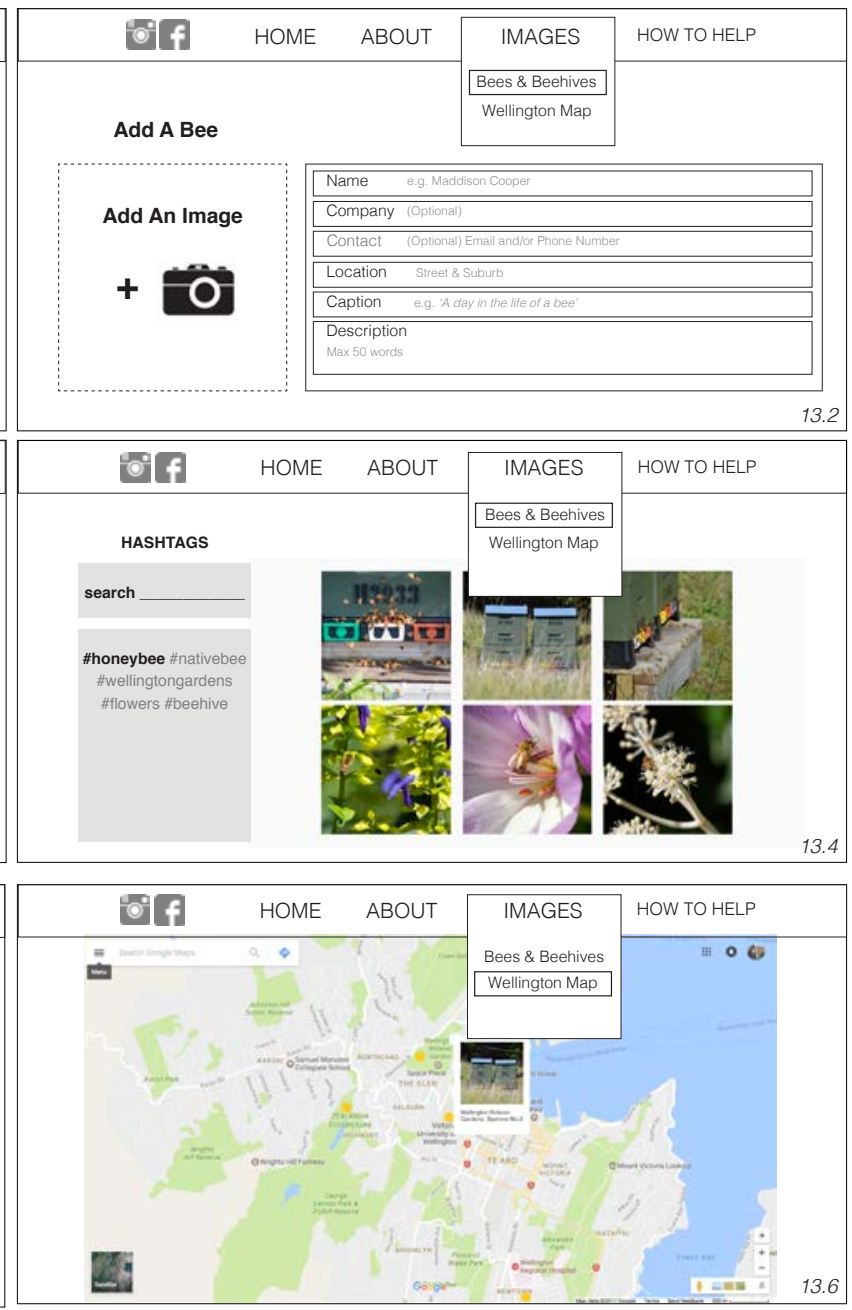

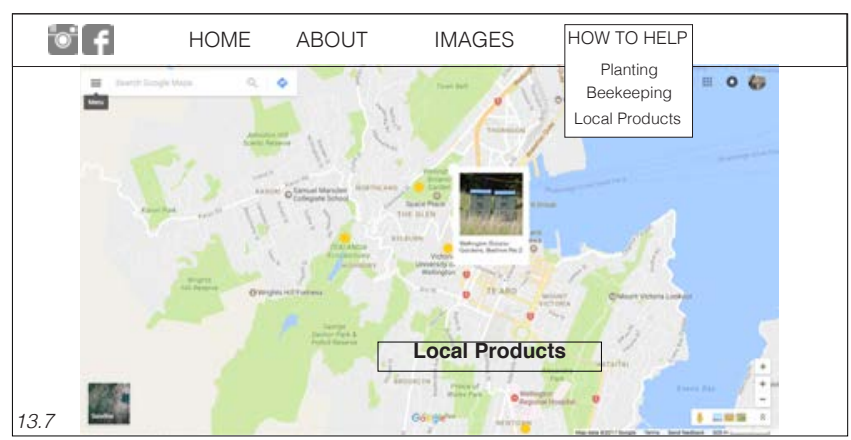

Figures 13.1 \& 13.4 images by Author, Figures 13.6 \& 13.7 Screenshots from Google edited with images by Author 
IMAGES

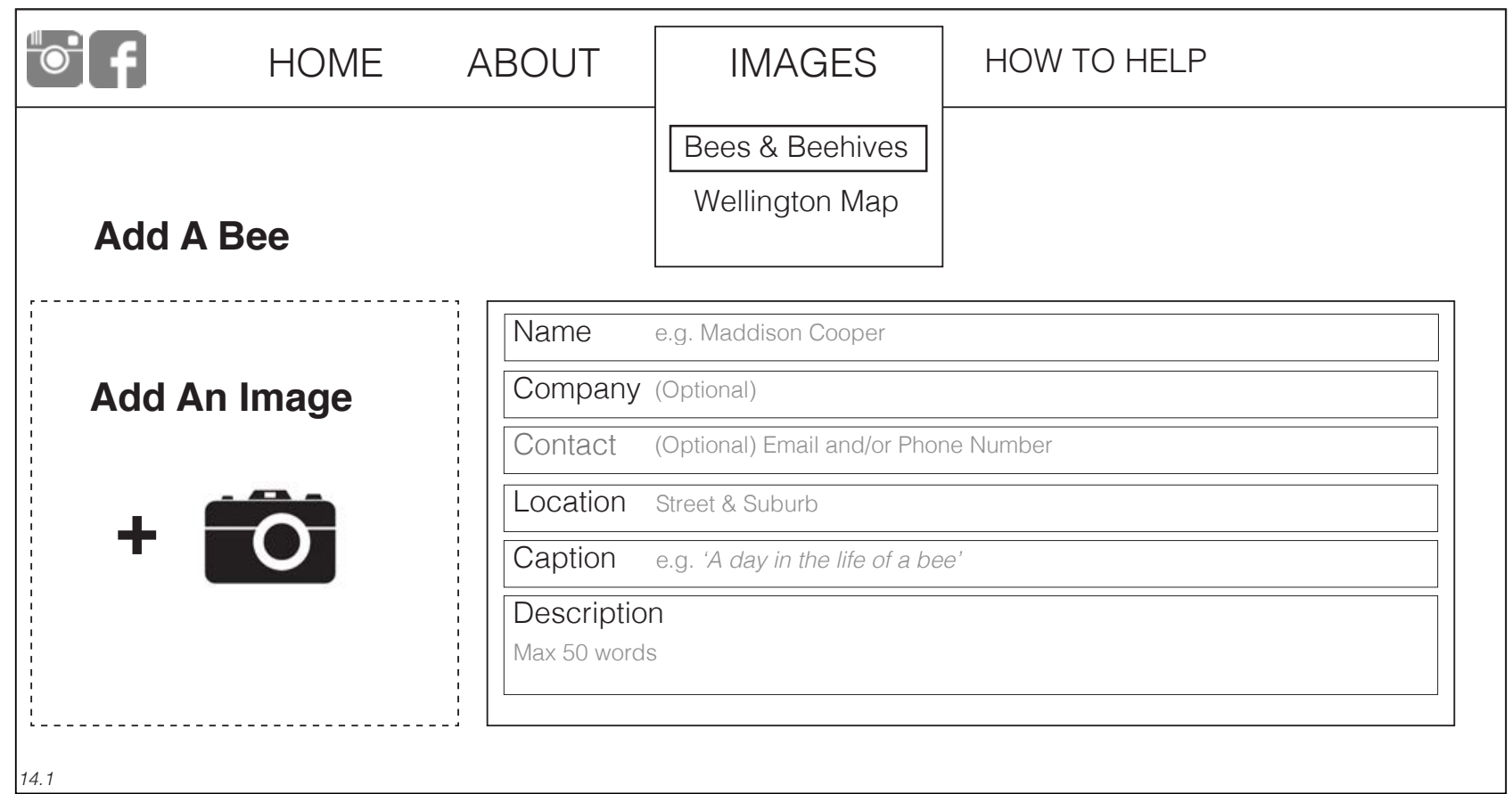

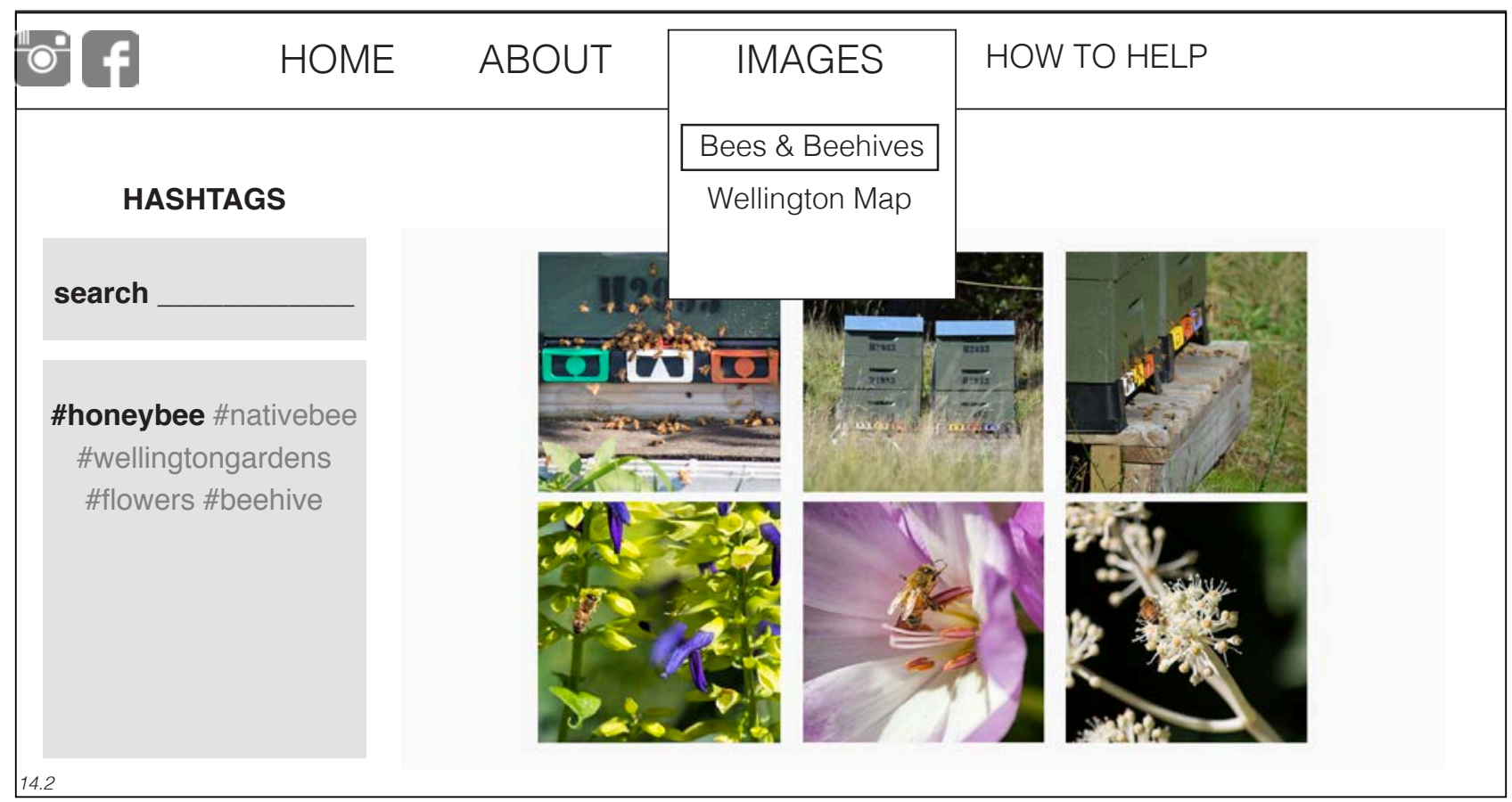

Figures 14.2 images by Author 


\section{Design Feedback \\ Beeple Honey Collective}

\begin{abstract}
A key suggestion from the first iteration feedback focused on the education and engagement of the public - "the whole idea is to educate the public, get them more involved." Reiterating the importance of bees and the application of public education, quotes from this discussion emphasised climate change and sustainable practises:
\end{abstract}

- "We need bees, we need populations of bees, we need ecosystems that are varied so that the bees can actually do a job, they need to move on as things flower. I think bees have a huge part to play in education around climate change, all of those things that are kind of getting really out of hand. We've lost touch, and the bees kind of keep us in touch. There is a sort of parallel between, and one of things that I get really excited about is looking at bee communities in their hives and reflecting that on society because we need to care for each other."

- "This is all part of that educational thing that we are actually dealing with an animal that needs certain conditions in order to be able to do it's job and we need it to be able to do it's job so that's all that takes and all that climate change stuff as well, it's a massive subject."

- "I think it is good that we are answerable at some point because I think that's just carelessness or lack of education around beekeeping is what's going to spread these diseases."

Other key suggestions related to the misconception and nature of human intervention: - "I think that bees are an amazing reflection of humans actually and community and how we should work together and work as a community." - "I mean like I say we are so dependent on them that is such a misconception to think that they are somehow dangerous to us - without them we are in danger, it needs to be flipped on its head I think."
- "There is something about bees that creates this sort of magic in bringing people together."

A particular concern was to address New Zealand's Manuka industry:

- "There is a lot going on in that mass bee business, side of things that are really just quite sickening actually - forgetting that it's an animal, that's the bit that bugs me we are exploiting these animals, forgetting that we are actually part of nature."

\section{Target Audience}

"I like to throw out crazy facts, especially with teenagers, like it takes a kilo of honey 4 million flowers, it's a phenomenal thing that they do. Half a teaspoon is a bee's life, and I love that it inspires people to look at bees a bit differently and start thinking about what they are actually doing, rather than just being something that's going to sting you."

\section{Design Suggestion Summary:}

Main Ideas:

- Community Involvement

- Personal Development

- Responsibility of human intervention \&

beekeeping skills

Bigger Issue:

- Climate change, overpopulation of bees and humans, urbanisation

- Mass farming of bees - unethical practices

- Unsustainable scale of Manuka honey farming and exploitation of bees - consumer and money driven

Goals:

- Sharing and helping among communities, self sustaining communities - healthy vegetables and seasonal variations in flowers

- Sustainability of the honey industry 


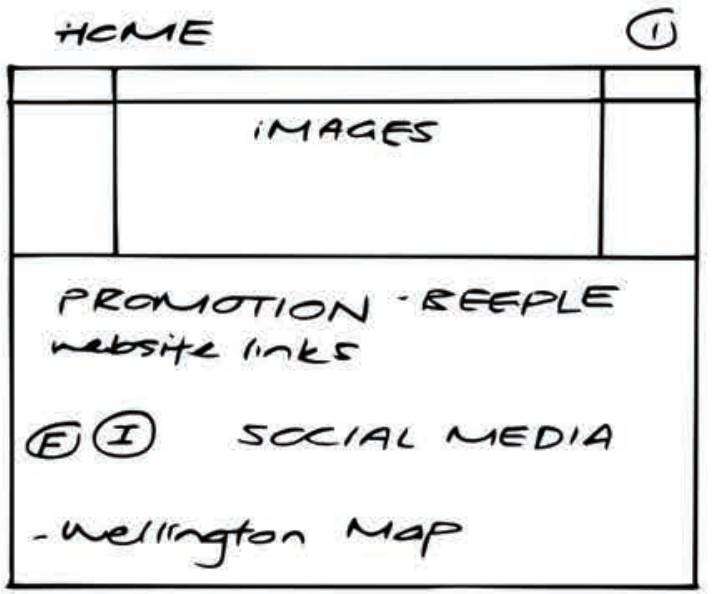
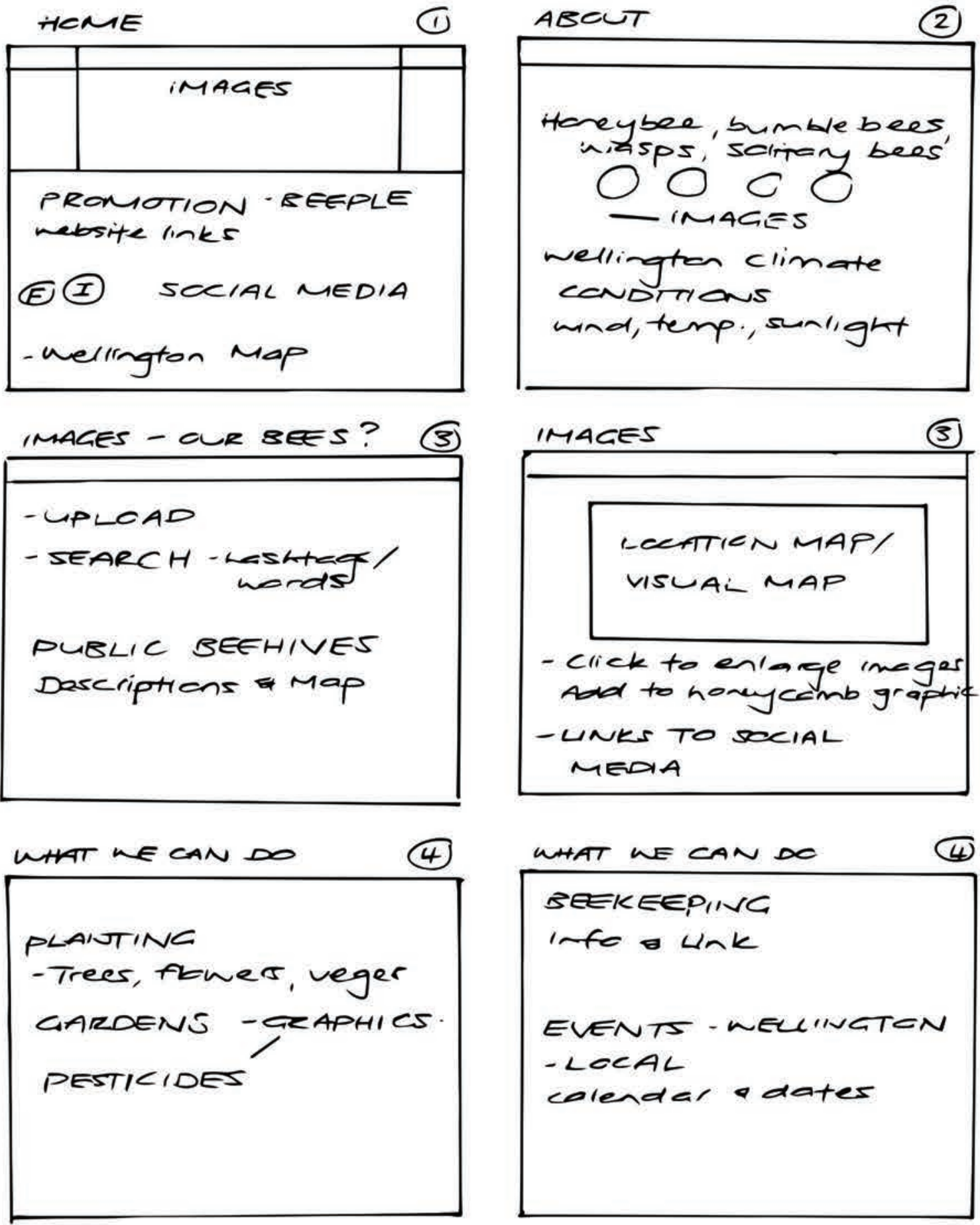

\section{ADDED}

ORCAIUISATIOMI FRCMOTION

1. BEEPLE

- IDEA OF COMinumTI/ STARINC

2. WELLINCTONS BEEKEEPERS

REMCVED

iocal PRCDUCTs 


\section{Iteration 3}

HOME PAGE
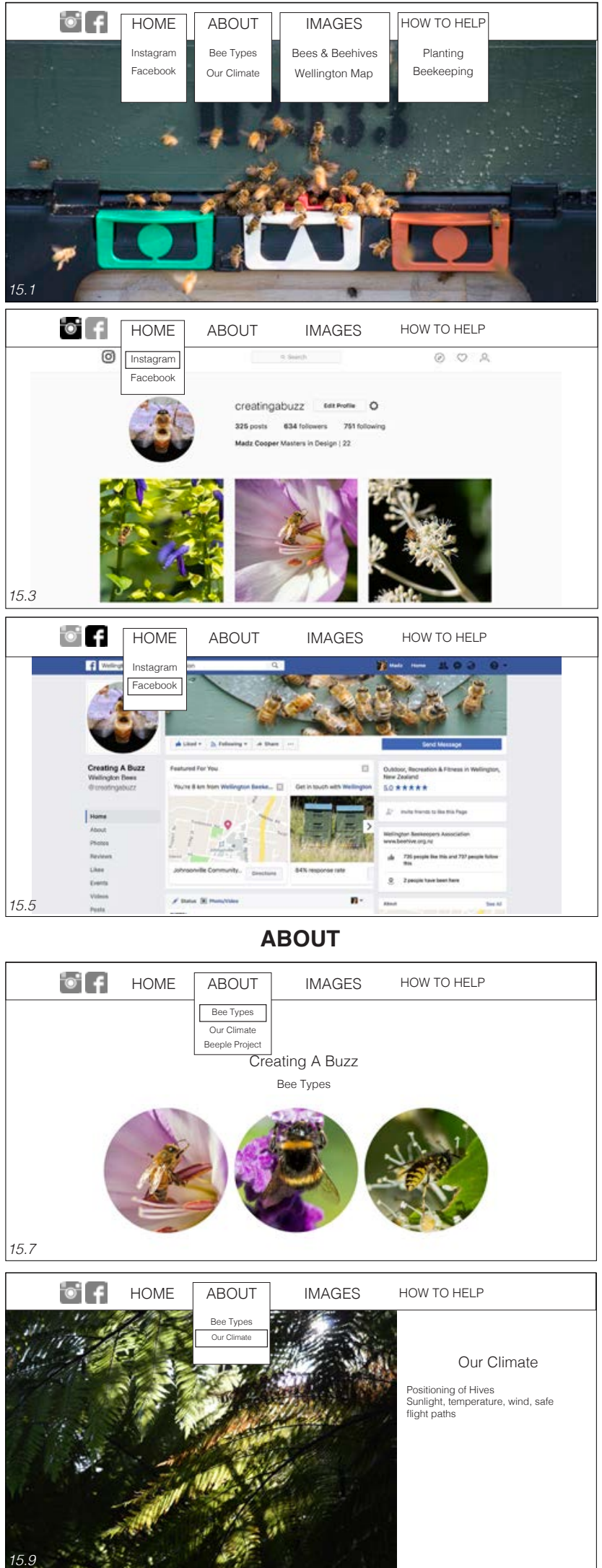

IMAGES
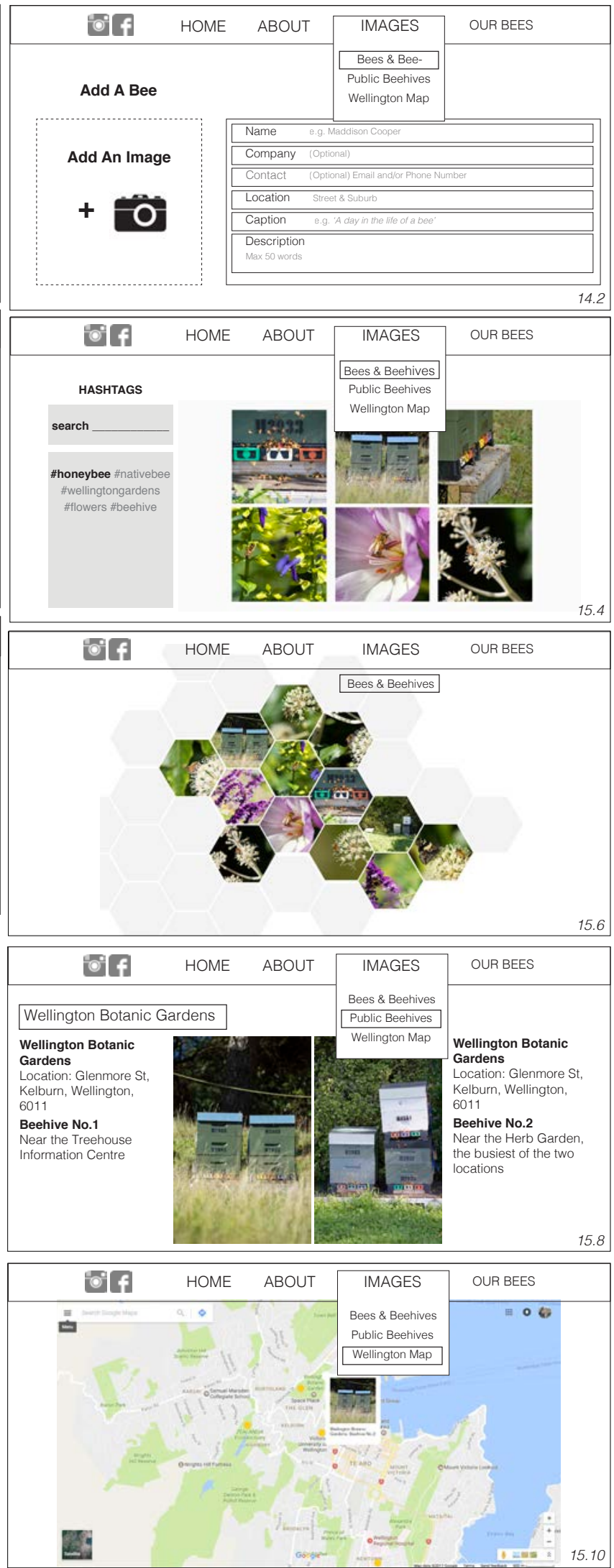

Figures 15.1, 15.4, 15.6, 15. 7, 15.8, 15.9 images by Author, Figures 15.3 \& 15.5 Screenshots from Facebook \& Instagram edited with images by Author, Figure 15.10 Screenshot from Google edited with images by Author 


\section{IMAGES}

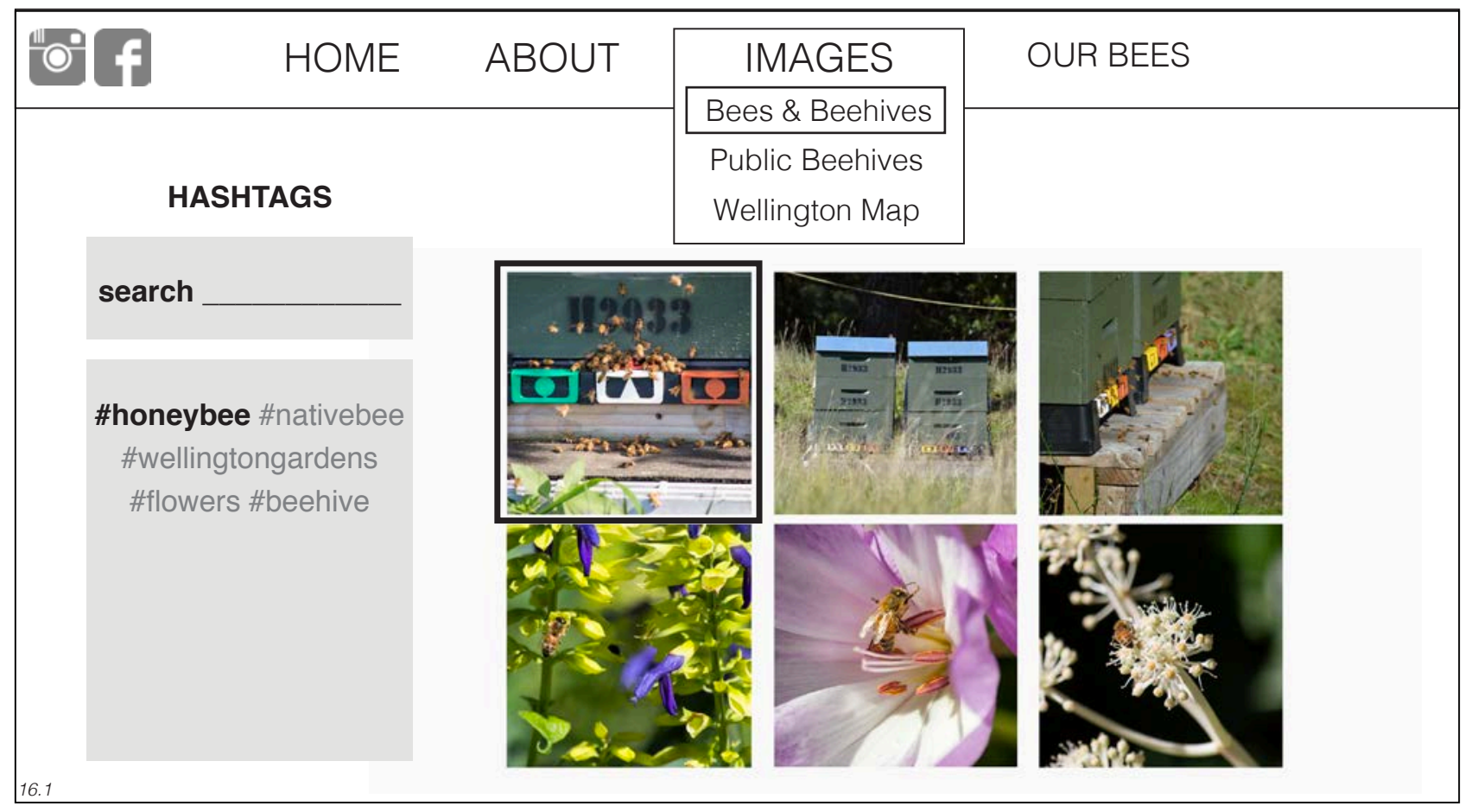

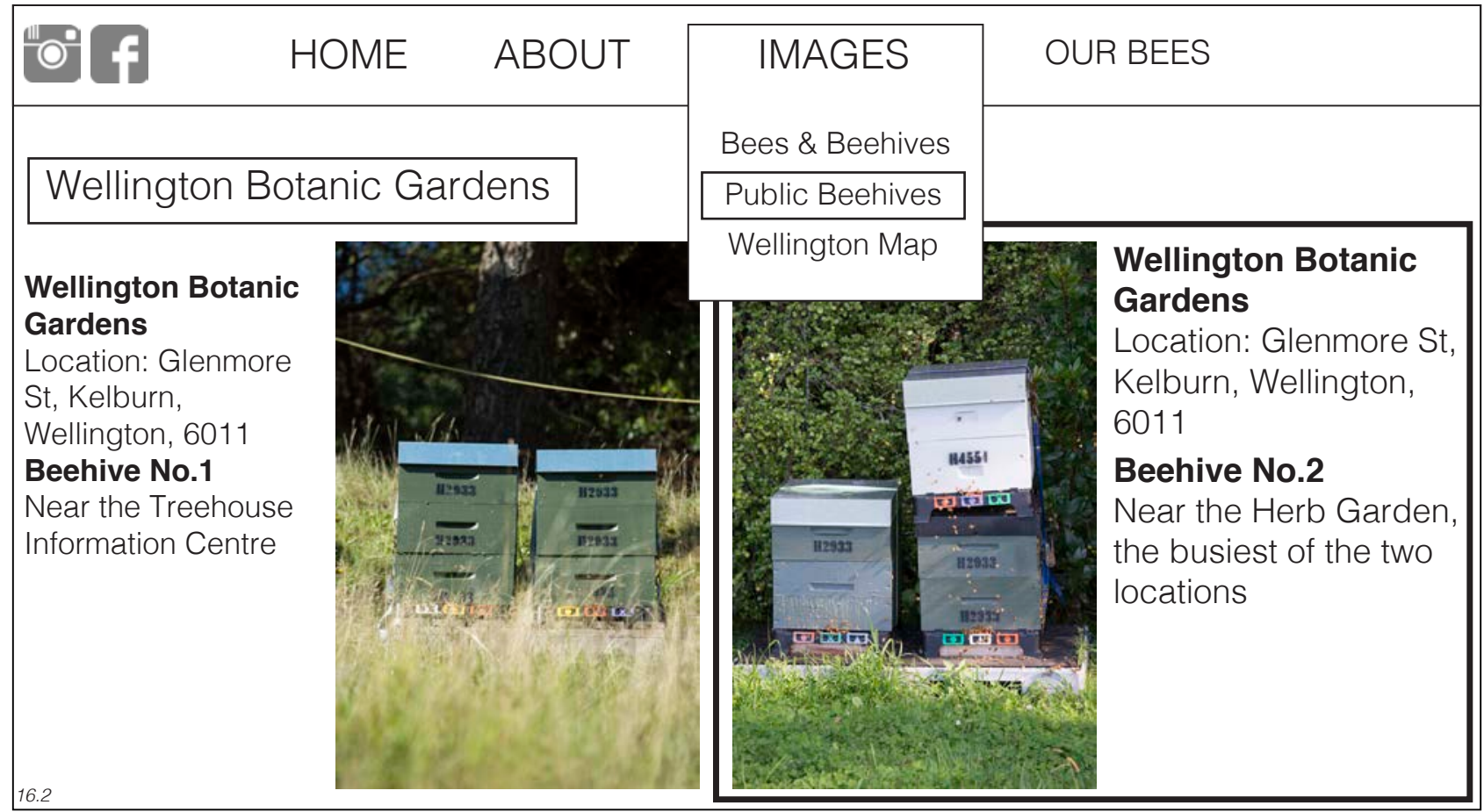

Figures $16.1 \& 16.2$ images by Author 


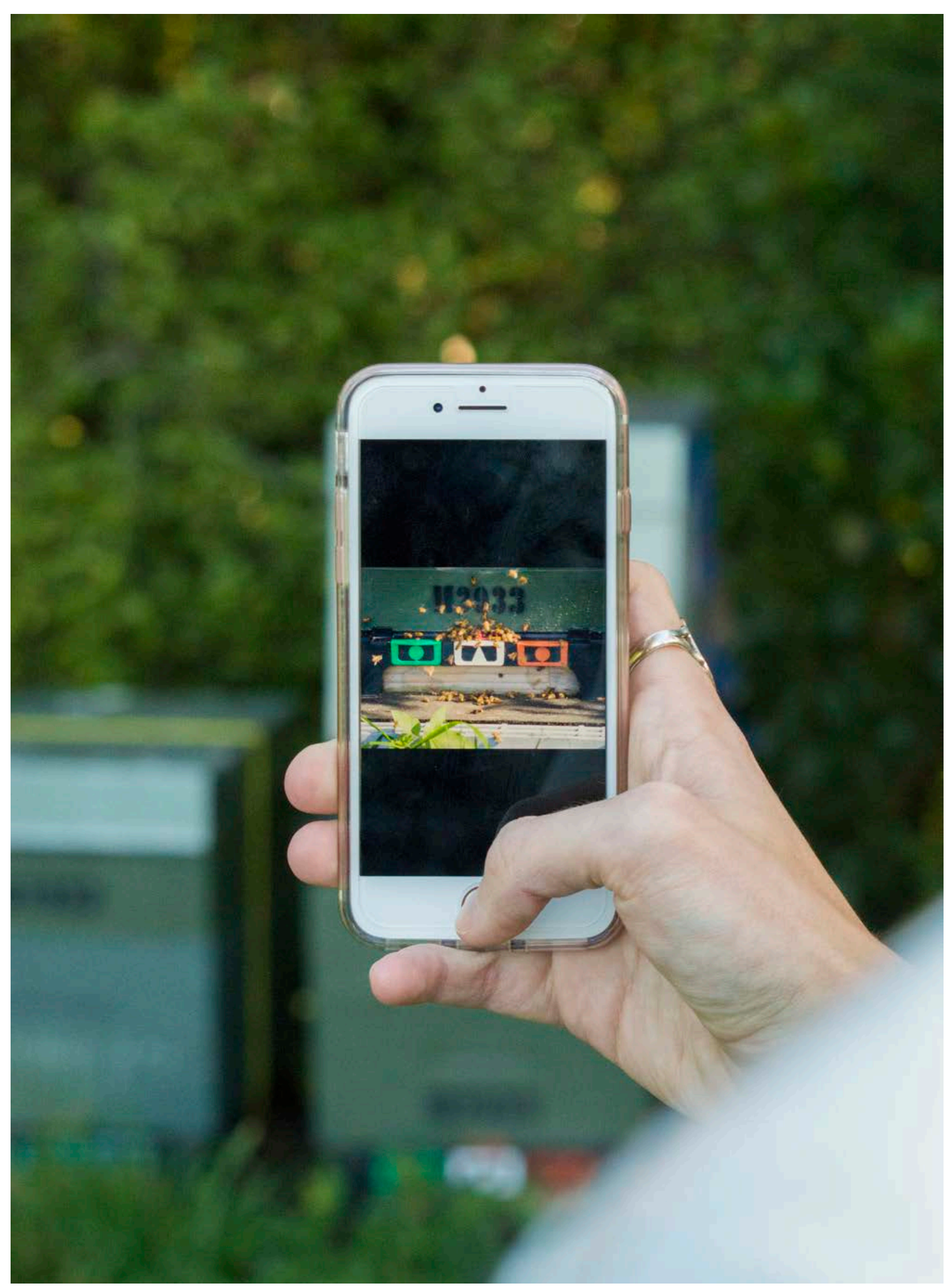

Figure 17. Public Interaction - Phone Photography at Beehive Locations, by Author 


\section{OUR BEES}
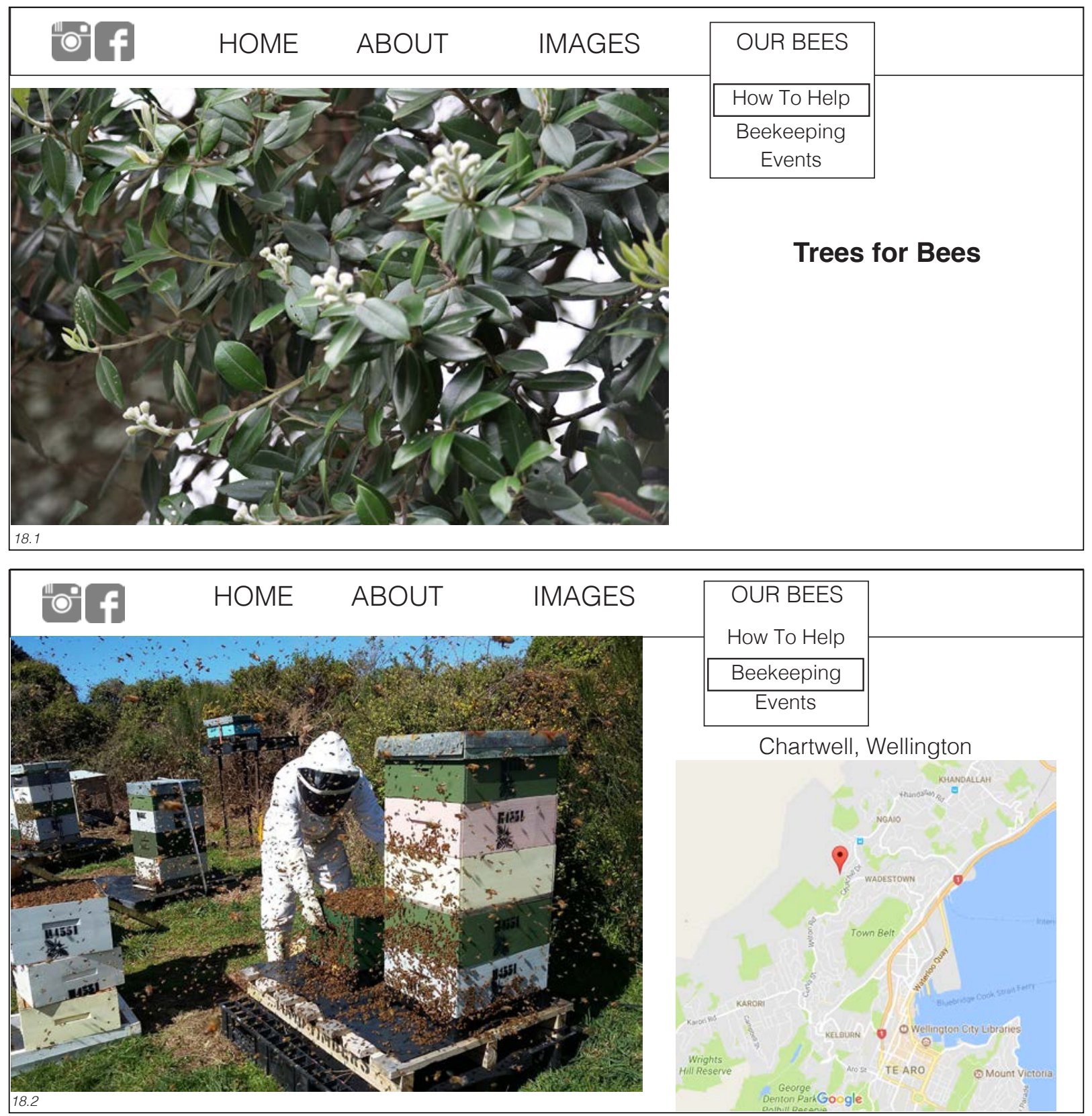

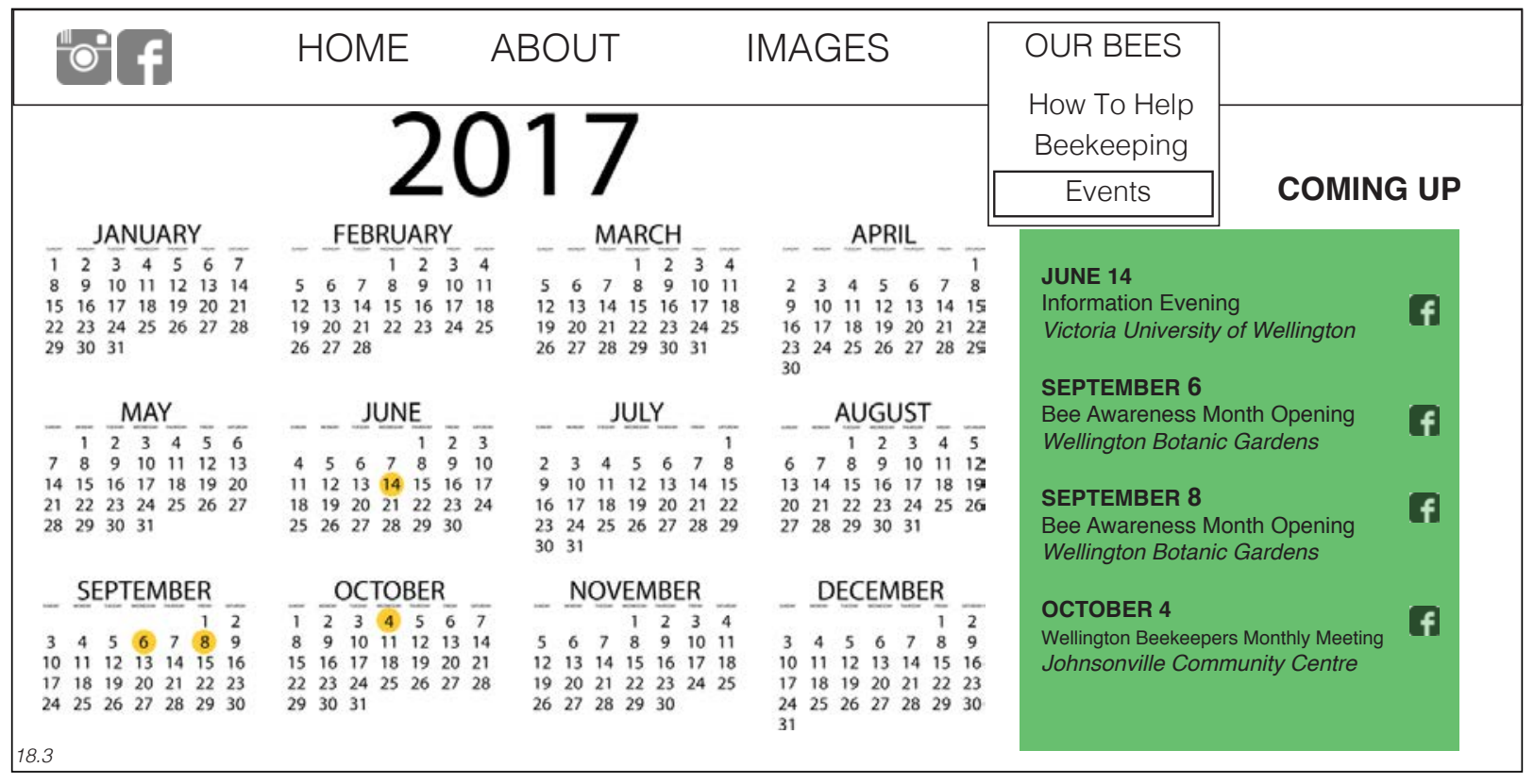

Figure 18.1 image by Author, Figures 18.2 sourced from Wellington Beekeepers Association, Figure 18.3 image from Google 


\title{
Wellington Beekeepers Club Hives
}

\author{
Chartwell, Wellington
}

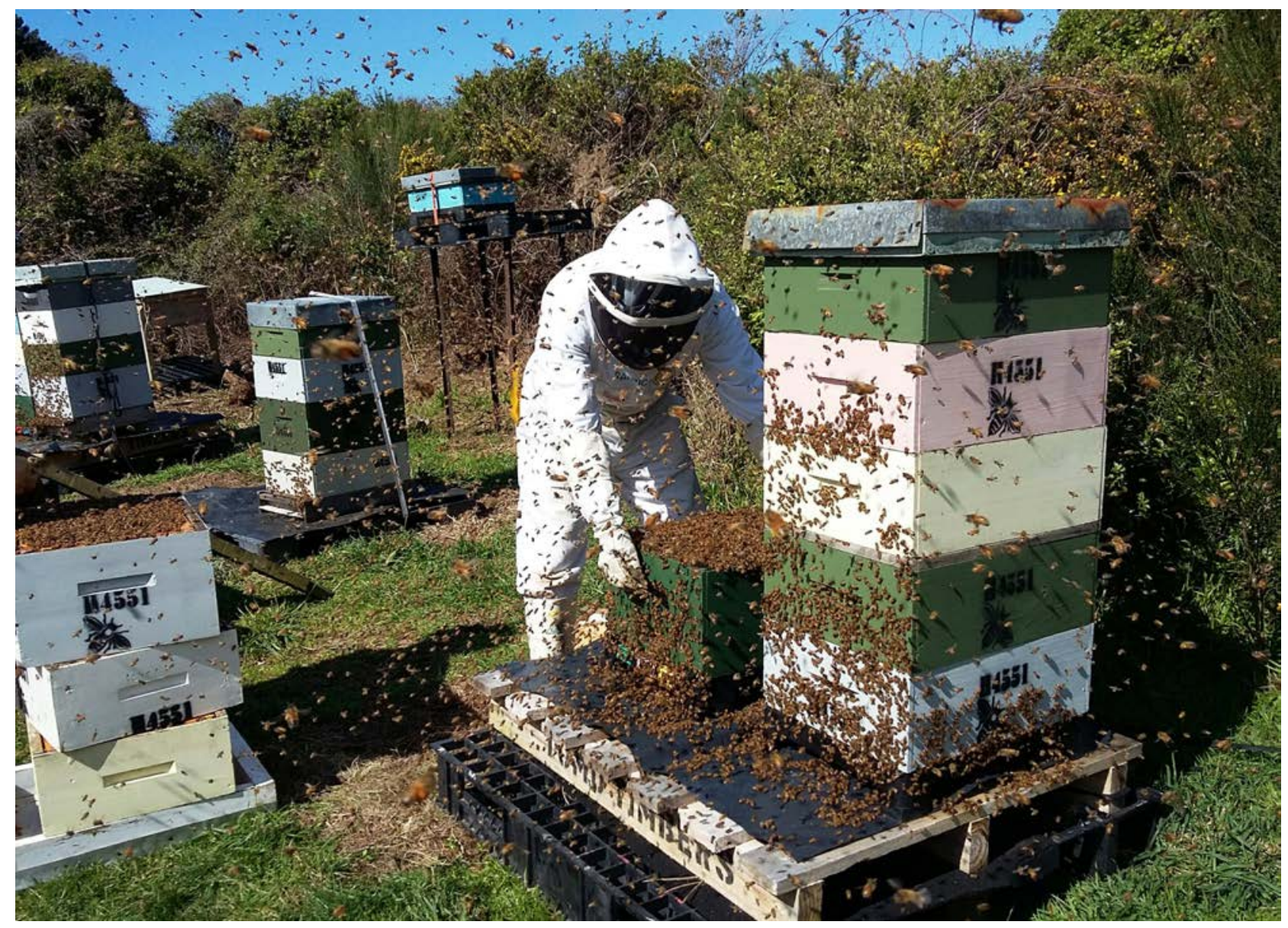

Figure 19. Club Beehive Location at Chartwell, by Wellington Beekeepers Association

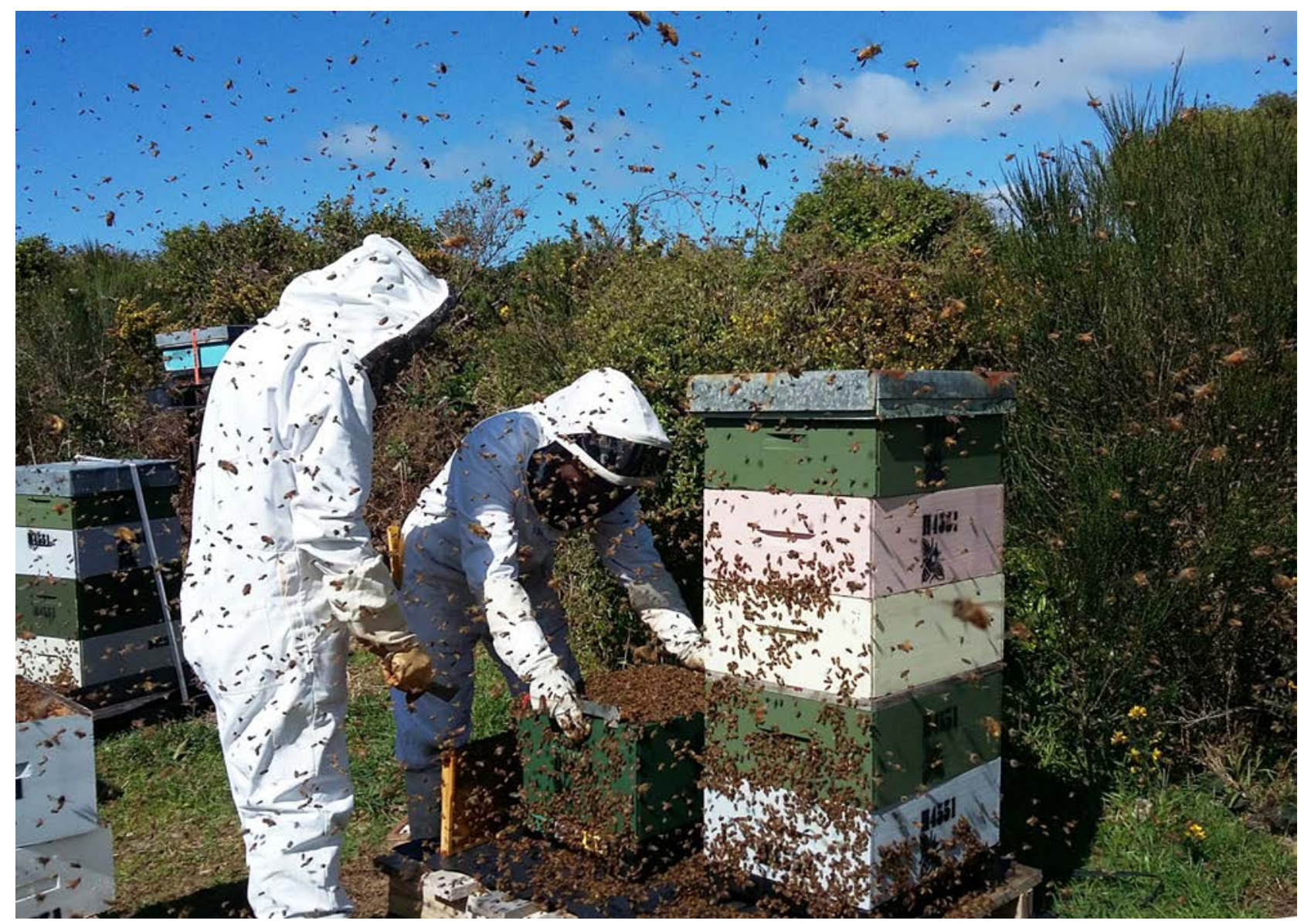

Figure 20. Moving Beehive Boxes at Chartwell, by Wellington Beekeepers Association 


\section{Physical Locations}

As a concept to link the physical experience with bee to an online interaction, QR codes would be placed at public hive sites like the beehives at the Wellington Botanic Gardens. This QR code would directly link audiences to more online content with the ability to also link images through location tagging on social media. The code would be displayed on current signage located at these sites, which already has existing bee information, to add a technological aspect to the experience. This design addon encourages further action to be taken to find out more on bees in Wellington and also what we can do to help their current situation. The image sharing on social media also links to the photographic/imagery output of my thesis, as it is a universal method which anyone can take part in. It becomes a very visual way to interact and interpret information as well as communicating across online platforms with the purpose to make it accessible for all audiences. This transfers the stimuli in the physical environment to online information and activity, with the option of sharing information in a variety of written and visual ways.

Communication design outputs include multiple design mediums that convey messages, information and education especially for public audiences. Design for the Education of the Public is an important Communication Design practise that streams information to larger audiences, and reinforces actions and behaviours. This is why I have chosen to use this visual interaction both in physical and online environments, to ensure communication and sharing among interested participants is possible and facilitated by my design concept. Realised in the design methodology, technology/social media activity is more common among younger audiences and is ideal for the target market of my design. The main target market includes local student populations and young professionals who may be temporarily or permanently located in central Wellington. Beehive locations at the Zoo, Wellington Botanic Gardens, and Community Gardens are publically accessible and are busy during the warmer seasons. Social media platforms attached the the online platform include Instagram and Facebook, as these are the largest, most frequently used applications with Instagram having a particular focus on image sharing. A collection of images posted on Facebook and Instagram will be collated on the platform through location tagging and specific hashtags. The sharing and curation of public images is a main component in the design as it allows external input to generate and update content and visuals, alongside existing images of my own. The aim is to translate community involvement into an online environment where information and communication tools can easily be reached and shared by individuals. 


\section{Wellington Botanical Gardens}

Beehive No.2 (behind herb garden)

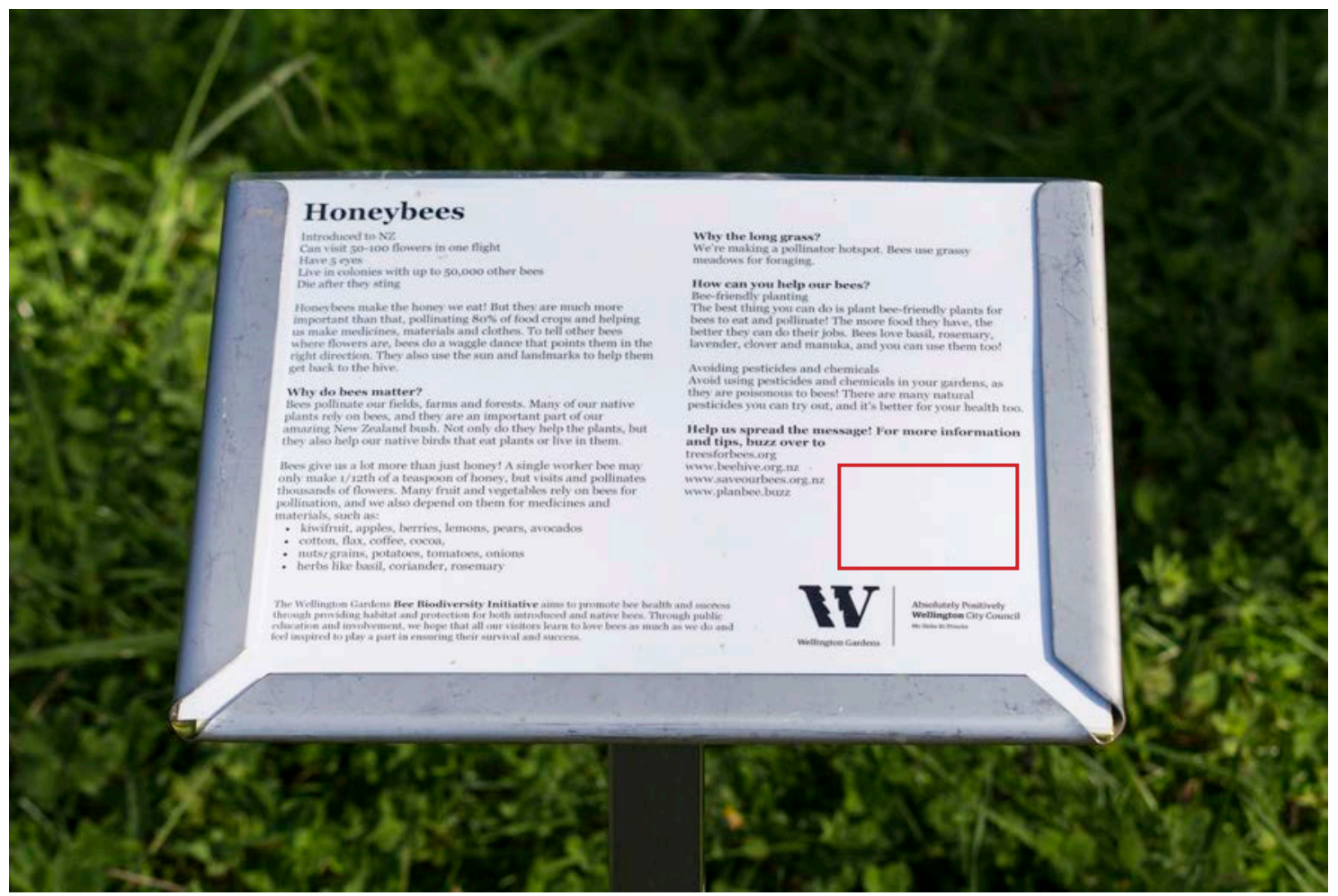

Figure 21. Signage and Information at Wellington Gardens Beehive Location, by Author

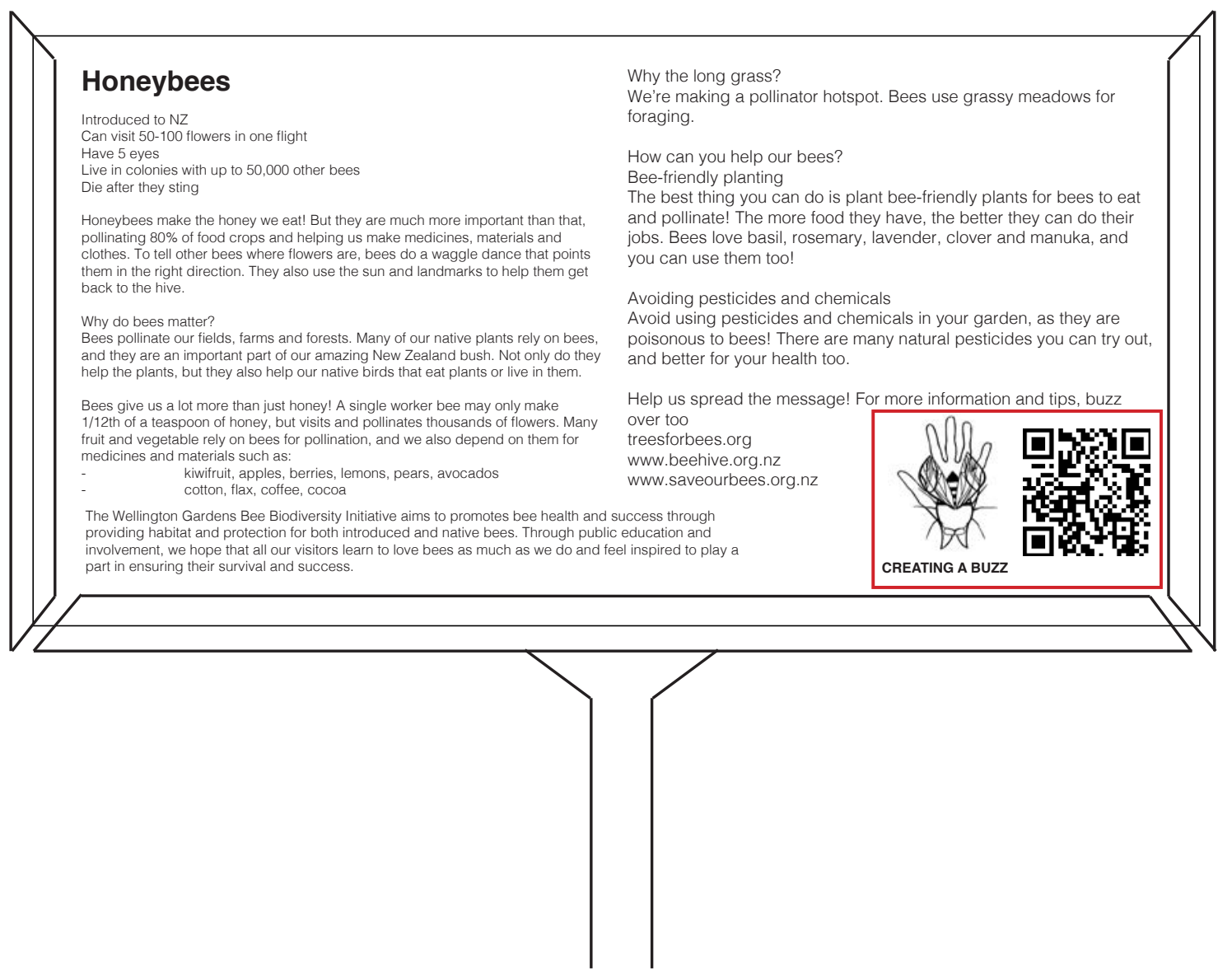




\section{Social Media Posts}

\section{Linked to Website}

\section{우 7}

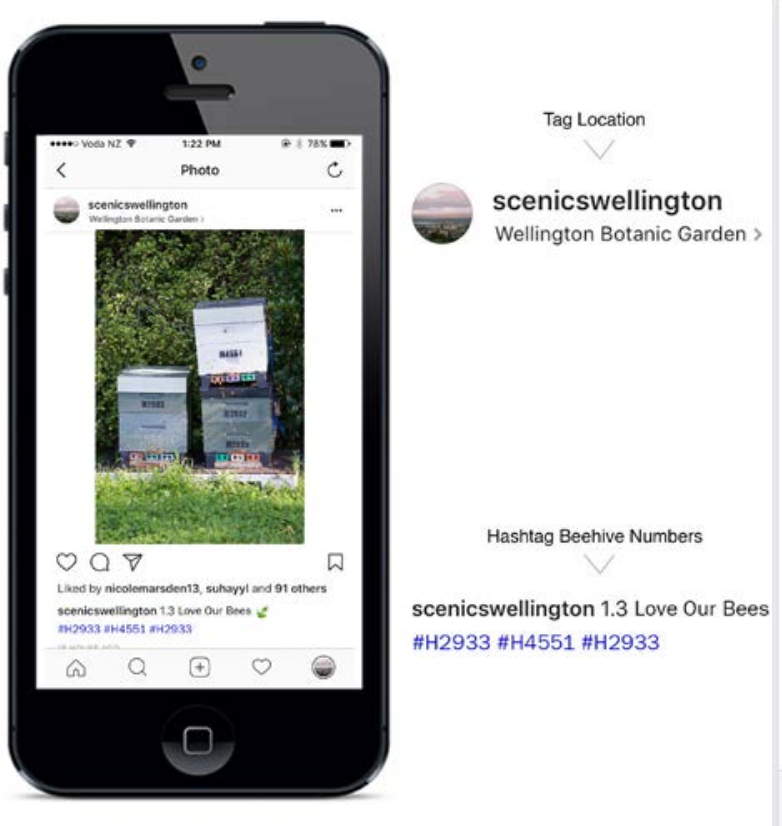

Figure 22. Instagram Post Example, by Author
FPy Madz Cooper at 9 Wellington Botanic Garden.

1 of 10,000

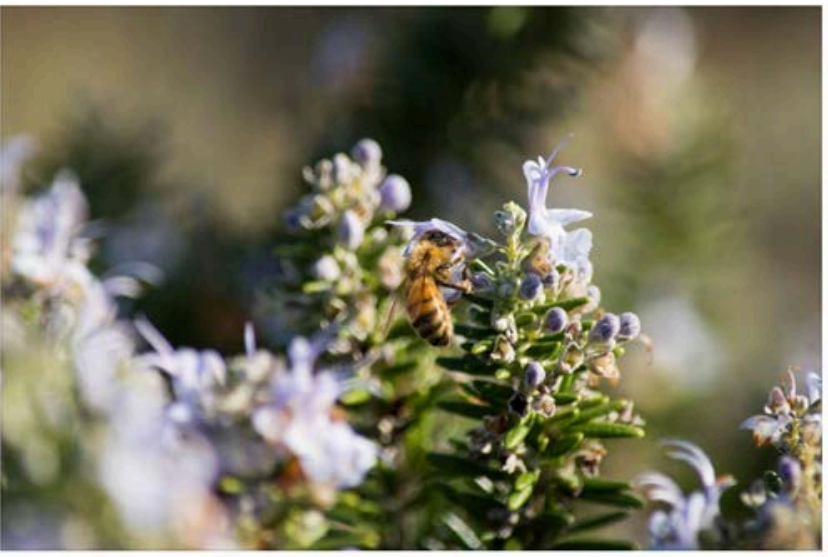

Like Comment

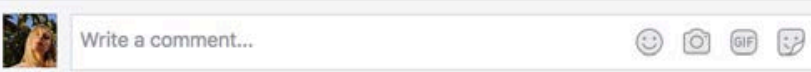

Figure 23. Facebook Post Example, by Author

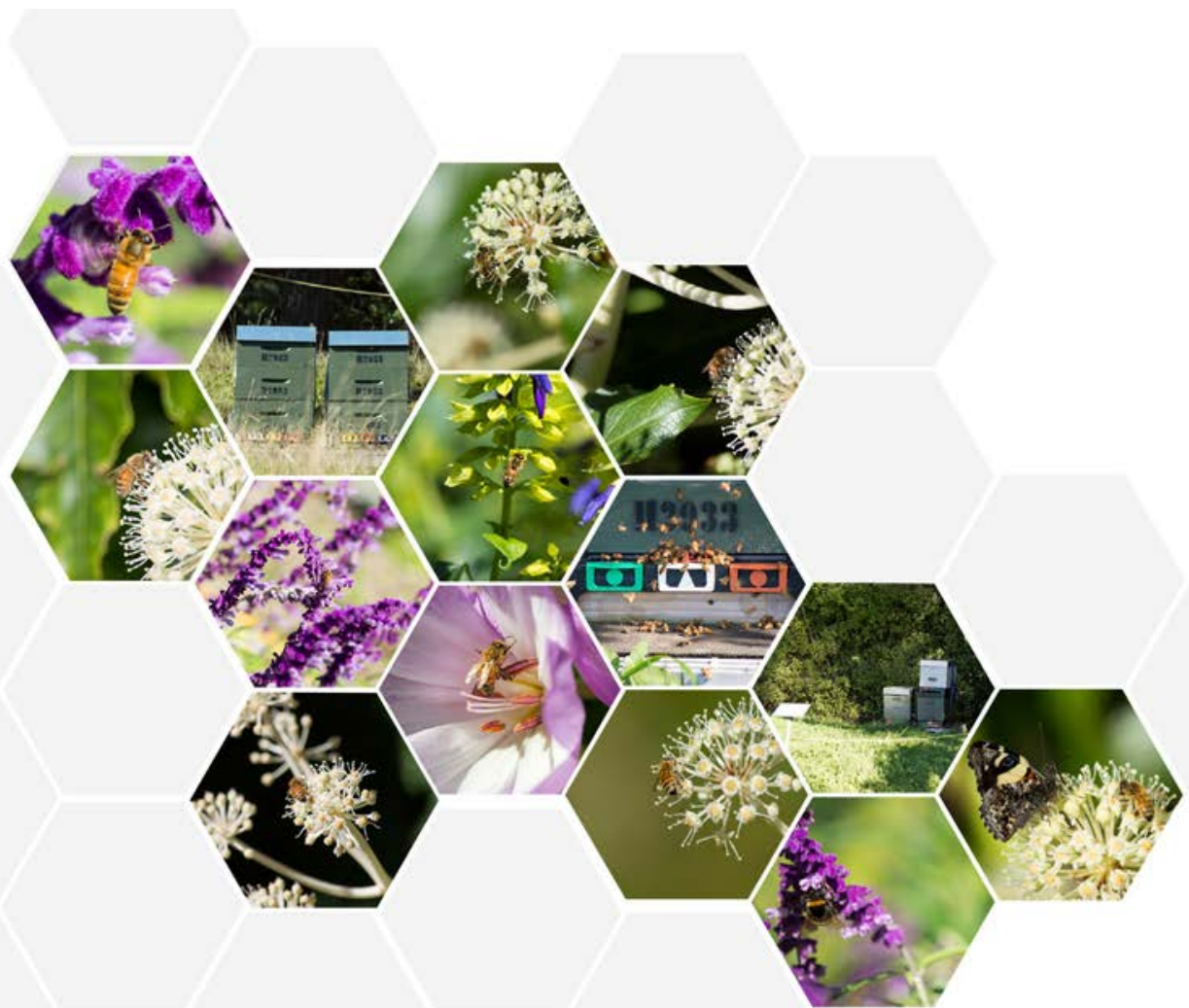

Figure 24. Image Collection Graphic of Social Media Posts on Website, by Author 


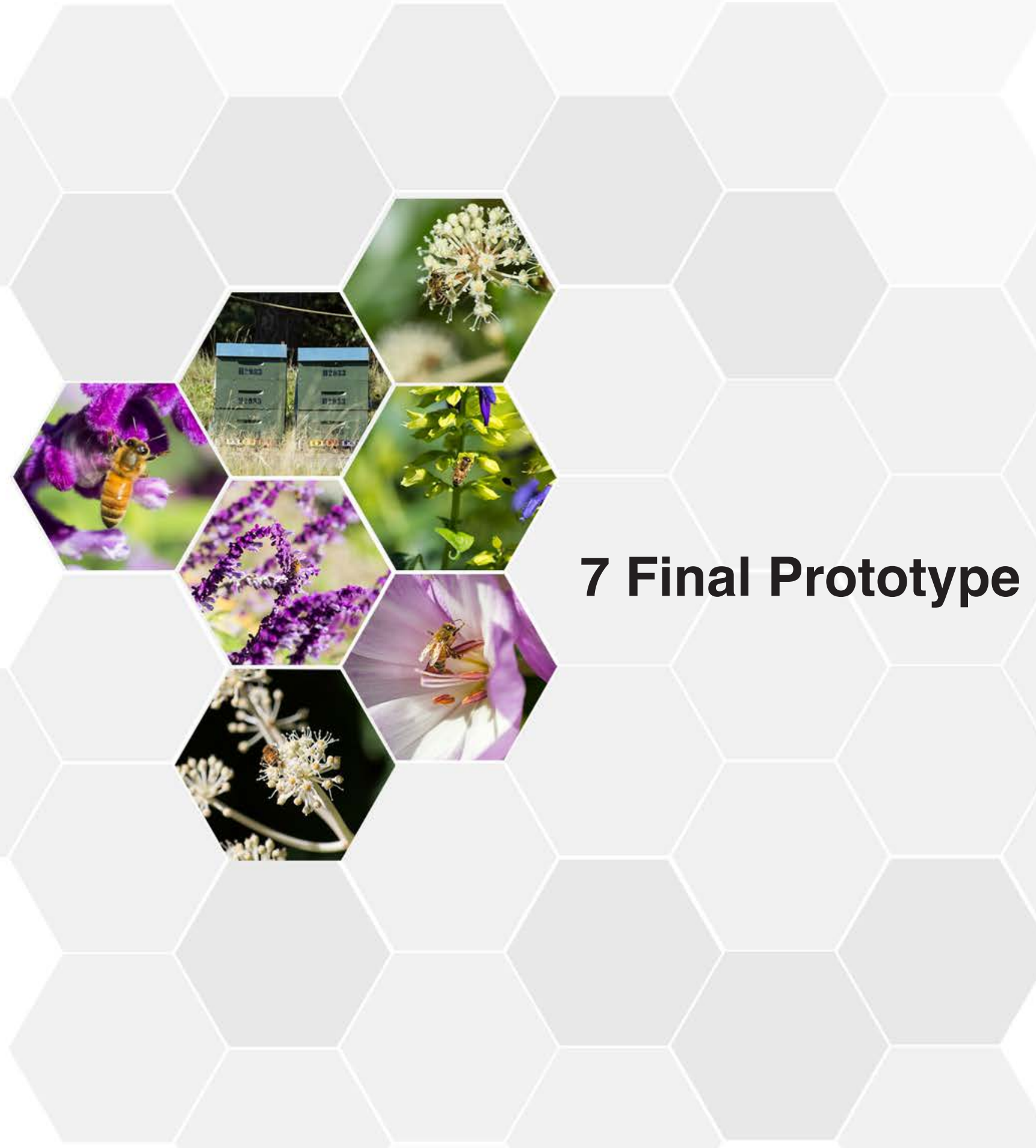




\section{Home Page}

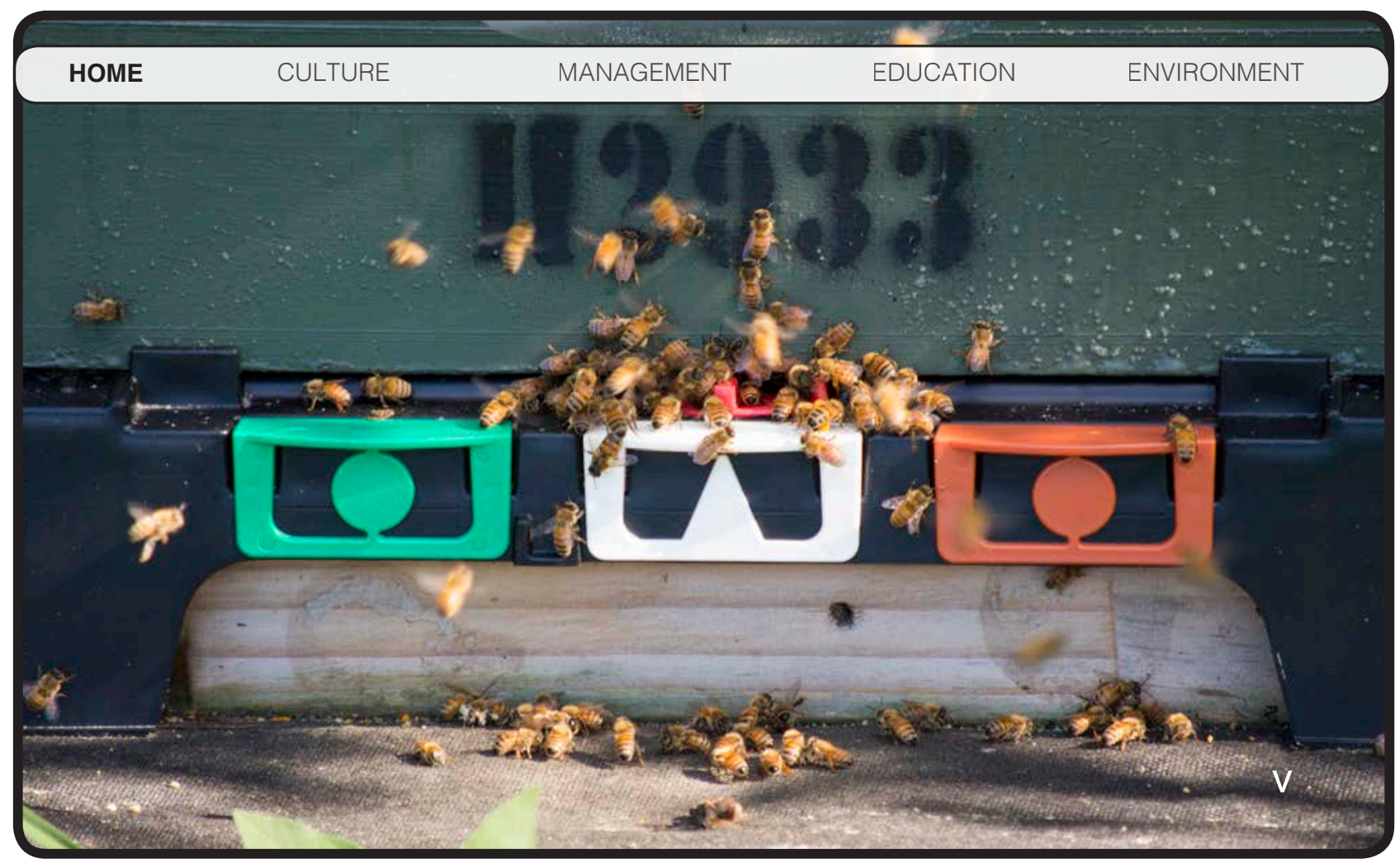




\section{Social Media Pages}

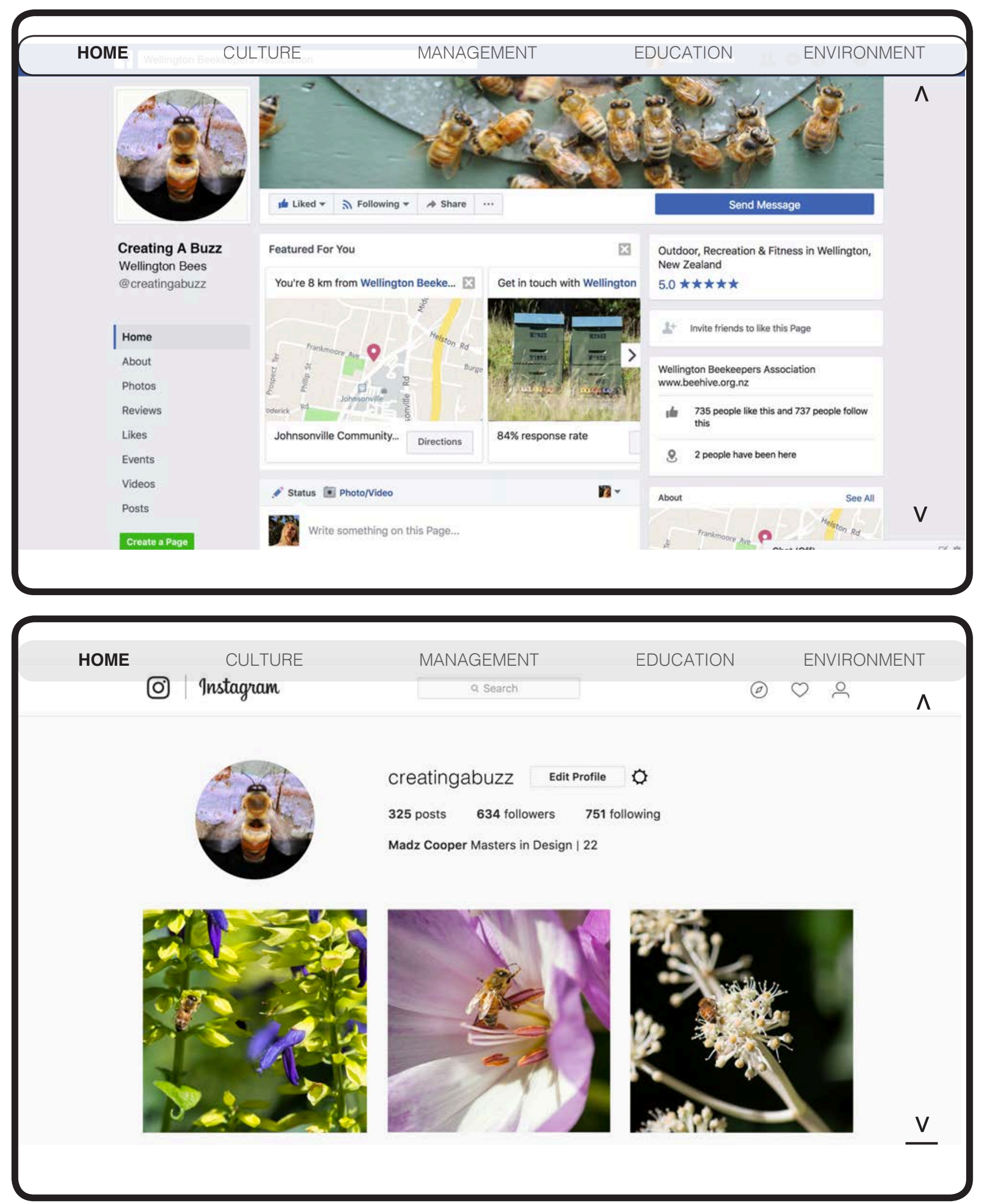




\section{Culture}

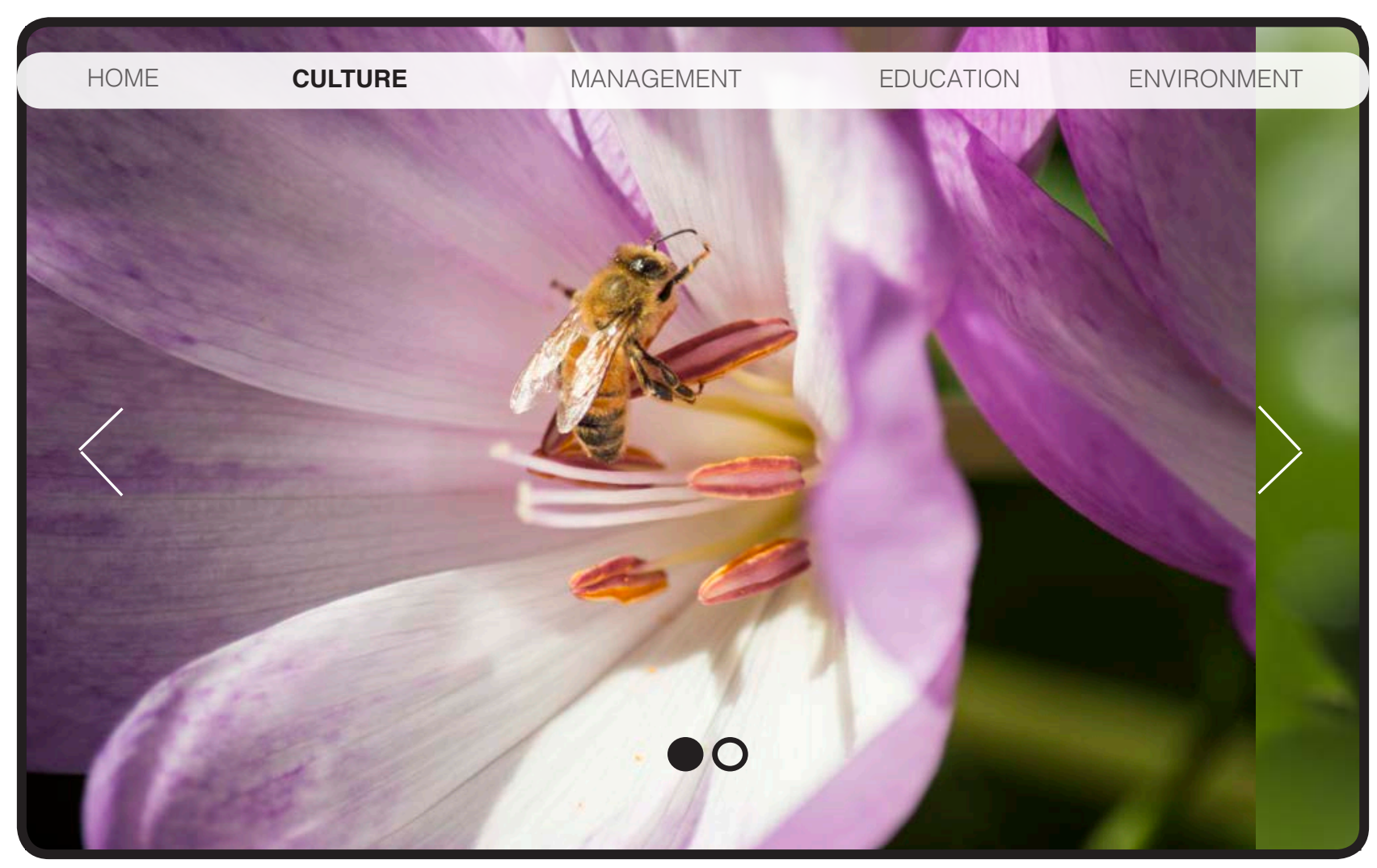

\section{Cultural Associations}

The cultural association of New Zealand's honey bee industry, especially large volumes of Manuka plantations relates to the well-being and use of our land, with native flora relating directly back to our people. Showing the physical interaction of the bee with a native flower illustrates these typical relationships within our local ecosystems.

\section{Honeybee Purpose}

The primary purpose of the honeybee is the pollination process, which involves collecting nectar sources from flowering plants and returning the nectar to the hive to make honey. 

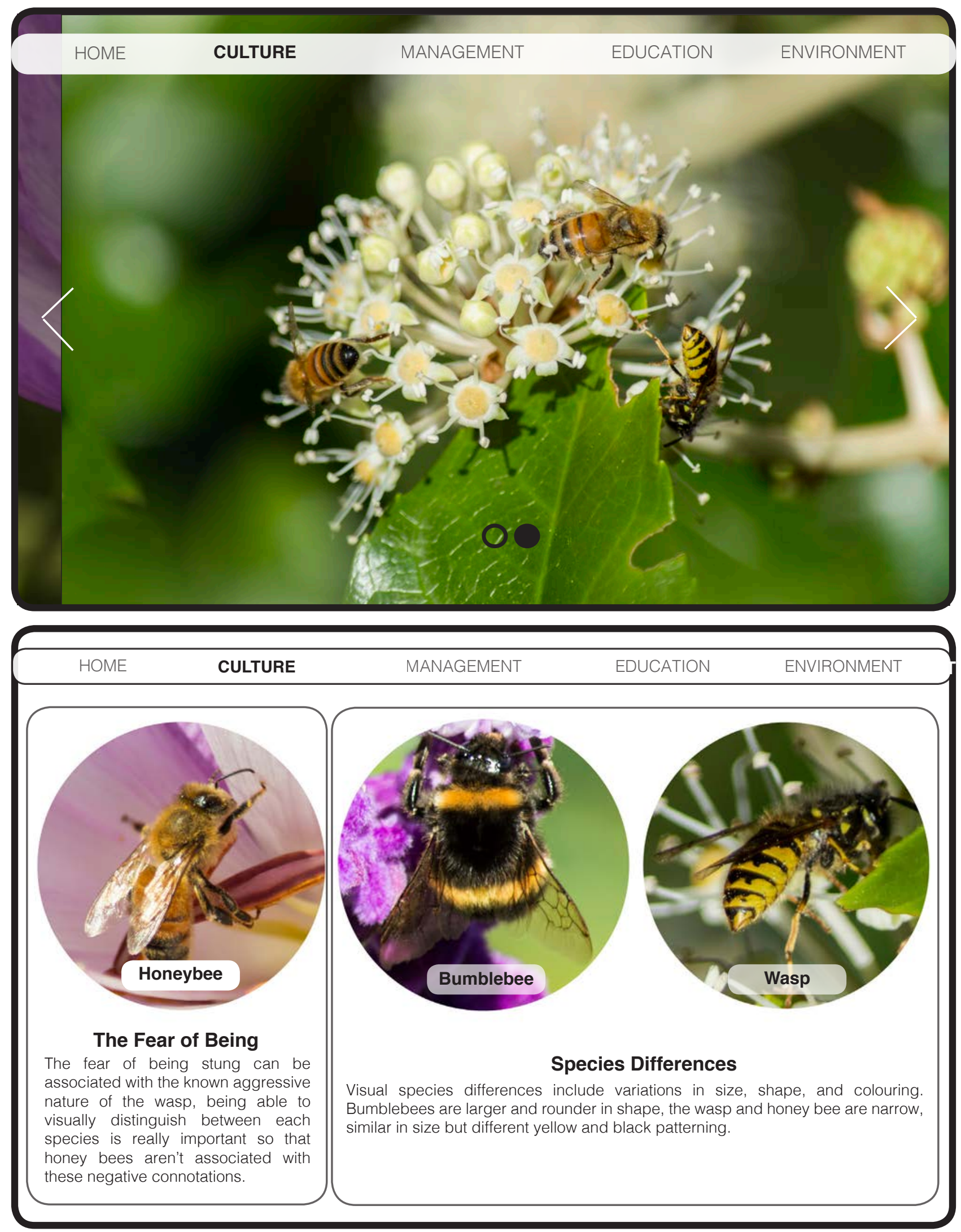


\section{Culture}

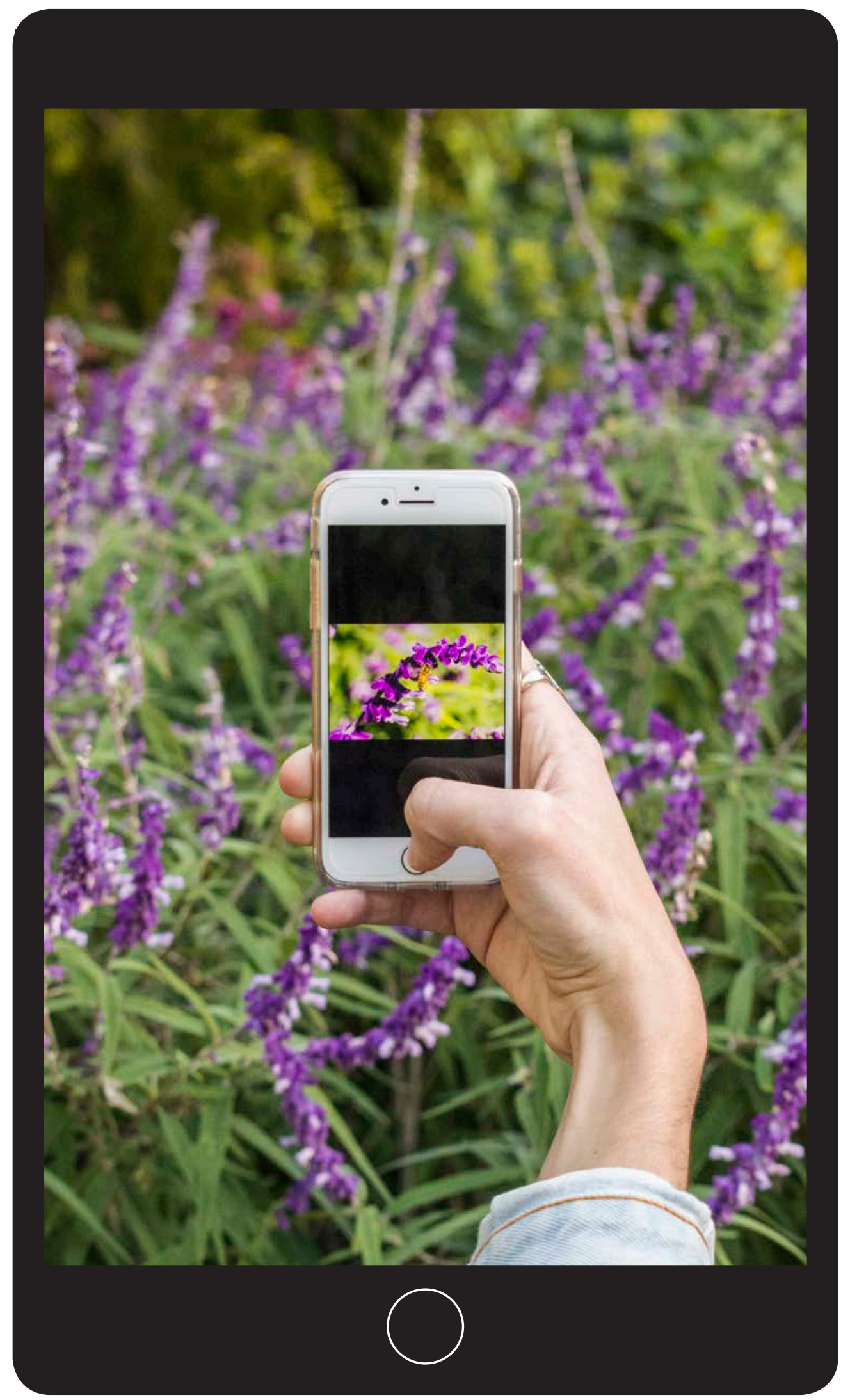

\section{Image Sharing}

The action of image taking and sharing from physical beehive locations in Wellington is important as it visually communicates and reinforces positive interactions and experiences with bees. These images also contextualise the experience within a Wellington setting, and makes others aware of what's currently happening in our surroundings. 

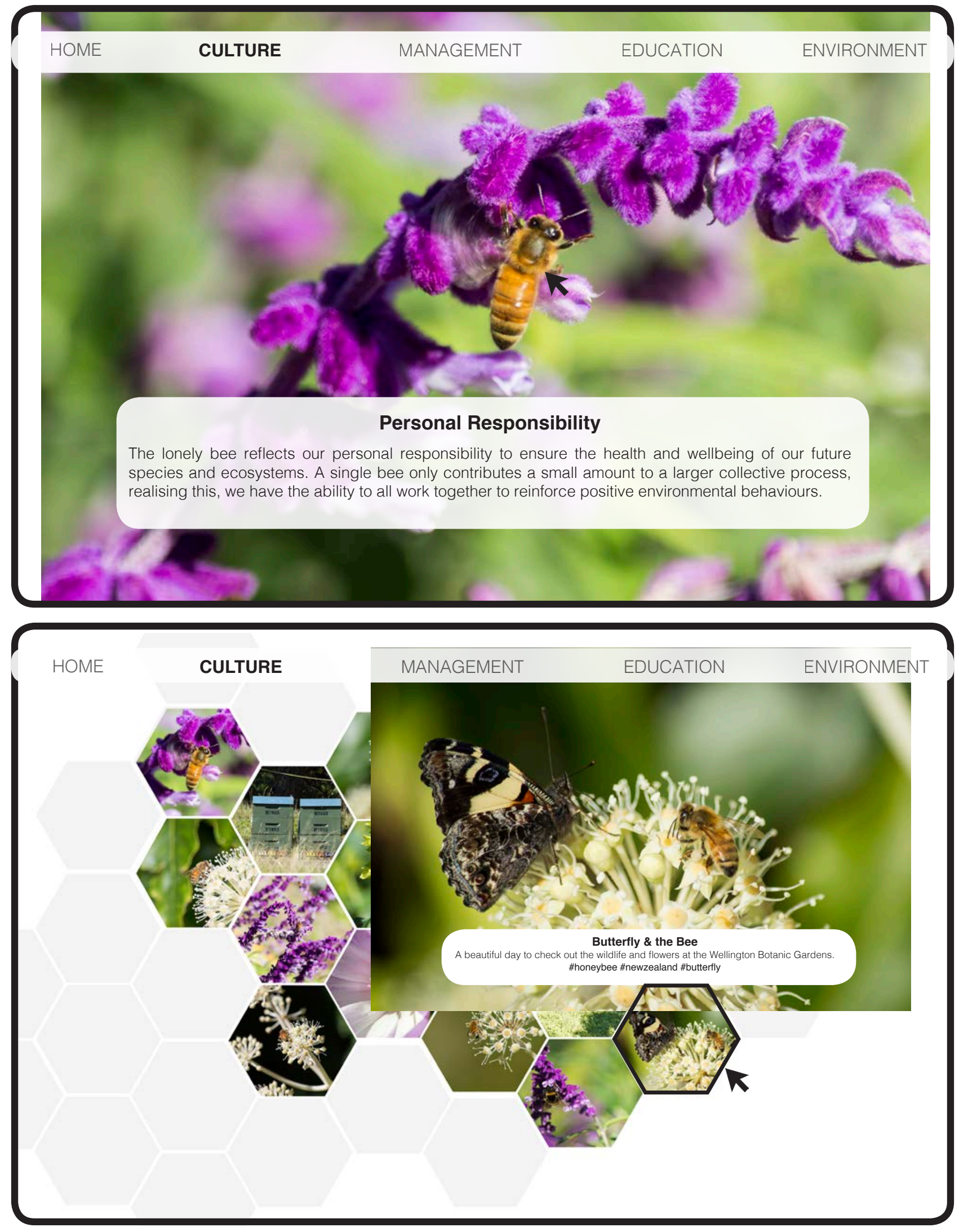

\section{Community Involvement}

This shows an archive of images taken at different bee location in Wellington, with input from Facebook and Instagram and a direct input into the online platform. The relates to the involvement needed in physical communities by encouraging online interactions and the idea of an online community being visually representative of physical individual experiences. 


\section{Management}
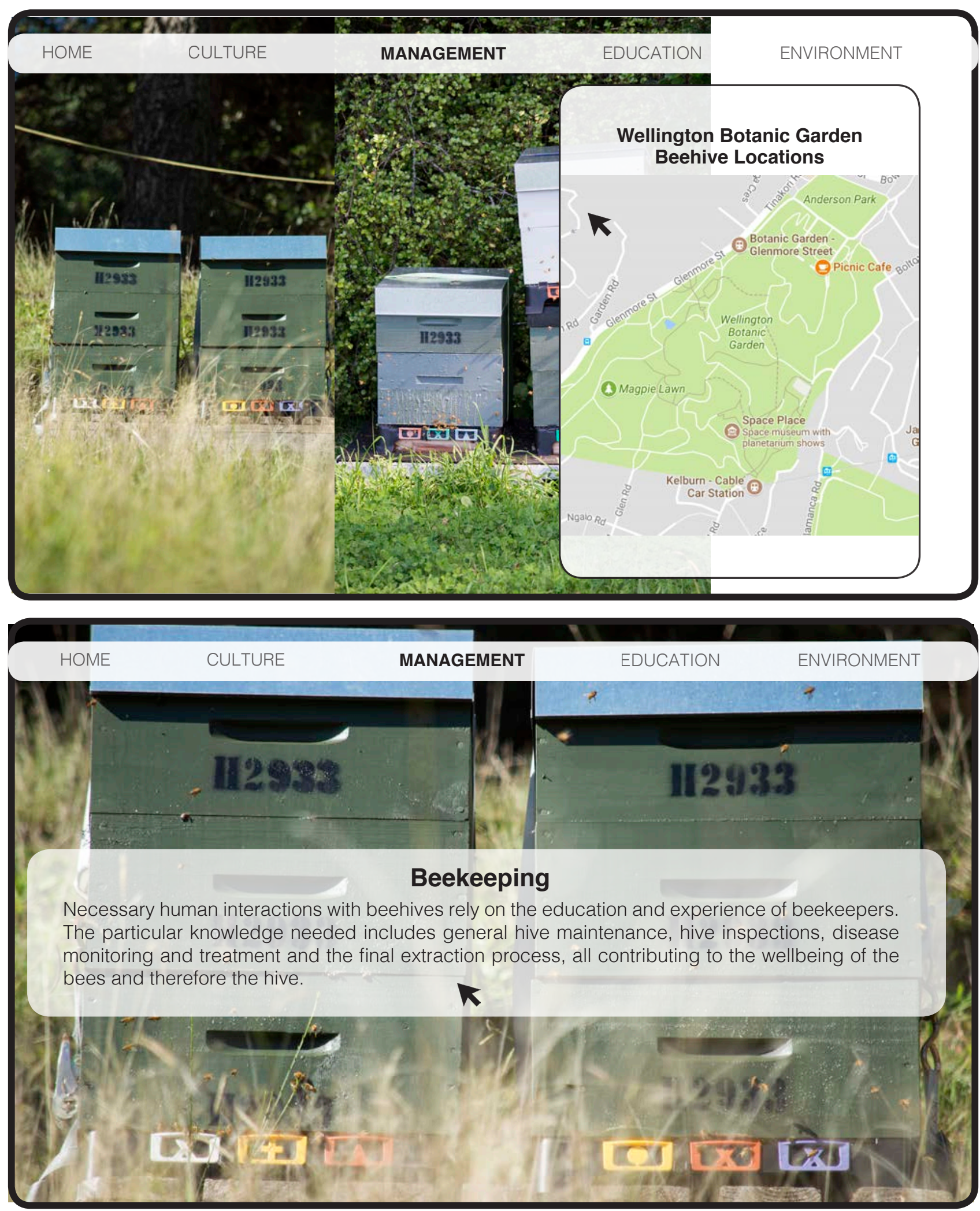

\section{Overpopulation}

With the beekeeping trend having a recent surge in popularity, the carrying capacity of environments needs to be considered. Larger numbers of urban hives and beekeepers can lead to over populated and potentially poorly managed hives. 


\section{Education}
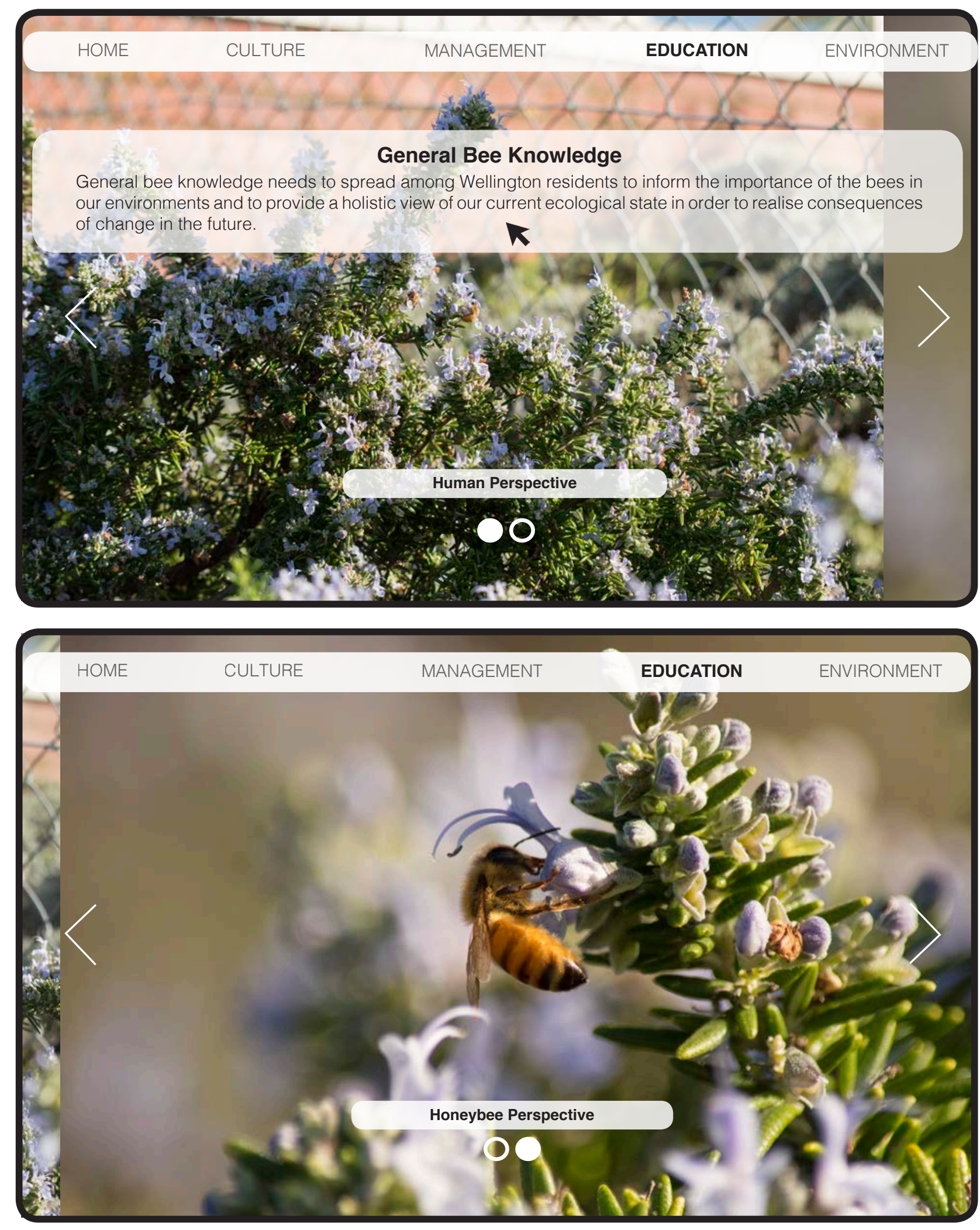

\section{Food Sources}

The availability of food (nectar sources) relies on the seasonal flowering of crops, as there needs to be a plentiful supply to support the populations of bees in the area. The spraying of harmful pesticides also needs to be educated, as chemicals are transferred from the crop to the bee, killing the bee. 


\section{Environment}

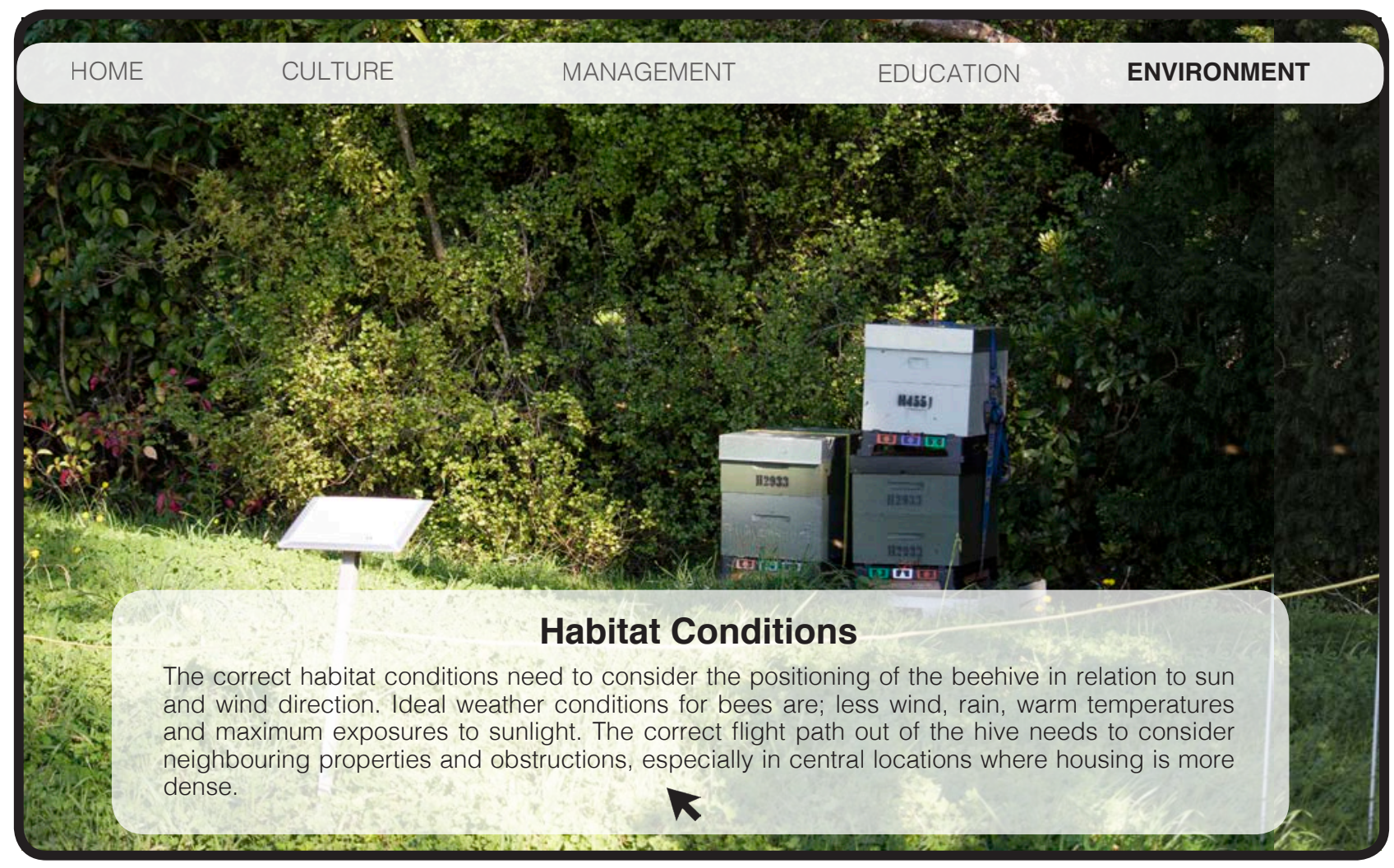

\section{Honeybees}

Introduced to NZ

Can visit 50-100 flowers in one flight

Have 5 eyes
Live in colonies with up to 50,000 other bees

Die after they sting

Honeybees make the honey we eat! But they are much more important than that, pollinating $80 \%$ of food crops and helping us make medicines, materials and clothes. To tell other They also use the sun and landmarks to help them get back to the hive.

Why do bees matter?

Bees pollinate our fields, farms and forests. Many of our native plants rely on bees, and they are an important part of our amazing New Zealand bush. Not only do they help the plants, but they also help our native birds that eat plants or live in them.

Bees give us a lot more than just honey! A single worker bee may only make 1/12th of a teaspoon or honey, but vists and pollinates thousands of llowers. Many frut and vegetable rely on
materials such as:

-kiwifruit, apples, berries, lemons, pears, avocados

cotton, flax, coffee, cocoa

- nuts, grains, potatoes, tomatoes, onion

The Wellington Gardens Bee Biodiversity Initiative aims to promotes bee health and success through providing habitat and protection for both introduced and native bees. Through public education and involvement, we hope that all our visitors learn to love bees as much as we do and feel inspired to play a part in ensuring their survival and success
Why the long grass?

We're making a pollinator hotspot. Bees use grassy meadows for foraging.

How can you help our bees?

Bee-friendly planting

The best thing you can do is plant bee-friendly plants for bees to eat and pollinate! The more food they have, the better they can do their jobs. Bees ove basil, rosemary, lavender, clover and manuka, and you can use them too!

Avoiding pesticides and chemicals

Avoid using pesticides and chemicals in your garden, as they are poisonous to bees! There are many natural pesticides you can try out, and better for your health too.

Help us spread the message! For more information and tips, buzz over too treesforbees.org

www. beehive.org.nz

www.saveourbees.org.nz

www. planbee.buzz

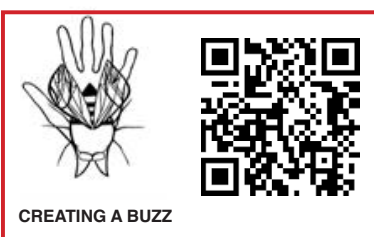




\section{Application of Communication Design}

Each collection of images addresses the key themes realised in the stakeholder analysis culture, management, education and the environment. Referring back to Jorge Frascara's (1997) communication design principles, these images "translate the invisible into the visible" and the visual communications are "aimed at broadcasting specific messages to specific sectors of the public" (p.2). Design for Information and Design for the Education of the Public were the primary communication practices used, where the work of visual designers moves between informative and persuasive avenues, with the final design combining multiple avenues of information for intended audiences. The intended purpose of education aims to encourage positive sustainable behaviours through active participation and interaction with these visual materials, extending across linked social media platforms. Frascara believes that education cannot be reduced to the transmission of information, in response to this I chose to design engaging visuals and social media platforms to reinforce new technological ways of learning that can be applied to current and future physical and online contexts. The combination of a public beehive location and online information allows people to first immerse themselves in the physical context and then learn more through the supportive online material and images.

Throughout the research process I have identified key information about Wellington's current honey bee populations and have formatted communication design tools. This allows for written information to be represented through visuals, and for it to become an applied universal method of communication. The final spread combines visuals to tell a story about the perception of the bee and current issues that need addressing to ensure the future health and wellbeing of our vital species. It also aims to create an empathic relationship between humans and bees by viewing the world at a much larger scale, one that would be seen by the bee itself. The details of connections through a QR code at public locations reinforces the physical and online relationship of experiences and information by adding a technological aspect. These social media interactions are specifically targeted at the intended audience of students and young professionals, but is also applicable to other Wellington audiences. 

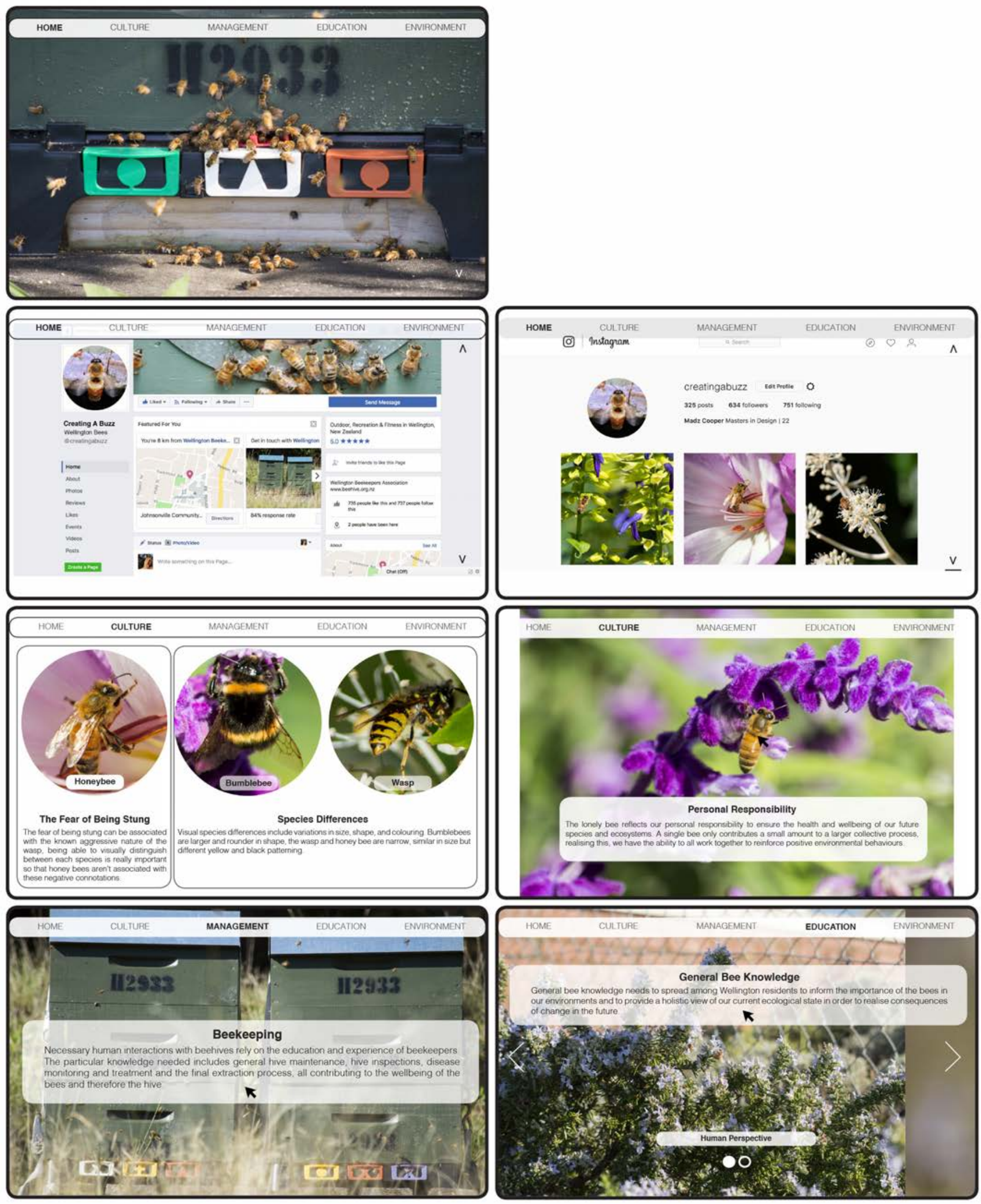

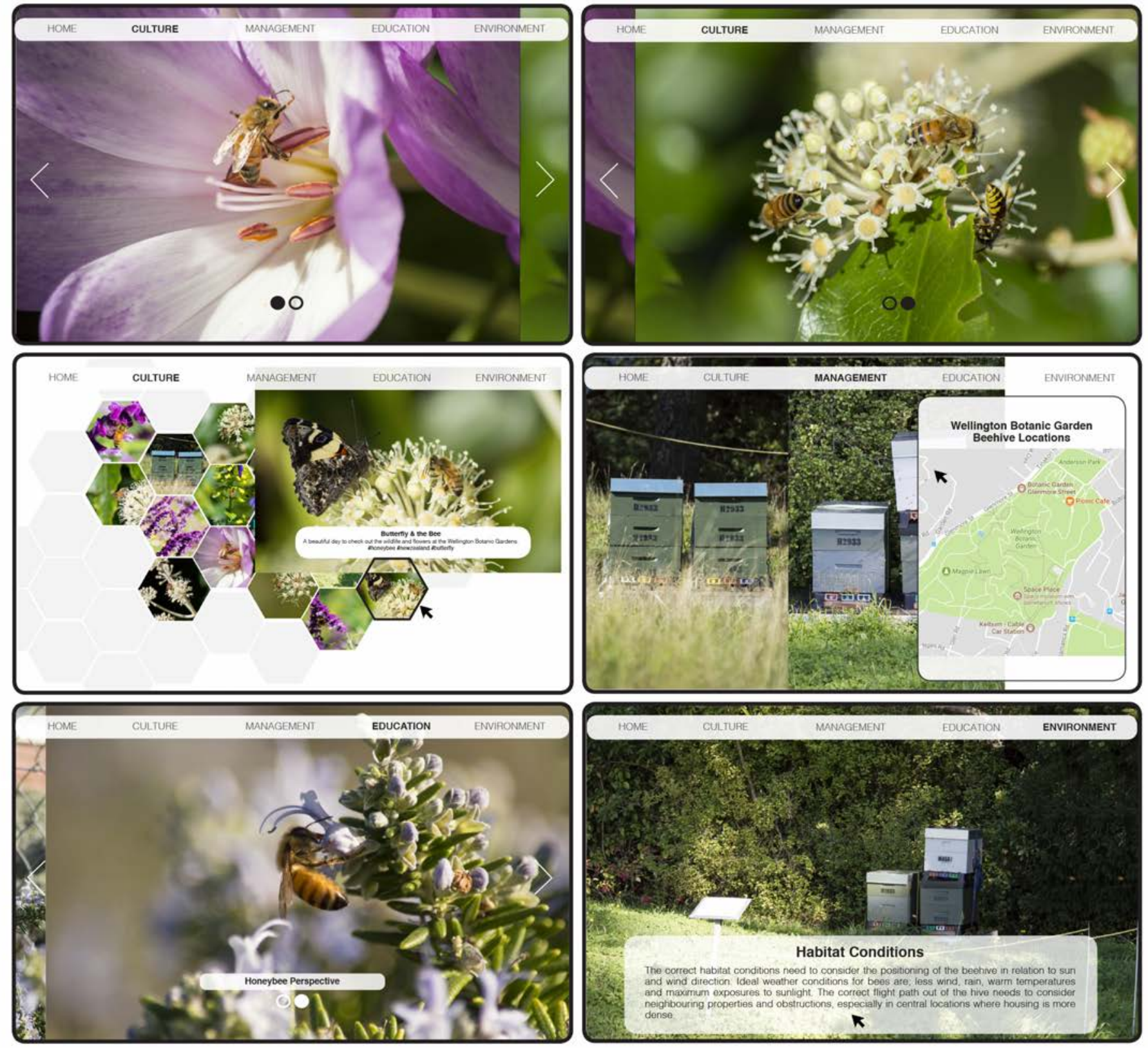


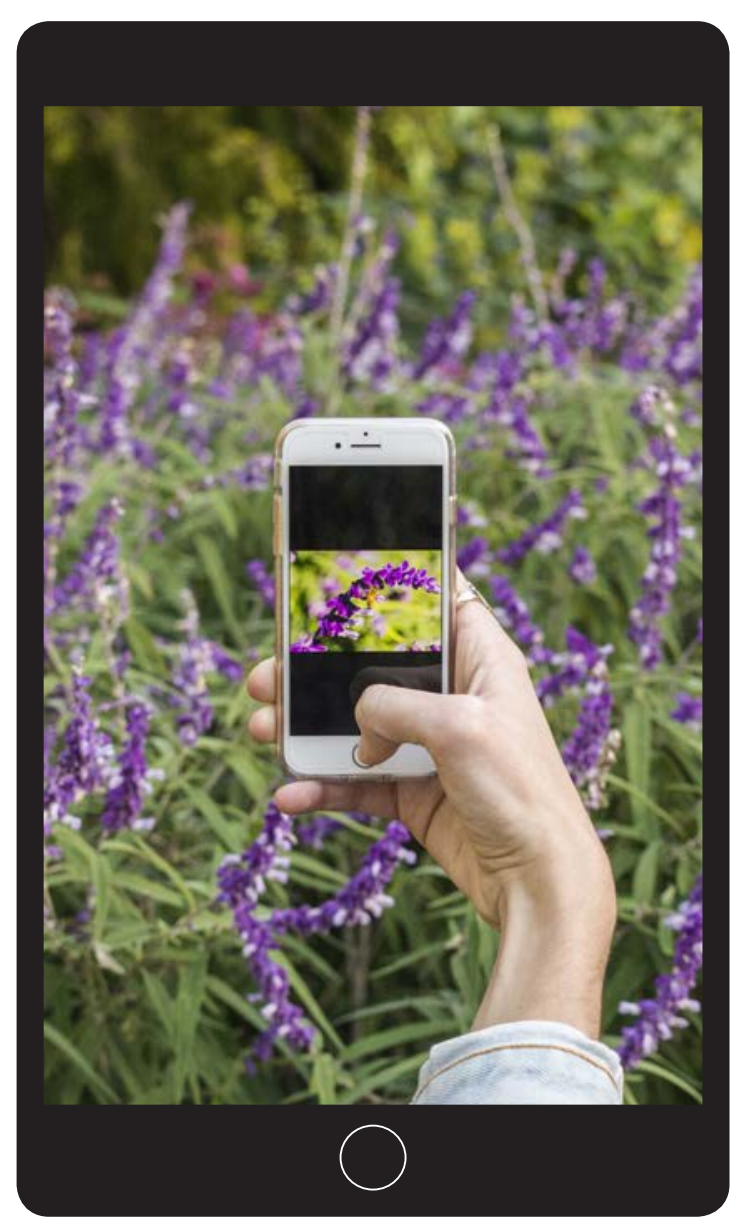

\section{Honeybees}

Introduced to NZ

Can visit 50-100 flowers in one flight

Have 5 eyes

Live in colonies with up to 50,000 other bees

Die after they sting

Honeybees make the honey we eat! But they are much more important than that, pollinating $80 \%$ of food crops and helping us make medicines, materials and clothes. To tell other

Why do bees matter?

Bees pollinate our fields, farms and forests. Many of our native plants rely on bees, and they are an important part of our amazing New Zealand bush. Not only do they help the plants, but they also help our native birds that eat plants or live in them.

Bees give us a lot more than just honey! A single worker bee may only make 1/12th of a teaspoon of honey, but visits and pollinates thousands of flowers. Many frutt and vegetable rely on bees for pollination, and we also depend on them for medicines and materials such as:
- kiwifruit, apples,

- kiwifruit, apples, berries, lemons, pears, avocados

- cotton, flax, coffee, cocoa

herbs like basil, coriander,

The Wellington Gardens Bee Biodiversity Initiative aims to promotes bee health and success through providing habitat and protection for both introduced and native bees. Through public education and involvement, we hope that all our vis.
We're making a pollinator hotspot. Bees use grassy meadows for foraging.

\section{How can you help our bees?}

Bee-friendly planting

The best thing you can do is plant bee-friendly plants for bees to eat and pollinate! The more food they have, the better they can do their jobs. Bees love basil, rosemary, lavender, clover and manuka, and you can use them too!

Avoiding pesticides and chemicals

Avoid using pesticides and chemicals in your garden, as they are poisonous to bees! There are many natural pesticides you can try out, and better for your health too.

Help us spread the message! For more information and tips, buzz over too treesforbees.org

www beehive.org.nz

www.saveourbees.org.nz

www. planbee.buzz
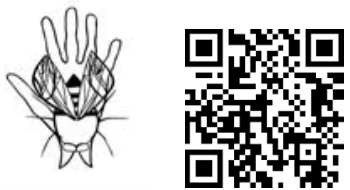

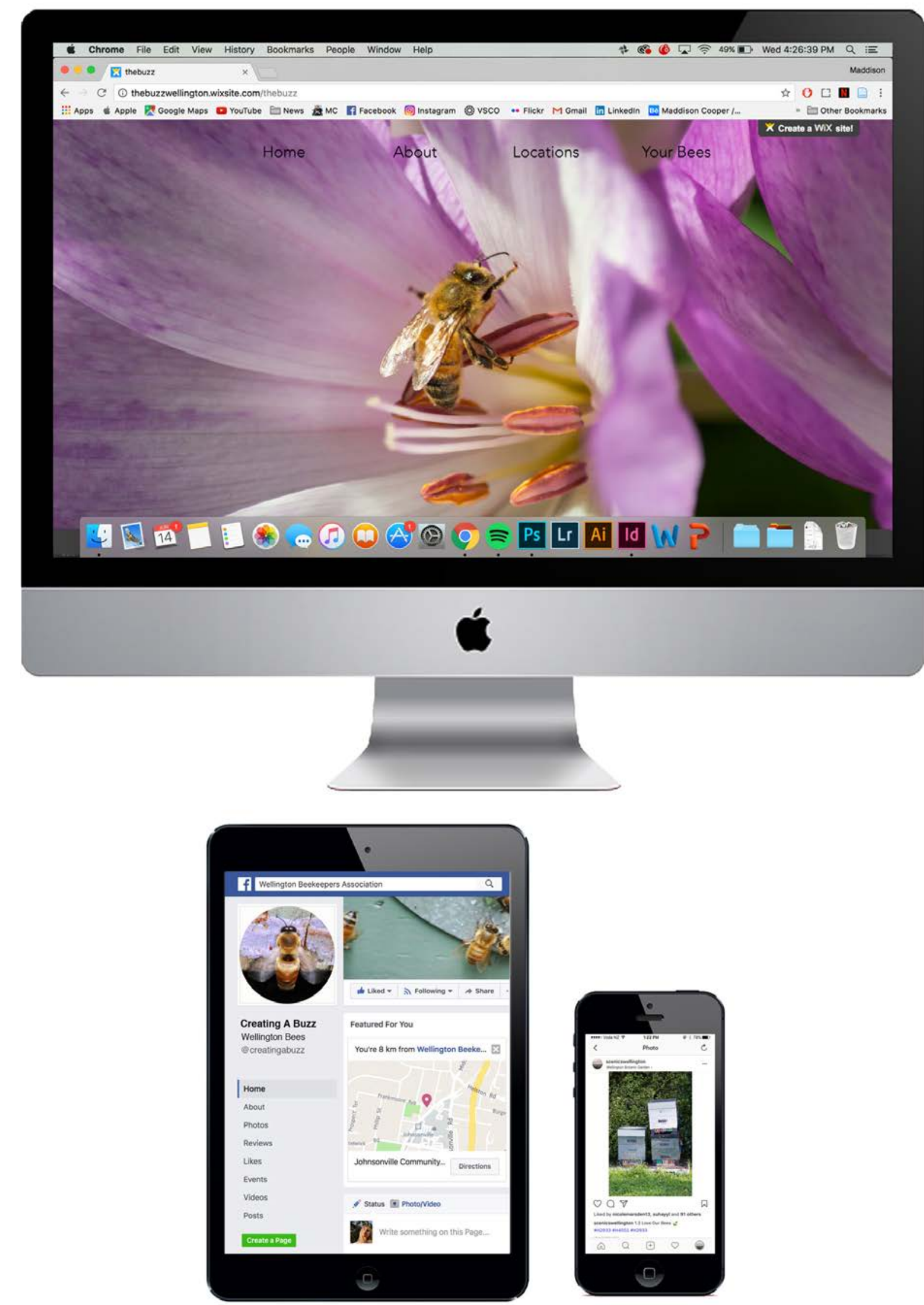


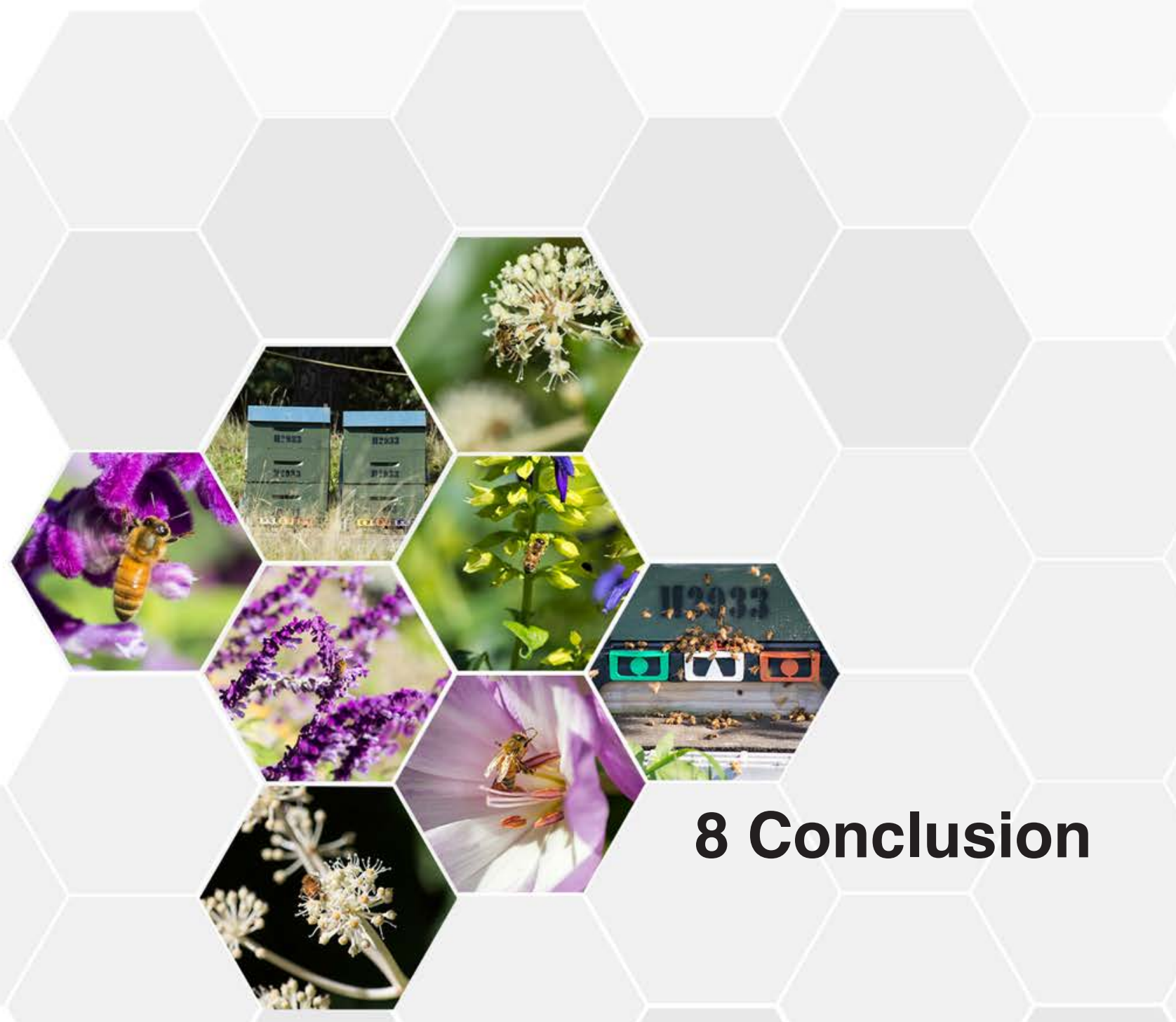




\section{Conclusion}

This thesis has used research and design in an investigation of key social, cultural and environmental concepts that impact Wellington's honey bee populations and beekeeping culture. This exploration highlights that the way we focus and distribute information needs to include meaningful education and knowledge of bee health and well-being, with the aim to sustain and increase our bee population numbers over time. The knowledge and education of our honey bees is imperative in raising awareness and creating empathic relationships in order to encourage better caretaking practices of our bee ecologies and surrounding ecosystems. The application of the Communication practice and Design for the Education of the Public, relies on the interpretation of information and the persuasive behaviour of written and visual content relevant to this particular issue. The research has driven the development of a communication design approach, which aims to raise awareness towards current barriers and concerns of Wellington's bee populations. The stakeholder perspectives and design recommendations chapter demonstrates an overall perception that issues concerning bees and beekeepers are becoming increasingly popular in Wellington. With the bee currently having applications to many different forms of products, education, research, and practices the scope for raising awareness needs to focus on Wellington's local condition. The cultural perception and the well-being of honey bees is important to address as they reflect New Zealand's culture surrounding our bee industry and how human actions and behaviours relate back to our people and the future sustainability of our land.

These current barriers and concerns in Wellington include the cultural perception of the bee and bee industry, management of hives, the education of the bee and their essential purpose, and habitat and environmental sustainability. By encouraging immersive interactions at public beehive locations, our local bees can be viewed along with supplementary online visual and written material that reinforces these experiences and provides an educational takeaway. This is where the integration of a QR code can link physical and online information, as well as providing links to social media channels, with viewing and sharing being a specific interaction that can aid in raising awareness. This visual education tool is formulated around current industry and expert understandings with specific content being supplied by key stakeholders and refined into a design prototype. This tool indicates a template and design process that can be used to address other critical issues that are related to a specific context, purpose and audience. The information and visual content aims to be applied to different online platforms, allowing for viewing on different devices such as computers, tablets and mobile phones. The process of combining content with the function of the social media applications and online platforms was essential in the application of the design to it's intended audience. Extensions for this design could include using these graphics and written content on a functioning website, which would be permanently available to all online audiences. With a particular focus on technological avenues in this thesis, future advancements could consider other types of information sharing and interaction design, linking physical and online bee environments.

As this thesis includes the perspectives of a small number of stakeholders, the information used is a holistic overview of current bee trends in Wellington. Through my interpretation of these ideas I believe that current fears towards bees can be reinterpreted through the visual communication mediums of photographs and graphics. The relationships between native ecosystems are important to New Zealand's culture, with native flora and biodiversity benefiting from the pollination of bees. To enhance these relationships and communication of these ideas, photographic processes and visual outputs can be used and distributed through multiple media channels. Photographic mediums accentuate different scales and details of the bee and creates a visual insight into their daily lives and physical beauty. The willingness to relate to more-than-human species can be fostered by visual recognition (photography) and encourage a want to share experiences and interactions online. Larger impacting concepts of sustainability and local environments are persuaded through written and visual information, with particular instructions focusing on practices that will help in fostering the growth of honey bee populations. The necessary practices of beekeeping impacts the wellbeing of hives, with correct maintenance and disease management being imperative to their survival. The initial education of beekeepers is supported through Wellington's Beekeeping Association, but there needs to be a want for new beekeepers to be patient with their learning and understand the basics before dedicating themselves to setting up a beehive. 
In addition to participating in beekeeping practices, the public can support and care for honey bees without investing themselves as beekeepers. These activities can include individual actions through to community based projects that have the potential for people to encounter bees and to be empathetic of their existence and purpose. Opportunities for personal experiences are made possible by public beehives such as those positioned in the Wellington Botanic Gardens, The Zoo and in suburban community gardens. With the potential to rent and host hives, plant bee friendly flowers and aid in spreading education and knowledge, our communities can work together to build a resilient plan for the future sustainability of our environments. As there is already a plentiful supply of online resources available to New Zealanders, bee interactions and education tools can be extended through research and communication design.

\section{Summary of Chapters}

The literature and design reviews focus on current academic literature and designs that aim to reinforce the validity and scope of this topic. A particular urban Wellington context was identified, relating to the purpose of establishing sustainable environments that involve local honey bee populations and ultimately how these ideas can be addressed through communication design. This literature review then informed the research methods to structure a coherent and meaningful design process that incorporates appropriate techniques. The combination of Research About Design and Research Through Design methods lead a Human-centered Design approach, with the integration of Communication Design principles aiming to direct the design output through the Communication practice: Design for the Education of the Public. The purpose and content of this design is then reinforced and directed through stakeholder perspectives and design suggestions. This section intends to combine opinions and concerns that are consistent across the majority of interviewees so that hierarchies of information can be formulated and matched to the purpose of using Communication Design. The sorting of information then justifies and elaborates on the literature collected in the first section and informs the types of content and visuals that can be used to address current barriers to honey bee population growth. The integration of a design criteria aims to target the main Communication Design principles, which considers the overall graphic, content and interactive qualities of the information being produced. Consequently, the final design prototype is formed by combining each section's findings, with the intent of the design relating to raising awareness and encouraging empathic relationships with our honey bee populations. The essential research goal is to aid in encouraging positive environmental actions within Wellington communities, and to start a conversation about the future condition of our local ecosystems and species.

\section{Future Pathways}

Future pathways for this outcome could include displaying the content across different media platforms, as the visuals provide a flexible approach to various issues concerning honey bee populations both locally and globally. The addition of more stakeholders could broaden the scope and understanding of organisational roles in Wellington, potential participants could include: local honey producers, restaurants and services that include bee products and practices, more professional beekeepers, Local Councils and the Wellington City Council. Different activities of the bee could also be targeted e.g. images within the beehive showing an internal insight that is not commonly seen by the public. Various types of photographic approaches could be explored e.g. filming, time lapse, scale and perspective techniques to encourage and develop photographics processes and therefore different insights of the bee. The relation to context could consider other local or global ecosystems including bee populations and how the relationship with surrounding environments and habitats enables or hinders bee productivity. The application of various Communication Design practices can be explored in terms of different medias, issues and messages wanting to be publicised. The aim of raising awareness in this thesis could be applied to future contexts through the application of other communication designs, with the content and visuals supporting current concerns in the honey bee industry. The main Communication Design practice of Design for the Education of the Public could consider other sustainable issues, using compelling visuals/ photographs to persuade and inform public actions and behaviours. The impactful nature of Communication Design should be intensified and used to address relevant social and cultural issues that can be normalised or universalised through design. 


\section{Personal Reflection}

Throughout this thesis my interpretation and appreciation for New Zealand's honey bee species has changed drastically. I have been continuously immersed in expert and industry knowledge, along with discovering new ways of impactful and purposeful design. My visual way of thinking and processing has lead to an exploration into my photographic capabilities and has extended my understanding of capturing meaningful ideas in a particular context. The redefinition of the current global issue of honey bee decline and the recontextualising of this large scale within a smaller Wellington context has been a challenge. This has reinvented appropriate sources and stakeholders to be involved to dictate the design content, through an experiential, holistic view of Wellington's bee industry. An insight into the positive attitudes and interests of people and organisations involved in this industry reflects a unique outlook and a healthy concern for the future sustainability of our environments. I believe that substantial involvement and interests of younger generations is needed to encourage conservation and to trigger an awareness of the potential future environmental realities we may face, specifically in New Zealand. My own sustainable behaviours and actions have changed for the better, as I can now appreciate the processes within nature that need protecting, and ecosystems that are vital to the overall health of our environments. In-depth research throughout this thesis has affected my perception of our native land, flora and fauna and has subsequently injected a willingness to practice positive environmental behaviours. As this environmental awareness will effect me for the rest of my life, I will be eternally grateful for this opportunity to study such an engaging and relevant topic. A particular passion for studying honey bees has accentuated my beliefs in their magnificent existence and the significant role they play in New Zealand's environmental, social and economic sectors. Following the legacy and passion of beekeepers in my family, new design ideas and outputs have connected me to past beliefs and practices and has shown me how honey bee species will continue to be vital for many years to come. 


\section{Reference List}

100 Resilient Cities. (2017). Urban Resilience. Retrieved from http://www.100resilientcities.org/ resilience\#/-_

Barr, S. (2003). Strategies for sustainability: Citizens and responsible environmental behaviour. Area, 35(3), 227-240.

Bartel, C., \& Garud, R. (2009). The role of narratives in sustaining organizational innovation. Organization Science 20(1), 107-117.

Beard, C. (2015). Honeybees (Apis mellifera) on public conservation lands: a risk analysis. Department of Conservation, Wellington. $21 \mathrm{p}$.

Bolchini, D., Yang, T., \& Garzotto, F. (2009, October). Evaluating the communication design of branded websites: a value-based framework. In Proceedings of the 27th ACM international conference on Design of communication (pp. 73-80). ACM.

Brown, M. (2016). Futurestorative : Working towards a new sustainability / Martin Brown.

Burgess, J., Harrison, C. M., \& Filius, P. (1998). Environmental communication and the cultural politics of environmental citizenship. Environment and planning A, 30(8), 1445-1460.

Choukeir, J. (2011). Defining Communication Design. Retrieved May 18, 2017, from http:// joannachoukeir.com/Defining-Communication-Design\#.WR0Vo9wIFaR

Chalmer, S. (2016). "The Hive" Sound and light installation controlled by bees. Retrieved from http://edition.cnn.com/2016/06/20/arts/the-hive-wolfgang-buttress/

Creswell, J. (2014). Research design : Qualitative, quantitative, and mixed methods approaches / John W. Creswell. (4th ed.). Thousand Oaks: SAGE Publications.

Federated Farmers of New Zealand (Inc.). (1966). from An Encyclopaedia of New Zealand, edited by A. H. McLintock. Te Ara - the Encyclopedia of New Zealand. Retreived from: http://www.TeAra. govt.nz/en/1966/farmers-organisations

Federated Farmers. (2016). NZ National Policy - Bee Issues. Retrieved from: http://www.fedfarm. org.nz/advocacy/National-Policy/Bees-Issues.asp

For the Love of Bees. (2017, March 6). Campaign To Make Auckland The Bee Capital of The World. Retrieved from https://www.fortheloveofbees.co.nz/news-1/2017/3/6/campaign-to-makeauckland-the-bee-capital-of-the-world

Frascara, J. (1997). User-Centered Graphic Design; Mass Communications and Social Change. London \& Bristol PA: Taylor \& Francis Ltd.

Frascara, J. (2004). Communication Design: Principles, Methods, and Practice. New York: Allworth Press.

Freeman, C., Robertson, A.B., Achilladelis, B.G., \& Jervis, P. (1972). Success and failure in industrial innovation

(Report on Project SAPPHO by the Science Policy Research Unit). London: University of Sussex, Center for the Study of Industrial Innovation.

Gillingham, A. (2012). 'Beekeeping'. Te Ara-The Encyclopedia of New Zealand. Updated 9 Nov 2012. www.teara.govt.nz/en/beekeeping (accessed December 2016). 
Goddard, M. A., Dougill, A. J., \& Benton, T. G. (2010). Scaling up from gardens: biodiversity conservation in urban environments. Trends in Ecology \& Evolution. 25(2), 90-98. http://doi. org/10.1016/j.tree.2009.07.016

Goodwin, M.; Scarrow, S.; Taylor, M. (2006). Supply of and demand for pollination hives in New Zealand. A briefing paper prepared for the Strategic Pollination Group. Ministry of Agriculture and Fisheries, New Zealand. 28p

Grainger, Mao, \& Buytaert. (2016). Environmental data visualisation for non-scientific contexts: Literature review and design framework. Environmental Modelling and Software, 85, 299-318.

Grimm, N. B., Faeth, S. H., Golubiewski, N. E., Redman, C. L., Wu, J., Bai, X., \& Briggs, J. M. (2008). Global change and the ecology of cities. Science, 319(5864), 756-760.

Guest, G., MacQueen, K. M. \& Namey, E. E. (2012). Applied thematic analysis. Thousand Oaks, CA: SAGE Publications Ltd. DOI: 10.4135/9781483384436

Haraway, D., \& Ebrary, Inc. (2008). When species meet / Donna J. Haraway. (Posthumanities ; 3). Minneapolis: University of Minnesota Press.

Honey Factory is "micro architecture" for bees. (2015, July 1). Dezeen. Retrieved September 7 , 2016, from http://www. dezeen.com/2015/07/01/francesco-faccin-honey-factory-urban-beehivebees-triennale-museum-milan-expo-2015/

Howlett, B.G. \& Donovan, B.J. (2012). A review of New Zealand's deliberately introduced bee fauna: current status and potential impacts. The New Zealand Entomologist. 33(1), 92. http://doi. org/10.1080/00779962.2010.9722196

Hutching, G. (2016, January 13). Manuka honey reaps rewards in overseas markets. Stuff. Retrieved from http://www.stuff.co.nz/business/farming/75829997/Manuka-honey-reaps-rewardsin-overseas-markets

Jacobson, R. E. (1999). Information design / edited by Robert Jacobson. Cambridge, Mass.: MIT Press.

Katz, J., \& Ebrary, Inc. (2012). Designing information : Human factors and common sense in information design / Joel Katz. Hoboken, New Jersey: Wiley.

Kew Royal Botanic Gardens. (n.d.). The Hive. Retrieved April 25, 2017, from http://www.kew.org/ kew-gardens/attractions/the-hive

Kirk, A. (2016). Data visualisation: a handbook for data driven design. SAGE

Kleinsmann, M., Maier, A., Roschuni, C., Goodman, E., \& Agogino, A. M. (2013). Communicating actionable user research for human-centered design. Artificial Intelligence for Engineering Design, Analysis and Manufacturing : AI EDAM, 27(2), 143-154. doi:http://dx.doi.org/10.1017/ S0890060413000048

Laurel, B. (2003). Design research : Methods and perspectives / Brenda Laurel. Cambridge, Mass.: MIT Press.

Lundquist, C. J., Ramsay, D., Bell, R., Swales, A., \& Kerr, S. (2011). Predicted impacts of climate change on new zealand's biodiversity. Pacific Conservation Biology, 17(3), 179-191. Retrieved from http://search.proquest.com/docview/921223184?accountid=14782 
Marsh, P. (1983). Messages that work : A guide to communication design / Patrick O. Marsh. Englewood Cliffs, N.J.: Educational Technology Publications. (Google Books)

Mäkelä, M. (2006). The art of research : Research practices in art and design / edited by Maarit Mäkelä \& Sara Routarinne. Helsinki: University of Art and Design Helsinki.

Moore, L., \& Kosut, M. (2013). Being with Bees: Intimate Engagements between Humans and Insects. In Buzz: Urban Beekeeping and the Power of the Bee. (pp. 85-122). NYU Press. Retrieved from http://www.jstor.org/stable/j.ctt9qfrd1.7

Muratovski, G. (2016). Research for designers. S.I.: Sage Publications.

Nature Study | Exhibition | Bartley and Company Art. (2015). Retrieved from http://www. bartleyandcompanyart.co.nz/exhibition,Anne_Noble, 276

Nemeth, C. (2004). Human factors methods for design : Making systems human-centered / Christopher P. Nemeth. Boca Raton, Fla. ; London: CRC Press.

Newman, C., \& Lieu, D.K. (2005). Comprehension scaffolding for multimedia: structuring technical animations for learning. ASEE/EDGD Proc. Mid-Year Meeting.

No Vertical Song | Exhibition | Two Rooms. (2015). Retrieved from http://tworooms.co.nz/ exhibition/anne-noble/

Pasley, J. (2017, March 7). Campaign to make Auckland the world's most liveable place for bees. Stuff. Retrieved from http://www.stuff.co.nz/environment/90088950/campaign-to-make-aucklandthe-worlds-most-liveable-place-for-bees

Raven, M.E., \& Flanders, A. (1996). Using contextual inquiry to learn about your audiences. SIGDOC Asterisk Journal of Computer Documentation 20(1), 1-13.

Rockefeller Foundation. (2017). 100 Resilient Cities. Retrieved from https://www. rockefellerfoundation.org/our-work/initiatives/100-resilient-cities/

Rouse, W. (2007). People and organizations : Explorations of human-centered design / William B. Rouse. Hoboken, N.J.: John Wiley.

Ryan, G., \& Bernard, H. (2003). Techniques to identify themes. Field Methods, 15(1),85-109. Retrieved from: http://dx.doi/10.1177/1525822X02239569

Sachs, J. D. (2012). From millennium development goals to sustainable development goals. The Lancet, 379(9832), 2206-2211.

Smuts-Kennedy, S. \& Taiaroa, T. (2014). The Park — POP Projects. Retrieved from http://www. pop.org.nz/projects/thepark

Steg, \& Vlek. (2009). Encouraging pro-environmental behaviour: An integrative review and research agenda. Journal of Environmental Psychology, 29(3), 309-317.

United Nations. (2015). The General Assembly. Transforming our world: the 2030 Agenda for Sustainable Development. Retrieved from: http://www.un.org/ga/search/view_doc.asp?symbol=A/ RES/70/1\&Lang=E

Visser, F. S. (2009). Bringing the everyday life of people into design. PhD Thesis. Delft University of Technology.

Vitousek, P. (1997). Human domination of Earth's ecosystems. Science., 277(5325), 494.

Wellington City Council. (2016). Towards 2040: Smart Capital. Retrieved August 10, 2016, from http://wellington.govt.nz/your-council/structure-and-vision/vision-2040/towards-2040-smart-capital

Wong, B., Yuen, T., Yuen, Belinda, Singapore Institute of Planners Content Provider, \& Wong, TaiChee. (2011). Eco-city Planning Policies, Practice and Design. 


\section{Appendix}

Ethics Approval Number 0000023700

Inclusive of participant information sheets, participant consent forms and interview questions. 
